\title{
Parametrix for a Degenerate Parabolic Equation and its Application to the Asymptotic Behavior of Spectral Functions for Stationary Problems
}

By

\author{
Chisato IWASAKI* and Nobuhisa IWASAKI**
}

\section{Contents}

Chapter 0. Introduction

$\S 0.1$. Simple Notations and Assumptions ...................... 580

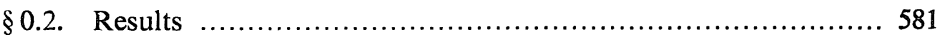

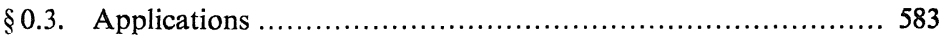

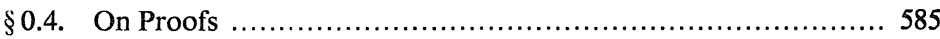

Chapter 1. Construction of a Parametrix in Terms of Pseudodifferential

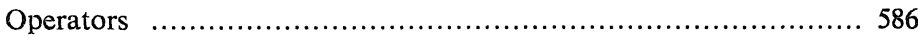

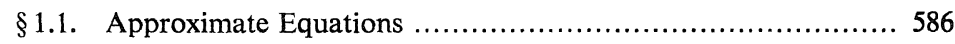

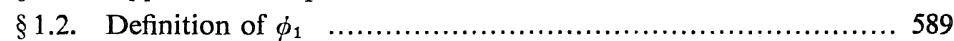

$\S 1.3$. A Class of Pseudodifferential Operators ....................... 595

$\S 1.4$. First Approximate Equation ................................. 599

$\S 1.5$. Solution of Second Approximate Equation $\ldots \ldots \ldots \ldots \ldots \ldots . \ldots 62$

$\S 1.6$. Induction and Estimates of the Parametrix ...................6 605

Chapter 2. Representation of the Fundamental Solution by Pseudodifferential

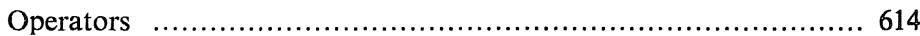

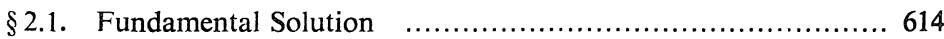

$\S 2.2$. An Application. Melin's Result (Gârding Type Inequality) ... 616

Chapter 3. Ambiguities of Complex Phase Functions $\ldots \ldots \ldots \ldots \ldots \ldots \ldots \ldots \ldots . \ldots 17$

$\S 3.1$. Replacement of Complex Phase Functions ...................6 618

$\S 3.2$. Special Case (Exact Double Characteristic) .................. 622

Chapter 4. Asymptotic Behavior of Trace ................................... 624

\$4.1. Assumptions and Conclusions ...........................6 624

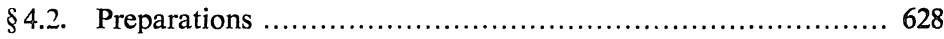

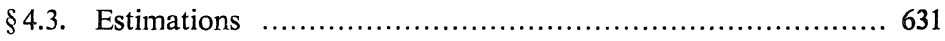

$\S 4.4$. Reconsideration about General Cases ........................ 638

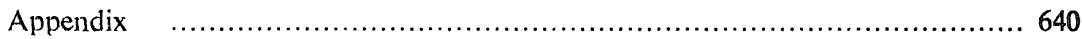

§A.1. Hamilton (Fundamental) Matrix ......................... 640

$\S A .2 . \quad$ Pseudodifferential Operators ................................ 644

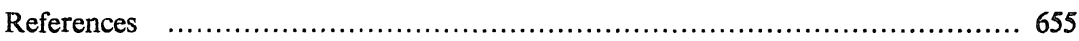

Received August 19, 1980.

* Department of Mathematics, Osaka University, Toyonaka, 560, Japan.

** Research Institute for Mathematical Sciences, Kyoto University. Kyoto 606, Japan. 


\section{Chapter 0. Introduction}

This paper is a detailed version of one announced in C. Iwasaki and N. Iwasaki [8]. (And also refer to [9].) We study a fundamental solution for an evolution equation

$$
\begin{aligned}
& ((\partial / \partial t)+p(x, D)) E(t)=0, \quad t>0, \\
& E(0)=I .
\end{aligned}
$$

$P$ is a classical pseudodifferential operator of order $m$, having an asymptotic expansion of the symbol $p(x, \xi)$ such that

$$
p(x, \xi) \sim p_{m}(x, \xi)+p_{m-1}(x, \xi)+p_{m-2}(x, \xi)+\cdots,
$$

where $p_{j}(x, \xi)$ is positively homogeneous of order $j$.

Usually this equation is called parabolic if the principal symbol $p_{m}$ of $P$ is positive $(\xi \neq 0)$ and the order $m$ is greater than 1 . In this case $P$ is strongly elliptic and satisfies a Gårding inequality (0.0.3) and an a-priori estimate (0.0.4).

$$
\begin{gathered}
\operatorname{Re}(p(x, D) u, u) \geqq \varepsilon\|u\|_{m / 2}^{2}-C\|u\|_{0}^{2}, \quad \text { for } u \text { of } \mathscr{S}\left(\mathbf{R}^{n}\right) . \\
\|u\|_{m+s}^{2} \leqq C_{s}\left(\|P u\|_{s}^{2}+\|u\|_{s}^{2}\right), \quad \text { for } u \text { of } \mathscr{S}\left(\mathbf{R}^{n}\right) .
\end{gathered}
$$

Therefore on a suitable function space the existence of fundamental solution $E(t)$ is shown by the theory of one parameter semigroups. On the other hand it is also shown in a constructive way by means of symbol calculations of pseudodifferential operators. Namely, a parametrix

$$
\begin{aligned}
& \sigma(E(t)) \sim f(t, x, \xi) \exp \left(-p_{m}(x, \xi) t\right), \\
& f=1+f_{1}+f_{2} \cdots,\left(f_{j} \exp \left(-p_{m} t\right) \text { belongs to } S_{1,0}^{-j}\right),
\end{aligned}
$$

is constructed and a fundamental solution is represented in terms of pseudodifferential operators with a parameter $t$ by using neither $(0.0 .3)$ nor $(0.0 .4)$. (Refer to C. Iwasaki [7].) It also follows that $E(t)$ belongs to $S^{-\infty}$ for any positive $t$. That is one of characters of parabolic types. Moreover a Gårding inequality and an a-priori estimate can be conversely proved by the constructed one.

Here we consider a more general case. Since the evolution equation should be well posed in some sense, we assume

$$
p_{m}(x, \xi) \geqq 0 \text { and } m>1 \text {. }
$$

$P(x, D)$ is not always elliptic because the principal symbol $p_{m}(x, \xi)$ may vanish 
somewhere $(\xi \neq 0)$. It seems natural in order to preserve properties of parabolic types that we assume hypoellipticity to $p(x, D)$ instead of ellipticity. We shall here call them degenerate parabolic types.

There are two related results. A. Melin [10] proves that the following $(0.0 .7)$ and $(0.0 .8)$ are equivalent if $p(x, \xi)$ satisfies $(0.0 .6)$.

(0.0.7) The subprincipal symbol plus $2^{-1}$ positive trace of fundamental matrix for $p_{m}(x, \xi)$ is positive on the characteristic set $\Sigma$ of $p_{m}$.

$$
\operatorname{Re}(p(x, D) u, u) \geqq \varepsilon\|u\|_{(m-1) / 2}^{2}-C\|u\|_{n}^{2}
$$

for some positive $\varepsilon$ and $C$, and for any $u$ of $C_{0}^{+\infty}(K),(K$ is a compact set).

According to parts of results by L. Hörmander [5], if $p(x, \breve{\zeta})$ satisfies $(0.0 .6)$ and (0.0.7), it holds (0.0.9) and so (0.0.10).

$$
\|u\|_{m-1+s}^{2} \leqq C_{s}\left(\|P u\|_{s}^{2}+\|u\|_{s}^{2}\right), \quad \text { for } u \text { of } C_{0}^{+\infty}(K) \text {. }
$$

$$
P \text { is hypoelliptic. }
$$

Therefore if $(0.0 .6)$ and $(0.0 .7)$ are assumed for $p(x, D)$, the existence of fundamental solution is shown by the theory of one parameter semigroup as well as in case of parabolic types.

One of next steps will be to know further informations about $E(t)$. For example "Is it a pseudodifferential operator?" According to R. Beals [1] a parametrix of $P$ is constructed if it satisfies (0.0.6) and (0.0.9). And B. Helffer has noted in [4] that the fundamental solution $E(t)$ belongs to $S_{1 / 2,1 / 2}^{0}$ if $(0.0 .8)$ and a result* in R. Beals [1] hold. (*If $P$ belongs to $S^{0}$ and if $P$ is an isomorphism on $\mathbb{L}^{2}$, then the inverse $P^{-1}$ also belongs to $S^{0}$.) However the form of symbol is not clear. Meanwhile A. Menikoff and J. Sjöstrand [12] has constructed a parametrix of form $f \exp \phi$ in terms of Fourier integral operators with complex phase functions (refer to A. Melin and J. Sjöstrand [11]) under (0.0.6), (0.0.7) and the restriction that $p_{m}$ vanishes exactly double on the characteristic set $\Sigma$ of $p_{m}$ and that $\Sigma$ is symplectic manifold, though L. Boutet de Monvel, A. Grigis and B. Helffer [2] had constructed a parametrix for $P$ if it had been only got to be a pseudodifferential operator. Consequently they have calculated the rate of $\operatorname{Tr} E(t)$ as $t$ tends to zero. They have proved it using $(0.0 .8)$ and have not said positively that their parametrix was a pseudodifferential operator.

In this paper under $(0.0 .6)$ and $(0.0 .7)$ we shall prove that $E(t)$ is a pseudodif- 
ferential operator of $S_{1 / 2,1 / 2}^{0}$ with a parameter $t$ belonging to $S^{-\infty}$ if $t>0$, and has a parametrix of pseudodifferential operators with symbols of form $f \exp \phi$ ( $\phi$ is real valued if the subprincipal symbol is real.) Here $\phi$ and $f$ will be obtained by means of symbol calculations of $p$. Especially the leading term will be given explicitly. The inequalities (0.0.8) and (0.0.9) will follow as corollaries. The same results about the trace of $E(t)$ as A. Menikoff and J. Sjöstrand got will be proved without the condition that $\Sigma$ is symplectic. (They have also extended their result to this case in [13] and to the general case in [14].) And the conditions will be weakened further in some part.

Remarks. 1) The left hand side in (0.0.7) must be non negative if (0.0.1) is well posed in some global sense.

2) When we studied these problems, the Weyl symbol for pseudodifferential operators was very useful for us. From now on we shall use only it as symbol representations of pseudodifferential operators instead of the usual one. (Refer to Appendix.)

\section{§0.1. Simple Notations and Assumptions}

We employ the Weyl symbol for pseudodifferential operators, that is, a symbol $a(x, \xi)$ defines an operator $a(x, D)$ by

$$
a(x, D) u=(2 \pi)^{-n} \int_{\Omega} e^{i(x-y) \xi} a((x+y) / 2, \xi) u(y) d y d \xi \text { for } u \text { of } C_{0}^{+\infty}
$$

where $\Omega=\mathbf{R}^{n} \times \mathbf{R}^{n}$. Hence $p_{m-1}$ is the subprincipal symbol of $P$ in usual sence. In fact the relation between a Weyl symbol $a(x, \xi)$ and an usual one $b(x, \xi)$ of $S_{\rho, \delta}^{m}(0 \leqq \delta<\rho \leqq 1)$ is given by

$$
a(x, \breve{\zeta}) \equiv \exp \left\{-(2 i)^{-1} \sum \partial_{x} \partial_{\xi}\right\} b(x, \xi) \bmod S^{-\infty} .
$$

$\nabla^{k} a$ stands for a section of $T^{* k}\left(T^{*} \mathbf{R}^{n}\right), k$-th symmetric tensor of $T^{*}\left(T^{*} \mathbf{R}^{n}\right)$, defined by (0.1.2) with respect to the canonical coordinate of $T^{*} \mathbf{R}^{n}$.

(0.1.2) $\quad \sum_{|\alpha+\beta|=k} C_{\alpha \beta}^{k} a_{(\beta)}^{(\alpha)}(d \xi)^{\alpha}(d x)^{\beta}, C_{\alpha \beta}^{k}=k ! / \alpha ! \beta ! \quad$ and $\quad a_{(\beta)}^{(\alpha)}=\partial_{\xi}^{\alpha} \partial_{x}^{\beta} a(x, \xi)$.

A linear map defined by $\nabla^{k} a$ from $T^{j}\left(T^{*} \mathbf{R}^{n}\right)$ to $T^{* k-j}\left(T^{*} \mathbf{R}^{n}\right)$ is denoted by the same notation $\nabla^{k} a$. $\sigma^{1}$ is the canonical two form $d \xi \wedge d x=\sum d \xi^{j} \wedge d x_{j}$ on $T^{*} \mathbf{R}^{n}$. For the principal symbol $p_{m}$ the Hamilton vector field $h$ is defined by $\sigma^{1}(u, h)=\nabla p_{m}(u)$ and the Hamilton (fundamental) matrix $\mathscr{F}$ by $\sigma^{1}(u, \mathscr{F} v$ ) $=Q(u, v), Q(u, v)=\left\langle u, \nabla^{2} p_{m} v\right\rangle$. If we define $J_{1}$ by $\sigma^{1}\left(u, J_{1} f\right)=f(u)$, then 
$h=J_{1} \nabla p_{m}$ and $\mathscr{F}=J_{1} \nabla^{2} p_{m}$. We put $A=i \mathscr{F}$ and $b=i h$. $\quad \operatorname{Tr}^{\sim} A$ stands for the sum of real parts of eigenvalues of $A$ which are positive. On the characteristic set $\Sigma$ of $p_{m}, \nabla^{2} p_{m} \geqq 0$ if $p_{m} \geqq 0$. This implies that $A$ has only real eigenvalues. $\operatorname{Tr} \sim A$ is the positive trace of $A$, that is, the sum of positive eigenvalues.

Remark. Here wc also call $A$ and $b$ Hamilton matrix and vector, respectively, because they are corresponded to a complex Hamiltonian as $\mathscr{F}$ and $h$ are to a real Hamiltonian.

Remark. Definitions of $\nabla^{k} a$, the Hamilton vector field and matrix will be modified by a weight function for the simplicity of calculations in proofs. But $\operatorname{Tr} \sim A$ and any function of $A$ and $b$ appearing in conclusions are free of such a weight function. (Refer to Chapter 1.)

Throughout this paper we assume the following (0.1.3).

\section{Condition (A).}

$$
p_{m} \geqq 0 \quad \text { on } \quad T^{*} \mathbb{R}^{n} \quad \text { and } \quad 2 \operatorname{Re} p_{m-1}+\operatorname{Tr}^{\sim} A \geqq c|\xi|^{n-1}
$$

on the characteristic set $\Sigma=\left\{p_{m}=0\right\}$ for a positive constant $c$.

\section{§0.2. Results}

Theorem 0.1. Under Condition $(A)$ a fundamental solution $E(t)$ of $(0.0 .1)$ is constructed as a pseudodifferential operator with a symbol belonging to $L_{0}^{0}$. $E(t)$ belongs to $S^{-\infty}$ if $t$ is positive. Moreover $E(t)$ has the following asymptotic expansion.

$$
\begin{aligned}
& E(t)=\sum_{j=0}^{N} f_{j} \exp \phi+g_{N}, \\
& f_{0} \equiv 1, f_{j} \exp \phi \quad \text { belongs to } L_{0}^{-\varepsilon j} \text { and } \\
& g_{N} \text { belongs to } L_{0}^{-\varepsilon(N+1)},(0<\varepsilon<1 / 6) .
\end{aligned}
$$

Here the function $\phi$ is defined $b \mathrm{y}^{\cdot}(0.2 .3-8)$. At a neighborhood of $\Sigma \times\{t=0\}$

$$
\begin{aligned}
\phi_{1}= & -p_{m} t-p_{m-1} t-\sigma^{1}(b t / 2, F(A t / 2) b t / 2) \\
& -2^{-1} \operatorname{Tr}(\log [\cosh (A t / 2)]),
\end{aligned}
$$

and otherwise

$$
\phi_{2}=-p_{m} 1-\langle\xi\rangle^{m-1} t,
$$


namely,

$$
\phi=\psi_{1} \phi_{1}+\left(1-\psi_{1}\right) \phi_{2},
$$

where

$$
\begin{aligned}
& \psi_{k}=\psi_{k}^{1} \psi_{k}^{2}, \quad(k=1,2) \\
& \psi_{k}^{1}=\psi\left(k^{-1} p_{m}\langle\xi\rangle^{1-m-2 \varepsilon}\right), \\
& \psi_{k}^{2}=\psi\left(k^{-1} t\langle\xi\rangle^{m-1-\delta}\right),
\end{aligned}
$$

and

$\psi(s)$ is a function of $C^{+\infty}[0,+\infty)$ such that $\psi=1(s \leqq 1), \psi=0(s \geqq 2)$, $\psi^{\prime}<0(1<s<2)$ and $\left|\psi^{(n)}\right| \leqq c_{n \tau}(1-\psi)^{\tau}$ if $0<\tau<1$.

The relation between $\delta$ and $\varepsilon$ is

$$
0<12 \delta<1-6 \varepsilon<1 \text {. }
$$

Remark. We use a notation $L_{\rho}^{m}$, for a class of pseudodifferential operators, which is equal to $S_{1 / 2+\rho, 1 / 2-\rho}^{m}$ of Hörmander's class.

Remark. The condition (0.2.8) guarantees that $F(A t / 2), \cosh (A t / 2)$ and so on are well defined and that $\exp \phi$ belongs to $L_{0}^{0}$.

Remark. Refer to Section 1.5 for the way of construction of $f_{j}$, which are functions of $p$ and its derivatives.

Since $\int_{0}^{c} E(t) d t(c>0)$ is a parametrix of $P$, we obtain the followings.

Corollary [A. Melin and L. Hörmander]. There exist constants $\lambda$ and $C_{\text {s }}$ such that for any $u$ of $\mathscr{S}\left(\mathbf{R}^{n}\right)$

$$
\operatorname{Re}((P+\lambda) u, u) \geqq 0
$$

and

$$
\|u\|_{m-1+s}^{2} \leqq C_{s}\left(\|P u\|_{s}^{2}+\|u\|_{s}^{2}\right) .
$$

Remark. The expression may be a little different from A. Melin's result but it is essentially same.

Example. We consider on $\mathbf{R}^{2 n+l}$

$$
P=\sum_{j=1}^{k}\left(D_{x_{j}}^{2}+x_{j}^{2} D_{y_{j}}^{2}\right)+\sum_{j=1}^{l} D_{z_{j}}^{2} .
$$

Then the symbol of $E(t)$ is given by 


$$
\begin{aligned}
& \prod_{j=1}^{k}\left\{\cosh \left|\eta_{j}\right| t\right\}^{-1} \\
& \quad \times \exp \left\{-\sum_{j=1}^{k}\left(\xi_{j}^{2}+x_{j}^{2} \eta_{j}^{2}\right)\left|\eta_{j}\right|^{-1} \tanh \left(\left|\eta_{j}\right| t\right)-\sum_{j=1}^{l} \zeta_{j}^{2} t\right\} \\
& =\exp \phi_{1},
\end{aligned}
$$

where $\Sigma=\left\{\xi_{j}=0, x_{j} \eta_{j}=0(1 \leqq j \leqq k), \check{\zeta}_{i}=0(1 \leqq i \leqq l)\right\}$.

We consider more restrictive cases, that claim $\Sigma$ to be exactly double.

Condition (B). The principal symbol $p_{m}$ vanishes exactly to second order on the characteristic set $\Sigma$, that is, $p_{m}(X) \geqq c(X) d(X, \Sigma)^{2},(|\xi|=1, X=(x, \xi)$ and $c(X)>0)$.

Remark. $d(X, \Sigma)$ is the distance of $X$ to $\Sigma$ with respect to the metric of $\mathbf{R}^{n} \times \mathbf{R}_{+} \times S^{n-1}$, that is,

$$
d((x, \xi),(y, \eta))=\left\{|x-y|^{2}+(|\xi|-|\eta|)^{2}+|\xi /| \xi|-\eta /| \eta||^{2}\right\}^{1 / 2} .
$$

Remark. In this case $\Sigma$ is necessarily an infinitely differentiable submanifold of $T^{*} \mathbf{R}^{n} \backslash\{0\}$. Therefore $d(X, \Sigma)$ is an infinitely differentiable function at a conic neighborhood of $\Sigma$ and there exists an infinitely differentiable mapping $a(X)$ valued in $\Sigma$ such that $d(X, a(X))=d(X, \Sigma)$.

Theorem 0.2. Under Conditions $(A)$ and $(B)$ the phase function $\phi_{1}$ at a neighborhood of $\Sigma$ can be replaced with $\phi_{3}$ defined by (0.2.9) if we add a condition that $8 \varepsilon \leqq 1$. In fact Theorem 0.1 is valid for the same $\varepsilon$ on any compact set of $\mathbb{R}^{n}$.

$$
\begin{aligned}
\phi_{3}= & -p_{m-1}(a) t+i \sigma^{1}((a-X), \tanh (A(a) t / 2)(a-X)) \\
& -2^{-1} \operatorname{Tr}(\log [\cosh (A(a) t / 2)]),
\end{aligned}
$$

where $a=a(X)$ is an infinitely differentiable mapping from a neighborhood of $\Sigma$ to $\Sigma$ such that $|d(X, a(X))-d(X, \Sigma)| \leqq c d(X, \Sigma)^{2}$.

\section{§0.3. Applications}

We can calculate $\operatorname{Tr} E(t)$ as $t$ tends to zero, using Theorems 0.1 and 0.2. Applying Karamata's Tauberian Theorem to it, the asymptotic behavior of spectral function is obtained.

Let $M$ be an infinitely differentiable compact manifold and $d M$ be a positive smooth density on it. We assume Condition (C) through out this section.

Condition $(\mathbb{C}) . \quad P$ is a formally selfadjoint pseudodifferential operator on $M$, that is, 


$$
\int_{M} P u v d M=\int_{M} u P v d M, \text { for any } u \text { and } v \text { of } C^{+\infty}(M) \text {. }
$$

Theorem 0.3. Under Condition $(A)$ and $(C)$

$$
\operatorname{Tr} E(t)=(1+o(1))(2 \pi)^{-n} \int_{T^{*} M} \exp \phi d x d \xi,
$$

as $t$ tends to zero, where $d x d \xi$ is the Liouville density on $T^{*} M$.

Remark. In this theorem, the function $\langle\xi\rangle$ used in the definitions of $\phi_{2}$ (0.2.5) and of $\psi_{1}(0.2 .7)$ should be replaced to a positive symbol of elliptic operator of order 1 defined on $\boldsymbol{M}$.

We consider two more restrictive cases to get exact rates. One is Condition (B) and the other is the following Condition (D).

Condition (D). $\int_{\left\{p_{m} \leqq 1\right\}} d x d \xi<+\infty$.

Remark. Since the principal and subprincipal symbols are well defined on $T^{*} M$, Conditions (A), (B) and (D) are well defined to $P$.

Remark. Under Condition (B) the characteristic set $\Sigma$ is divided as $\Sigma$ $=\mathrm{V}_{\text {disjoint }} \Sigma^{j}\left(\Sigma^{j}\right.$ are connected components of $\left.\Sigma, j=1, \ldots, l\right)$. Codim $\Sigma$ is defined by

$$
d=\operatorname{codim} \Sigma=\min _{j}\left\{\operatorname{codim} \Sigma^{j}\right\} .
$$

We denote the union of $\Sigma^{j}$ having the codimension of just $d$ by $\Sigma^{0}$.

Theorem 0.4. Under Conditions $(A)$ and $(C)$

(1) $P$ with the domain $=C^{+\infty}(M)$ is a semi-bounded essentially selfadjoint operator on $\boldsymbol{L}^{2}(M, d M)$.

(2) $P$ has only discrete spectrum.

(3) Let $N(\lambda)$ be the number of eigenvalues which are less than $\lambda$.

Under Conditions $(A),(B)$ and $(C)$, as $\lambda$ tends to infinity,
(a) $N(\lambda)=\left\{C_{1}+o(1)\right\} \lambda^{n / m}$
if $n-m d / 2<0$,
(b) $N(\lambda)=\left\{C_{2}+o(1)\right\} \lambda^{n / m} \log \lambda$
if $n-m d / 2=0$,

and

(c) $N(\lambda)=\left\{C_{3}+o(1)\right\} \lambda^{(n-d / 2) /(m-1)}$ if $n-m d / 2>0$.

Under Conditions $(A),(D)$ and $(C)$, as $\lambda$ tends to infinity,

(d) $N(\lambda)=\left\{C_{1}+o(1)\right\} \lambda^{n / m}$.

Here $C_{j}$ are given by

$$
C_{1}=(2 \pi)^{-n} \Gamma(n / m+1)^{-1} \int_{T^{*} M} \exp \left(-p_{m}\right) d x d \xi,
$$




$$
\begin{aligned}
C_{2}= & m^{-1}(2 \pi)^{-(n-d / 2)} \Gamma(n / m+1)^{-1} \\
& \times \int_{\Sigma^{0}}\left(p_{m-1}+2^{-1} \operatorname{Tr}^{\sim} A\right) \exp \left(-p_{m-1}-2^{-1} \operatorname{Tr}^{\sim} A\right) d \Sigma^{0},
\end{aligned}
$$

and

$$
\begin{aligned}
C_{3}= & (2 \pi)^{-(n-d / 2)} \Gamma((n-d / 2) /(m-1)+1)^{-1} \\
& \times \int_{\Sigma^{0}}\left[\operatorname{det}\left\{(A / 2)^{-1} \sinh (A / 2)\right\}\right]^{-1 / 2} \exp \left(-p_{m-1}\right) d \Sigma^{0} .
\end{aligned}
$$

Remark. $d \Sigma^{0}$ is an induced density on $\Sigma^{0}$ by $p_{m}$ and $d x d \xi$. If $(u, v)$ is a local coordinate such that $\Sigma^{0}=\{u=0\}$ (locally), we define it as $d \Sigma^{0}=\left[\operatorname{det}\left(H_{u u}\right)\right]^{-1 / 2} \Phi d v$, where $\Phi d u d v=d x d \xi$ and $H_{u u}$ is the Hesse matrix of $p_{m}$ with respect to the variable $u$.

Remark. In the case that $n-m d / 2=0, p_{m-1}+2^{-1} \operatorname{Tr} \sim$ is changeable to any other positive function of homogeneous order $m-1 . \quad C_{2}$ depends only on $d \Sigma^{0}$. (Refer to (4.3.35).)

\section{$\S 0.4$. On Proofs}

If we assume that $\exp \phi$ belongs to $L_{0}^{0}$, we get (0.4.1) by applying the expansion formula of products of two pseudodifferential operators with Weyl symbols. (Refer to Chapter 1.)

$$
\begin{aligned}
& ((d / d t)+P) \circ \exp \phi \\
= & (d / d t) \exp \phi+\sum_{k=0}^{2}(2 i)^{-h}(k !)^{-1} \sigma_{k}\left(p_{m}, \exp \phi\right)+p_{m-1} \exp \phi, \\
& \bmod L_{0}^{m-1-1 / 2} .
\end{aligned}
$$

We shall find $\phi$ such that it will belong to $L_{0}^{m-1-\varepsilon}$. In fact $\phi$ defined in Theorem 0.1 satisfies it. Especially $\phi_{1}$ satisfies approximately (0.4.2) at a neighborhood of $\Sigma \times\{t=0\}$.

$$
\begin{array}{ll}
\text { (0.4.2) } & (d / d t) \phi_{1}+\sum_{k=0}^{2}(2 i)^{-k}(k !)^{-1} \sigma_{k}\left(p_{m}, \exp \phi_{1}\right) \exp \left(-\phi_{1}\right)+p_{m-1}=0 \\
& \left.\phi_{1}\right|_{t=0}=0 .
\end{array}
$$

Differentiating twice this equation, we get an approximate equation (0.4.3) for $X=i J_{1} \nabla^{2} \phi_{1}$.

$$
\begin{gathered}
(d / d t) X+A-4^{-1} A X^{2}=0 \\
\left.X\right|_{t=0}=0 .
\end{gathered}
$$

The solution of this equation is given by $X=-2 \tanh (A t / 2)$. Going back to 
(0.4.2) $\phi_{1}$ is obtained. Next the transport equations $(0.4 .4)$ will be solved approximately and $f=1+f_{1}+f_{2}+\cdots$ will be obtained.

$$
\begin{aligned}
& (d / d t) f+\sum_{k=1}^{2}(2 i)^{-k}(k !)^{-1}\left\{\sigma_{k}\left(p_{m}, f \exp \phi_{1}\right)-\sigma_{k}\left(p_{m}, \exp \phi_{1}\right) f\right\} \\
& \times \exp \left(-\phi_{1}\right)=h, \\
& \left.f\right|_{t=0}=0 .
\end{aligned}
$$

However $\tanh (A t / 2), F(A t / 2)$ and so on will appear in the expression of $\phi$ and $f_{j}$ obtained in this way. The detailed discussions are needed to show that they are well defined and that $f_{j} \exp \phi$ belongs to $L_{0}^{-\varepsilon j}$. We shall take the necessary steps in Chapter 1.

Once a parametrix has obtained, a fundamental solution $E(t)$ will be obtained by solving the Volterra's integral equation (0.4.5) of pseudodifferential operators, where $E_{N}(t)=\sum_{j=0}^{N} f_{j} \exp \phi$ and $G_{N}(t)=((d / d t)+P) E_{N}(t)$.

$$
E(t)+\int_{0}^{t} E(t-s) G_{N}(s) d s=E_{N}(t) .
$$

This part will be shown in Chapter 2. Theorem 0.2 will be proved in Chapter 3. Chapter 4 will be put to prove Theorem 0.3 and 0.4 . Some notes about Hamilton matrices and pseudodifferential operators will be given at Appendix.

\section{Chapter 1. Construction of a Parametrix in Terms of Pseudodifferential Operators}

This chapter is the main part of this paper. We prove Theorem 0.1 . In Section 1.1 we give two equations. One is approximately satisfied by the complex phase function given in (0.2.3) (Section 1.4), which is exactly constructed in Section 1.2 and the other is a transport equation with respect to the complex phase function. The amplitude functions satisfy it inductively (Section 1.5). The proof is completed in Section 1.6.

\section{§1.1. Approximate Equations}

We start with a proposition for the expansion formula of the product of two pseudodifferential operators.

Notation. Throughout this paper except for Introduction, $\nabla^{k} a$ means a 
weighted one, that is, for an infinitely differentiable function $a(x, \xi)$ it is defined by

$$
\begin{aligned}
& \nabla^{k} a=\sum_{|\alpha+\beta|=k} C_{\alpha \beta}^{k} a_{(\beta)}^{(\alpha)}(d \xi)^{\alpha}(d x)^{\beta}, \quad C_{\alpha \beta}^{k}=k ! / \alpha ! \beta ! \quad \text { and } \\
& a_{(\beta)}^{(\alpha)}=\langle\xi\rangle(|\alpha|-|\beta|) / 2 \partial_{\xi}^{\alpha} \partial_{x}^{\beta} a(x, \xi) .
\end{aligned}
$$

Therefore $A$ and $b$ are weighted according as the definition in Section 0.1.

Remark. If $p$ belongs to $L_{\rho}^{m}$, then $\nabla^{k} p$ belongs to $L_{\rho}^{m-\rho k}$.

Proposition 1.1. If $a_{i}$ belong to $S_{\rho(i) \delta(i)}^{m(i)}$ and $\rho(i)>\delta(3-i), i=1,2$, then the symbol $a_{1} \circ a_{2}$ of the product operator $a_{1}(x, D) a_{2}(x, D)$ has the asymptotic expansion.

$$
a_{1} \circ a_{2} \equiv \sum_{k=0}^{+\infty}(2 i)^{-k}(k !)^{-1} \sigma_{k}\left(\nabla^{k} a_{1}, \nabla^{k} a_{2}\right) \quad \bmod S^{-\infty} .
$$

Proof. It is given at Appendix.

$$
\text { q.e.d. }
$$

Remarks. 1) $\sigma_{k}$ are bilinear forms on $T^{* k}\left(T^{*} \mathbb{R}^{n}\right)$. $\left(T^{* 0}\left(T^{*} \mathbb{R}^{n}\right)\right.$ $\equiv T^{*} \mathbb{R}^{n} \times \mathbf{C}$.) $\sigma_{0}(u, v)=u v, \sigma_{1}(u, v)=\left\langle J_{1} u, v\right\rangle$ and $\sigma_{k}$ are natural extensions of $\sigma_{1}$ on $T^{* k}\left(T^{*} \mathbb{R}^{n}\right)$. (Refer to Appendix.)

2) It is a special feature of Weyl symbols that a well regulated symbol appears in each term of the expansion.

3) $\sigma_{k}\left(\nabla^{k} a_{1}, \nabla^{k} a_{2}\right)$ may be denoted by $\sigma\left(\nabla^{k} a_{1}, \nabla^{k} a_{2}\right)$ or $\sigma_{k}\left(a_{1}, a_{2}\right)$.

4) The $n$-th partial sum of (1.1.1), $\sum_{k=0}^{n}(2 i)^{-k}(k !)^{-1} \sigma_{k}\left(a_{1}, a_{2}\right)$, is denoted by $a_{1{ }^{\circ}(n)} a_{2}$.

Let us consider the product of $p$ and $\exp \phi$. We assume that $\exp \phi$ belongs to $L_{0}^{0} . p$ in (0.0.1) belongs to $L_{1 / 2}^{m}$. Therefore, $\sigma_{k}(p, \exp \phi)$ belongs to $L_{0}^{m-k / 2}$, because $\nabla^{k} p$ and $\nabla^{k}(\exp \phi)$ belong to $L_{1 / 2}^{m-k / 2}$ and $L_{0}^{0}$, respectively. So we get (1.1.2), where $g_{0} \exp \phi$ belongs to $L_{0}^{m-3 / 2}$.

$$
\begin{aligned}
& ((d / d t)+p) \circ \exp \phi \\
& \quad \equiv \phi_{t} \exp \phi+\sum_{k=0}^{2}(2 i)^{-k}(k !)^{-1} \sigma_{k}(p, \exp \phi)+g_{0} \exp \phi \quad \bmod S^{-\infty} .
\end{aligned}
$$

Outside of the characteristic set $\Sigma$ of $p_{m}, \sigma_{0}\left(p_{m}, \exp \phi\right)=p_{m} \exp \phi$ is the term with the heighest order $m$. It is natural that the equation satisfied by $\phi$ seems to be (1.1.3).

$$
\phi_{t} \exp \phi+p_{m} \exp \phi=0, \text { that is, } \phi_{t}+p_{m}=0 .
$$

On the other hand $p_{m}$ and $\nabla p_{m}$ vanish on $\Sigma$. $(2 i)^{-2} 2^{-1} \sigma_{2}\left(p_{m}\right.$, exp $\left.\phi\right)+p_{m-1} \exp \phi$ will be the term with the heighest order $m-1$ there. In fact it will be clear later that Condition (A) guarantees it. Therefore we think of (1.1.4) as the 
equation that the complex phase function $\phi$ should satisfy on a neighborhood of $\Sigma$.

$$
\phi_{t} \exp \phi+\sum_{k=0}^{2}(2 i)^{-k}(k !)^{-1} \sigma_{k}\left(p_{m}, \exp \phi\right)+p_{m-1} \exp \phi=0 .
$$

Calculating $\sigma_{k}\left(p_{m}, \exp \phi\right)$ we get the equation (1.1.5).

$$
\begin{aligned}
& \phi_{t}+p_{m}+p_{m-1}+(2 i)^{-1} \sigma_{1}\left(\nabla p_{m}, \nabla \phi\right)+(2 i)^{-2} 2^{-1} \sigma_{2}\left(\nabla^{2} p_{m}, \nabla \phi \nabla \phi\right) \\
& \quad+(2 i)^{-1} 2^{-1} \sigma_{2}\left(\nabla^{2} p_{m}, \nabla^{2} \phi\right)=0 .
\end{aligned}
$$

This is rewritten as follows. (Refer to Appendix.)

$$
\begin{aligned}
& \phi_{t}+p_{m}+p_{m-1}+(2 i)^{-1}\left\langle J_{1} \nabla p_{m}, \nabla \phi\right\rangle-(2 i)^{-2} 2^{-1}\left\langle\nabla \phi, J_{1} \nabla^{2} p_{m} J_{1} \nabla \phi\right\rangle \\
& \quad-(2 i)^{-2} 2^{-1} \operatorname{Tr}\left(J_{1} \nabla^{2} p_{m} J_{1} \nabla^{2} \phi\right)=0 .
\end{aligned}
$$

We call (1.1.4), also (1.1.5) and (1.1.6), the first approximate equation.

Remark. It is enough for the complex phase function $\phi$ to satisfy approximately (1.1.4), that is, to find $\phi$ such that

$$
\begin{aligned}
\phi_{t} & +p_{m}+p_{m-1}+(2 i)^{-1} \sigma_{1}\left(\nabla p_{m}, \nabla \phi\right) \\
& +(2 i)^{-2} 2^{-1} \sigma_{2}\left(\nabla^{2} p_{m}, \nabla \phi \nabla \phi+\nabla^{2} \phi\right)=g_{1},
\end{aligned}
$$

where $g_{1} \exp \phi$ belongs to $L_{0}^{m-1-\varepsilon}(\varepsilon>0)$.

Next we look for a transport equation in order to make the remainder term $\left(g_{0}+g_{1}\right) \exp \phi$ of (1.1.2) and (1.1.7) vanish inductively. Let the remainder term $g \exp \phi$ belong to $L_{0}^{l+m-1}$. We will find an amplitude function $a$ such that (1.1.8) the order of $\left(\partial_{t}+p\right) \circ a \exp \phi-g \exp \phi \quad$ is lower than $l+m-1$.

We assume that we could find $a$ such that $a \exp \phi$ belongs to $L_{0}^{l}$. Operating $\left(\partial_{t}+p\right)$ to $a \exp \phi$, we get (1.1.9).

$$
\begin{aligned}
& \left(\partial_{t}+p\right) \circ a \exp \phi \\
\equiv & \left(a_{t}+a \phi_{t}\right) \exp \phi+\sum_{k=0}^{+\infty}(2 i)^{-1}(k !)^{-1} \sigma_{k}(p, a \exp \phi) \quad \bmod S^{-\infty} .
\end{aligned}
$$

$\sigma_{k}\left(p_{m}, a \exp \phi\right)$ belongs to $L_{0}^{l+m-3 / 2}$ if $k \geqq 3$, and $\sigma_{k}\left(p-p_{m}, a \exp \phi\right)$ belongs to $L_{0}^{l+m-3 / 2}$ if $k \geqq 1$. These imply (1.1.10).

$$
\begin{aligned}
& \left(\partial_{t}+p\right) \circ a \exp \phi \\
& \equiv\left(a_{t}+a \phi_{t}\right) \exp \phi+\sum_{k=0}^{2}(2 i)^{-1}(k !)^{-1} \sigma_{k}\left(p_{m}, a \exp \phi\right) \\
& \quad+p_{m-1} a \exp \phi, \quad \bmod L_{0}^{l+m-3 / 2}, \\
& =\left[a_{t} \exp \phi+\sum_{k=1}^{2}(2 i)^{-1}(k !)^{-1}\left\{\sigma_{k}\left(p_{m}, a \exp \phi\right)-a \sigma_{k}\left(p_{m}, \exp \phi\right)\right\}\right] \\
& \quad+a\left\{\phi_{t} \exp \phi+\sum_{k=0}^{2}(2 i)^{-1}(k !)^{-1} \sigma_{k}\left(p_{m}, \exp \phi\right)+p_{m-1} \exp \phi\right\} .
\end{aligned}
$$


We can expect for the second term $a g_{1} \exp \phi$ to belong to $L_{0}^{l+m-1-\varepsilon},(\varepsilon>0)$ by (1.1.7). So we get (1.1.11).

(1.1.11) $\left(\partial_{t}+p\right) \circ a \exp \phi$

$$
\begin{aligned}
\equiv a_{t} \exp \phi+\sum_{k=1}^{2}(2 i)^{-1}(k !)^{-1}\left\{\sigma_{k}\left(p_{m}, a \exp \phi\right)-a \sigma_{k}\left(p_{m},\right.\right. & \exp \phi)\}, \\
& \bmod L_{0}^{l+m-1-\varepsilon} .
\end{aligned}
$$

This implies that (1.1.8) holds if we define $a$ by a solution of (1.1.12).

$$
\begin{aligned}
& a_{t} \exp \phi+\sum_{k=1}^{2}(2 i)^{-1}(k !)^{-1}\left\{\sigma_{k}\left(p_{m}, a \exp \phi\right)-a \sigma_{k}\left(p_{m}, \exp \phi\right)\right\} \\
& \quad=g \exp \phi .
\end{aligned}
$$

We can rewrite (1.1.12) as (1.1.13).

$$
\begin{aligned}
a_{t} & +(2 i)^{-1} \sigma_{1}\left(\nabla p_{m}, \nabla a\right)+(2 i)^{-1} 2^{-1} \sigma_{2}\left(\nabla^{2} p_{m}, \nabla a \nabla \phi+\nabla^{2} a\right) \\
& =g .
\end{aligned}
$$

We call (1.1.12) and (1.1.13) the second approximate equation.

Remark. It is also enough to get an approximate solution.

\section{§1.2. Definition of $\phi_{1}$}

$$
\begin{aligned}
\phi_{1}= & -p_{m} t-p_{m-1} t-\sigma^{1}(b t / 2, F(A t / 2) b t / 2) \\
& -2^{-1} \operatorname{Tr}(\log [\cosh (A t / 2)])
\end{aligned}
$$

We explain how to have found $\phi_{1}(0.2 .3)$ or (1.2.1) before we prove for $\phi_{1}$ to be well defined. If $\phi_{1}$ is a solution of the first approximate equation (1.2.2) the derivatives of the both side of its equation also have to hold. In them we neglect the terms which include the derivatives of $\phi_{1}$ and $p_{m}$ with more than second order by the same reason as we induced the first approximate equation, that is, by reason that we expect to find $\phi_{1}$ such that it is possible under condition (A). We also neglect the derivatives of $p_{m-1}$. Then we get (1.2.3) and (1.2.4), where $H_{\phi}=\nabla^{2} \phi_{1}$ and $H_{p}=\nabla^{2} p_{m}$.

$$
\begin{aligned}
& (d / d t) \phi_{1}+p_{m}+p_{m-1}+2^{-1} i\left\langle\nabla p_{m}, J_{1} \nabla \phi_{1}\right\rangle+8^{-1} \operatorname{Tr}\left(J_{1} H_{p} J_{1} H_{\phi}\right) \\
& \quad+8^{-1}\left\langle\nabla \phi_{1}, J_{1} H_{p} J_{1} \nabla \phi_{1}\right\rangle=0 .
\end{aligned}
$$

$$
\begin{aligned}
& (d / d t)\left(J_{1} \nabla \phi_{1}\right)+J_{1} \nabla p_{m}+2^{-1} i J_{1} H_{p} J_{1} \nabla \phi_{1}-2^{-1} i J_{1} H_{\phi} J_{1} \nabla p_{m} \\
& \quad+4^{-1} J_{1} H_{\phi} J_{1} H_{p} J_{1} \nabla \phi_{1}=0 .
\end{aligned}
$$

$$
\begin{aligned}
& (d / d t)\left(J_{1} H_{\phi}\right)+J_{1} H_{p}+2^{-1} i\left(J_{1} H_{p}\right)\left(J_{1} H_{\phi}\right)-2^{-1} i\left(J_{1} H_{\phi}\right)\left(J_{1} H_{p}\right) \\
& \quad+4^{-1} J_{1} H_{\phi} J_{1} H_{p} J_{1} H_{\phi}=0 .
\end{aligned}
$$


We put $X=i J_{1} H_{\phi}$ and $A=i J_{1} H_{p}$. We assume that $X$ and $A$ are commutative. Then we get (1.2.5).

$$
(d / d t) X+A-4^{-1} A X^{2}=0 .
$$

Since we should get $\phi_{1}$ such that $\left.\phi_{1}\right|_{t=0}=0$, the initial condition of (1.2.5) must be (1.2.6).

$$
\left.X\right|_{t=0}=0 .
$$

We know that the solution of the initial value problem of the matrix valued ordinary differential equation (1.2.5) and (1.2.6) is given by (1.2.7) if it is well defined.

$$
X=-2 \tanh (A t / 2) .
$$

Using this solution we solve (1.2.2) and (1.2.3) as if $\phi_{1}, \nabla \phi_{1}$ and $\nabla^{2} \phi_{1}$ were independent of each other. We put $y=i J_{1} \nabla \phi_{1}$ and $b=i J_{1} \nabla p_{m}$. (1.2.3) implies (1.2.8).

$$
\begin{aligned}
& (d / d t) y+b+2^{-1} A y-2^{-1} X b-4^{-1} A X y=0 \\
& \left.y\right|_{t=0}=0 .
\end{aligned}
$$

(1.2.9) is the solution of (1.2.8).

$$
y=A^{-1} X b .
$$

We get (1.2.10) by (1.2.2).

(1.2.10) $(d / d t) \phi_{1}+p_{m}+p_{m-1}+2^{-1} i \sigma^{1}(b, y)-8^{-1} i \sigma^{1}(y, A y)-8^{-1} \operatorname{Tr}(X A)=0$.

Thus this implies (1.2.11) replacing $y$ by (1.2.9).

(1.2.11) $(d / d t) \phi_{1}+p_{m}+p_{m-1}+2^{-1} i \sigma^{1}\left(b, A^{-1} X b\right)-8^{-1} i \sigma^{1}\left(A^{-1} X b, X b\right)$

$$
-8^{-1} \operatorname{Tr}(X A)=0 \text {. }
$$

If we note (1.2.13) and (1.2.14), we get (1.2.12).

$$
\begin{gathered}
\text { (1.2.12) } \phi_{1}=-\left(p_{m}+p_{m-1}\right) t+2^{-1} i \sigma^{1}\left(b, A^{-1}\left(A^{-1} X+t\right) b\right)+8^{-1} \int_{0}^{t} \operatorname{Tr}(X A) d s . \\
\text { (1.2.13) } \sigma^{1}\left(b, A^{-1} X b\right)=0 . \quad\left(\sigma^{1}\left(u, A^{-1} X v\right)=\sigma^{1}\left(A^{-1} X u, v\right) .\right) \\
\text { (1.2.14) } 8^{-1} \int_{0}^{t} X^{2} d s=2^{-1} A^{-1} X+2^{-1} t I .
\end{gathered}
$$

By the way we know (1.2.15) and (1.2.16), where $F(\lambda)$ is defined by $(0.2 .4)$.

$$
2^{-1} i A^{-1}\left(A^{-1} X+t\right)=-F(A t / 2)(t / 2)^{2} \text {. }
$$




$$
\begin{gathered}
8^{-1} \int_{0}^{t} \operatorname{Tr}(A X) d s=-4^{-1} \operatorname{Tr} \int_{0}^{t} \tanh (A t / 2) A d s \\
=-2^{-1} \operatorname{Tr}(\log [\cosh (A t / 2)])
\end{gathered}
$$

Substituting them in (1.2.12) we get $\phi_{1}$ of (1.2.1).

Now we consider about $F(A t / 2)$ and $\log (\cosh A t / 2)$. They are defined in the form of Dunford integral (1.2.17) using the resolvent $(\lambda-A)^{-1}$ of $A$.

$$
f(A)=(2 \pi i)^{-1} \int_{\Gamma} f(\lambda)(\lambda-A)^{-1} d \lambda,
$$

where $f(\lambda)$ is a holomorphic function on a neighborhood $\Omega$ of the eigenvalues of $A$ and $\Gamma$ is a contour which is included in $\Omega$ and rounds the eigenvalues of $A$. It is necessary to point out the place, where the eigenvalues of $A$ exist, in order to use this definition. Since the Hesse matrix $H_{p}=\nabla^{2} p_{m}$ is non-negative on the characteristic set $\Sigma$ of $p_{m}$, all eigenvalues of $A$ lie on the real axis. However $H_{p}=\nabla^{2} p_{m}$ is not always non-negative out side of $\Sigma$. Therefore they swell out onto the complex plane. We estimate the width.

Proposition 1.2. Let $G$ be a real symmetric matrix on $\mathbb{C}^{2 n}$ and $J$ be a real unitary matrix such that $J^{2}=-I$. We assume that $G+\delta_{0} I \geqq 0$ for a real $\delta_{0}$. If $\delta_{0} \leqq|\operatorname{Im} \lambda|^{2}\left\{2 B+2\left(B^{2}+3|\operatorname{Im} \lambda|^{2}\right)^{1 / 2}\right\}^{-1}$ and $\operatorname{Im} \lambda \neq 0$, where $B$ $=\sup _{f \neq 0}\langle G f, f\rangle \mid\langle f, f\rangle$, then there exists the resolvent $(\lambda-i J G)^{-1}$ of $i J G$ such that (1.2.18) holds for $0<k<1$ if $\lambda$ satisfies (1.2.19) and $\operatorname{Im} \lambda \neq 0$.

$$
\begin{aligned}
& \left\|(\lambda-i J G)^{-1}\right\| \\
& \quad \leqq\left(4 B^{2}+5 k^{2}|\operatorname{Im} \lambda|^{2}\right)^{1 / 2}\left\{k(1-k)|\operatorname{Im} \lambda|^{2}\right\}^{-1}, \quad \text { when } B>0,
\end{aligned}
$$

or

$$
\begin{aligned}
& \leqq 2^{1 / 2}\{|\operatorname{Im} \lambda|(1-k)\}^{-1}, \quad \text { when } B \leqq 0 . \\
& k^{2}|\operatorname{Im} \lambda|^{2}\left\{2 B+2\left(B^{2}+3 k^{2}|\operatorname{Im} \lambda|^{2}\right)^{1 / 2}\right\}^{-1} \geqq \delta_{0} .
\end{aligned}
$$

Remark. We shall prove Proposition 1.2 at Appendix.

We may identify $T\left(T^{*} \mathbb{R}^{n}\right)$ and $\mathbb{R}^{2 n} \times \mathbb{R}^{2 n}$. We denote its element by $(X, Y)$, where $X=(x, \xi)$ belonging to $T^{*} \mathbb{R}^{n}$ and $Y=(y, \eta)$ belongieng to $T_{0}\left(T^{*} \mathbb{R}^{n}\right)$. We also use the notation that $x_{j}=X_{j}, \xi^{j}=X^{j}, y_{j}=Y_{j}$ and $\eta^{j}=Y^{j}$. Let $\chi$ and $c$ stand for a mapping on $T_{X}\left(T^{*} \mathbb{R}^{n}\right)$ and a mapping from $T_{X}\left(T^{*} \mathbb{R}^{n}\right)$ to $T^{*} \mathbb{R}^{n}$, which are defined by $(1.2 .20)$ and (1.2.21), respectively.

$$
\begin{gathered}
\chi(Y)=\chi_{X}(Y)=\left(\langle\xi\rangle^{-1 / 2} y,\langle\xi\rangle^{+1 / 2} \eta\right) . \\
\iota(Y)=\iota_{X}(Y)=Y .
\end{gathered}
$$


Let $p$ belong to $L_{1 / 2}^{m}$. We get (1.2.22) by the Tayler expansion, where $Y^{\prime}=\iota \chi(Y)$.

(1.2.22) $p\left(X+Y^{\prime}\right)=p(X)+\langle\nabla p(X), Y\rangle+2^{-1}\left\langle\nabla^{2} p(X) Y, Y\right\rangle+g(X, Y)$.

$$
g(X, Y)=2^{-1} \int_{0}^{1} \nabla^{3} p\left(X+\theta Y^{\prime}\right)(Y)(1-\theta)^{2} d \theta
$$

Proposition 1.3. If $p$ belongs to $L_{1 / 2}^{m}$ and $p \geqq 0$, then we get (1.2.24) for a constant $c_{0}$.

$$
\inf _{\|u\|=1}\left\langle\nabla^{2} p(X) u, u\right\rangle \geqq-c_{0} p(X)^{1 / 3}\langle\xi\rangle^{2 m / 3-1} .
$$

Proof. We assume that $\|u\|=1$ and $\left.\left\langle\nabla^{2} p u, u\right\rangle=-\delta_{0},\left(\delta_{0}\right\rangle 0\right)$. Substituting $Y=\mu u$ at (1.2.22) we get (1.2.25), where $\mu$ is a constant.

$$
\begin{aligned}
& 0 \leqq p+\mu\langle\nabla p, u\rangle+2^{-1} \mu^{2}\left\langle\nabla^{2} p u, u\right\rangle+g(\mu u) . \\
& g(\mu u)=2^{-1} \mu^{3} \int_{0}^{1} \nabla^{3} p(X+\theta \mu<\chi u)(u)(1-\theta)^{2} d \theta .
\end{aligned}
$$

If we assume (1.2.27), $g(\mu u)$ is estimated as (1.2.28) because $2\langle\xi\rangle \geqq \sum_{j=1}^{n} \mid(X$ $+\theta \mu \iota \chi u)^{j} \mid+1 \geqq\langle\xi\rangle / 2$.

$$
|\mu| \leqq 2^{-1}\langle\xi\rangle^{1 / 2} \text {. }
$$

$$
|g(\mu u)| \leqq c \mu^{3}\langle\xi\rangle^{m-3 / 2} \text {. }
$$

Particularly we put $\mu=\varepsilon_{0} p(X)^{1 / 3}\langle\xi\rangle^{-m / 3+1 / 2}$. If a positive number $\varepsilon_{0}$ is sufficiently small, (1.2.27) holds. So we get (1.2.29).

$$
2^{-1} \delta_{0} \mu^{2} \leqq p \pm \mu\langle\nabla p, u\rangle+c \varepsilon_{0}^{3} p .
$$

This implies (1.2.30) and so (1.2.31).

$$
\begin{aligned}
& 2^{-1} \delta_{0} \mu^{2} \leqq\left(1+c \varepsilon_{0}^{3}\right) p . \\
& \delta_{0} \leqq 2 \mu^{-2}\left(1+c \varepsilon_{0}^{3}\right) p \\
& \quad=2 \varepsilon_{0}^{-2} p^{-2 / 3}\langle\xi\rangle^{2 m / 3-1}\left(1+c \varepsilon_{0}^{3}\right) p \\
& \quad=c_{0} p^{1 / 3}\langle\xi\rangle^{2 m / 3-1}, c_{0}=2 \varepsilon_{0}^{-2}\left(1+c \varepsilon_{0}^{3}\right) .
\end{aligned}
$$

Combining Propositions 1.2 and 1.3 we get Proposition 1.4.

Proposition 1.4. Let $p$ belong to $L_{1 / 2}^{m}$ and $p \geqq 0$. There exists a constant $c_{1}$ such that (1.2.33) holds if (1.2.32) holds.

$$
c_{1} p^{1 / 6}\langle\xi\rangle^{5 m / 6-1} t \leqq|\operatorname{Im} \lambda|,(t>0) .
$$


$(1.2 .33) \quad\left\|(\lambda-S)^{-1}\right\| \leqq 8\left(\|S\|^{2}+|\operatorname{Im} \lambda|^{2}\right)^{1 / 2}|\operatorname{Im} \lambda|^{-2}$,

where $S=A t=i J_{1} \nabla^{2} p t$.

Proof. It is enough to prove it when $t=1$. We apply Proposition 1.2 to the case that $G=\nabla^{2} p(X), \delta_{0}=c_{0} p(X)^{1 / 3}\langle\xi\rangle^{2 m / 3-1}$ and $B=\sup _{f \neq 0}\langle G f, f\rangle \mid$ $\langle f, f\rangle$, where $c_{0}$ is the one in Proposition 1.3. We get immediately (1.2.35) under the condition (1.2.34).

(1.2.34) $k^{2}|\operatorname{Im} \lambda|^{2}\left\{2 B+2\left(B^{2}+3 k^{2}|\operatorname{Im} \lambda|^{2}\right)^{1 / 2}\right\}^{-1} \geqq c_{0} p(X)^{1 / 3}\langle\xi\rangle^{2 m / 3-1}$.

(1.2.35) $\left\|(\lambda-S)^{-1}\right\| \leqq\left(4 B^{2}+5 k^{2}|\operatorname{Im} \lambda|^{2}\right)^{1 / 2}\left\{k(1-k)|\operatorname{Im} \lambda|^{2}\right\}^{-1}, \quad(B>0) ，$

or

$$
\leqq 2^{1 / 2}\{|\operatorname{Im} \lambda|(1-k)\}^{-1}, \quad(B \leqq 0) .
$$

Lemma 1.5. Let $\alpha, \beta$ and $\mu$ be non-negative. If they satisfy (1.2.36), then the inequality (1.2.37) holds.

$$
\begin{gathered}
\alpha>\mu^{2}\left\{2 \beta+2\left(\beta^{2}+\mu^{2}\right)^{1 / 2}\right\}^{-1} . \\
\mu<2 \alpha+2(\alpha \beta)^{1 / 2} .
\end{gathered}
$$

In Lemma 1.5 we put $\alpha=3 c_{0} p(X)^{1 / 3}\langle\xi\rangle^{2 m / 3-1}, \beta=|B|, \mu=3^{1 / 2} k|\operatorname{Im} \lambda|$ and $k=1 / 2$. Then we get (1.2.38) for a constant $c$ since $\alpha+\beta \leqq c^{\prime}\langle\xi\rangle^{m-1}$.

$$
\mu<c \alpha^{1 / 2}\langle\xi\rangle^{m / 2-1 / 2}=(3 / 4)^{1 / 2} c_{1} p^{1 / 6}\langle\xi\rangle^{5 m / 6-1} .
$$

Thus we conclude that (1.2.34) holds if (1.2.39) holds.

$$
|\operatorname{Im} \lambda| \geqq c_{1} p^{1 / 6}\langle\xi\rangle^{5 m / 6-1} .
$$

Therefore (1.2.35) holds as $k=1 / 2$. For any $B(1.2 .35)$ implies (1.2.33) putting $k=1 / 2$ because $4\left(4 B^{2}+(5 / 4)|\operatorname{Im} \lambda|^{2}\right)^{1 / 2} \leqq 8\left(B^{2}+|\operatorname{Im} \lambda|^{2}\right)^{1 / 2}$ and $8^{1 / 2}<8\left(B^{2}\right.$ $\left.+|\operatorname{Im} \lambda|^{2}\right)^{1 / 2}|\operatorname{Im} \lambda|^{-1}$.

q.e.d.

Proof of Lemma 1.5. Since $\beta+\left(\beta^{2}+\mu^{2}\right)^{1 / 2} \leqq 2 \beta+\mu, \quad \alpha>\mu^{2}(4 \beta+2 \mu)^{-1}$. So we get that $\mu^{2}<2 \alpha \mu+4 \alpha \beta$. This implies that $\mu<\alpha+\left(\alpha^{2}+4 \alpha \beta\right)^{1 / 2} \leqq 2 \alpha$ $+2(\alpha \beta)^{1 / 2}$.

q.e.d.

Let us use the definition (1.2.17) for a holomorphic function $g$ on the closed domain $D=\{\lambda=a+i b ;|b|-|a| \leqq 1 / 2\}$ of $\mathbb{C}$. $g(S)$ is well defined if the eigenvalues of $S$ lie in the zonal domain $\{\lambda ;|\operatorname{Im} \lambda|<1 / 2\}$.

Proposition 1.6. Let $\Omega_{\mu}$ be a neighborhood of the characteristic set $\Sigma$ of $p_{m}$ such that 


$$
\Omega_{\mu}=\left\{X ; t p_{m}(X)^{1 / 6}\langle\xi\rangle^{5 m / 6-1} \leqq \mu\right\} .
$$

$g$ is a holomorphic function on D such that

(1.2.41) $|g(\lambda)| \leqq c(1+|\lambda|)^{k}$ on $D$ for some constants $c$ and $k \geqq 0$.

1) There exists $\mu>0$ such that $g($ At/2) is well defined if $X$ belongs to $\Omega_{\mu}$, where $A=i J_{1} \nabla^{2} p_{m}$ and $g(A t / 2)$ is given by (1.2.17).

2) On the domain $\Omega_{\mu}$ of 1$), g(A t / 2)$ has the estimate (1.2.42) for integers $l \geqq 0$.

$$
\left\|\nabla^{l} g(A t / 2)\right\| \leqq c_{l}\left(1+t\langle\xi\rangle^{m-1}\right)^{2 l+k+1}\langle\xi\rangle^{-l / 2} .
$$

Proof. 1) Let $c_{1}$ be the one in (1.2.32) of Proposition 1.4. We fix a parameter $\mu$ such that $0<c_{1} \mu<1$. Then the eigenvalues of $A t / 2$ lie on the zonal domain $\{\lambda ;|\operatorname{Im} \lambda|<1 / 2\}$ by Proposition 1.4. Thus 1$)$ is valid.

2) Let the another domain $D_{a}^{\prime}$ be defined such that $D_{a}^{\prime}=\{\lambda ;|\operatorname{Re} \lambda| \leqq 2 a+1\}$. $(\lambda-S)^{-1}$ is holomorphic in $\lambda$ out side $\left\{\lambda ;|\operatorname{Im} \lambda| \leqq c_{1} \mu / 2<1 / 2\right.$ and $\left.|\operatorname{Re} \lambda| \leqq\|S\|\right\}$, where $S=A t / 2$, and satisfies the estimate (1.2.33) and (1.2.43).

$$
\left\|(\lambda-S)^{-1}\right\| \leqq(|\lambda|-\|S\|)^{-1} \quad \text { if } \quad|\lambda|>\|S\| .
$$

We take a contour $\Gamma$ in (1.2.17) such that $\Gamma=\partial\left(D \wedge D^{\prime}{ }_{\|S\|}\right)$. We put $\Gamma_{1}=\Gamma \wedge \partial D$ and $\Gamma_{2}=\Gamma \wedge \partial D^{\prime}{ }_{\|S\|}$. Since $|\operatorname{Im} \lambda| \leqq 2\|S\|+3 / 2$ on $\gamma,(1.2 .33)$ and (1.2.43) imply (1.2.44) and (1.2.45).

$$
\begin{aligned}
& \left\|(\lambda-S)^{-1}\right\| \leqq c(1+\|S\|)|\operatorname{Im} \lambda|^{-2} \quad \text { on } \Gamma_{1} . \\
& \left\|(\lambda-S)^{-1}\right\| \leqq c(1+\|S\|)^{-1} \quad \text { on } \quad \Gamma_{2} .
\end{aligned}
$$

We get (1.2.46) and (1.2.47) for integers $l \geqq 0$ noting that $\left\|\nabla^{l} S\right\| \leqq c_{l} t\langle\xi\rangle^{m-1-l / 2}$ because $p_{m}$ belongs to $L_{1 / 2}^{m}$.

$$
\begin{aligned}
& \left\|\nabla^{l}(\lambda-S)^{-1}\right\| \\
& \quad \leqq c_{l}\left(1+t\langle\xi\rangle^{m-1}\right)^{l}(1+\|S\|)^{l+1}\langle\xi\rangle^{-l / 2}|\operatorname{Im} \lambda|^{-2(l+1)} \quad \text { on } \Gamma_{1} .
\end{aligned}
$$

(1.2.47) $\left\|\nabla^{l}(\lambda-S)^{-1}\right\|$

$$
\leqq c_{l}\left(1+t\langle\xi\rangle^{m-1}\right)^{l}(1+\|S\|)^{-l-1}\langle\xi\rangle^{-1 / 2} \quad \text { on } \quad \Gamma_{2} .
$$

Now we estimate $\nabla^{l} g(A t / 2)=(2 \pi i)^{-1} \int_{\Gamma} g(\lambda) \nabla^{l}(\lambda-S)^{-1} d \lambda$.

$$
\begin{aligned}
& \left\|\nabla^{l} g(A t / 2)\right\| \leqq(2 \pi)^{-1} \int_{\Gamma}|g(\lambda)|\left\|\nabla^{l}(\lambda-S)^{-1}\right\| d \lambda \\
& \quad \leqq c_{l} \int_{\Gamma_{1}}(1+\|S\|)^{l+k+1}\left(1+t\langle\xi\rangle^{m-1}\right)^{l}\langle\xi\rangle^{-l / 2}|\operatorname{Im} \lambda|^{-2(l+1)} d \lambda
\end{aligned}
$$




$$
\begin{aligned}
& +c_{l} \int_{\Gamma_{2}}(1+\|S\|)^{k-l-1}\left(1+t\langle\xi\rangle^{m-1}\right)^{l}\langle\xi\rangle^{-l / 2} d \lambda \\
\leqq & c_{l}\left\{(1+\|S\|)^{l+l+1}\left(1+t\langle\xi\rangle^{m-1}\right)^{l}\langle\xi\rangle^{-l / 2} \int_{1 / 2}^{2\|S\|+3 / 2} \sigma^{-2(l+1)} d \sigma\right. \\
& \left.+(1+\|S\|)^{k-l-1}\left(1+t\langle\xi\rangle^{m-1}\right)^{l}\langle\xi\rangle^{-l / 2} \int_{-2\|S\|-3 / 2}^{2\|S\|+3 / 2} d \sigma\right\} \\
\leqq & c_{l}(1+\|S\|)^{l+k+1}\left(1+t\langle\xi\rangle^{m-1}\right)^{l}\langle\xi\rangle^{-l / 2} \\
\leqq & c_{l}\left(1+t\langle\xi\rangle^{m-1}\right)^{2 l+k+1}\langle\zeta\rangle^{-l / 2}
\end{aligned}
$$

where the constants $c_{l}$ are changed suitably.

q.e.d.

Examples. $\tanh \lambda, \quad \lambda^{-1} \tanh \lambda, \quad i F(\lambda)=\lambda^{-1}\left(1-\lambda^{-1} \tanh \lambda\right), \quad \cosh \lambda, \quad \log$ $(\cosh \lambda),\left(\lambda^{-1} \tanh \lambda\right)^{1 / 2}, F(\lambda)\left(1+\left(\lambda^{-1} \tanh \lambda\right)^{1 / 2}\right)^{-1}, \lambda F(\lambda)\left(1+\left(\lambda^{-1} \tanh \lambda\right)^{1 / 2}\right)^{-1}$ and $(\exp (-2 \lambda \alpha)+1)(\exp (-2 \lambda)+1)^{-1}$, where $0 \leqq \alpha \leqq 1$.

We use the function $g(S)$ on $\operatorname{supp} \psi_{k}(k=1,2)$ which are defined by $(0.2 .7)$. Lemma 1.7 guarantees it.

Lemma 1.7. Let $\delta$ and $\varepsilon$ be those appearing in the definition of $\psi_{h}$. If $\delta<(1-2 \varepsilon) / 6$, there exists $T>0$ such that $\operatorname{supp} \psi_{k}(k=1,2)$ is included in $\Omega_{\mu}$ for $t$ of $[0, T]$. Therefore the eigenvalues of $S=A t / 2$ lie on the zonal domain $\{\lambda ;|\operatorname{Im} \lambda|<1 / 2\}$ and also $g(S)$ of Proposition 1.6 is well defined on supp $\psi_{k}$ and has the estimate (1.2.42).

Proof. $\operatorname{supp} \psi_{1}$ is included in $\operatorname{supp} \psi_{2}=\left\{p_{m}\langle\xi\rangle^{1-m-2 \varepsilon} \leqq 4\right\} \wedge\left\{t\langle\xi\rangle^{m-1-\delta}\right.$ $\leqq 4\} . \quad Z=t p_{m}^{1 / 6}\langle\xi\rangle^{5 m / 6-1} \leqq 2^{1 / 3} t\langle\xi\rangle^{m-1+(2 \varepsilon-1) / 6}$. If $m-1+(2 \varepsilon-1) / 6<0$, it is sufficient to take $T=\mu 2^{-1 / 3}$. If $m-1+(2 \varepsilon-1) / 6 \geqq 0$, then $m-1-\delta>0$.

$$
\begin{gathered}
Z \leqq 2^{1 / 3} t^{((1-2 \varepsilon) / 6-\delta) /(m-1-\delta)}\left(t\langle\xi\rangle^{m-1-\delta}\right)^{(m-1+(2 \varepsilon-1) / 6) /(m-1-\delta)} \leqq 2^{1 / 3+2 \alpha} t^{\beta} \\
\alpha=(m-1+(2 \varepsilon-1) / 6) /(m-1-\delta) \geqq 0 \quad \text { and } \quad \beta=((1-2 \varepsilon) / 6-\delta) /(m-1-\delta)>0 .
\end{gathered}
$$
Thus we also get $T>0$ which satisfies that $2^{1 / 3+2 \alpha} T^{\beta} \leqq \mu$. q.e.d.

In conclusion we state the following proposition to finish this section.

Proposition 1.8. $\phi_{1}$ of (1.2.1) is well defined on supp $\psi_{k}, k=1,2$, if $\delta<(1-2 \varepsilon) / 6$ and if $t$ is sufficiently small.

\section{§1.3. A Class of Pseudodifferential Operators}

We introduce a kind of classes for symbols which is convinient to construct the parametrix at a neighborhood of the characteristic set $\Sigma$ of $p_{m}$. 
Let $\Omega_{0}$ be a domain such that

$$
\Omega_{0}=\left\{(t, x, \xi) ; p_{m} \leqq 8\langle\xi\rangle^{m-1+2 \varepsilon} \text { and } t\langle\xi\rangle^{m-1-\delta} \leqq 8\right\} \text {. }
$$

We define $N(j, k, l)$ a subspace of $C^{+\infty}\left(\Omega_{0}\right)$ through the four steps (1.3.2-5), where $j$ and $k$ are integers, and $l$ is a real number.

(1.3.2) $f$ belongs to $N(0,0, l)$ if and only if for any integers $\alpha$ and $\beta \geqq 0$ there exist constants $c(\alpha, \beta)$ and $d(\alpha, \beta)$ such that

$$
\left|\partial_{t}^{\alpha} \nabla^{\beta} f\right| \leqq c(\alpha, \beta)\left(1+t\langle\xi\rangle^{m-1}\right)^{d(\alpha, \beta)}\langle\xi\rangle^{l-\beta \varepsilon+\alpha(m-1)} \quad \text { on } \Omega_{0} .
$$

(1.3.3) When $j \geqq 0$ and $k \geqq 0, f$ belongs to $N(j, k, l)$ if and only if

$$
f=\left(t\langle\xi\rangle^{m-1}\right)^{j-k} g\left(t J_{1} \nabla p_{m}\right),
$$

where $g(\zeta)$ is a polynomial of homogeneous order $k$ in $\zeta$ with coefficients in $N(0,0, l)$.

$$
\begin{gathered}
N(j, k, l)=N(j, 0, l+k \varepsilon) \text { when } j \geqq 0 \text { and } k<0 . \\
N(j, k, l)=N(0, k, l) \text { when } j<0 .
\end{gathered}
$$

We get immediately Proposition 1.9 .

\section{Proposition 1.9.}

$$
N(j, k, l) \text { are } N(0,0,0) \text {-modules. }
$$

$$
N(j, k, l) \text { is included in } N(j, k-1, l+\varepsilon) \text {. }
$$

$N(j, k, l)$ is included in $N(j-1, k, l)$.

If $f$ belongs to $N(j, k, l)$, then we get (1.3.9-11).

(1.3.9) $\nabla f$ belongs to $N(j, k-1, l)+N(j, k, l-\varepsilon)$ which is included in $N(j, k-1, l)$.

$$
\partial_{t} f \text { belongs to } N(j-1, k, m-1+l) \text {. }
$$

$$
\int_{0}^{t} f d t \text { belongs to } N(j+1, k, l-m+1) \text {. }
$$

Remark. It is not necessary for the domain of functions in the definition of $N(j, k, l)$ to be restricted to $\Omega_{0}$. We may define all relation on $\mathbf{R}^{n}$ uniformly, though we can not expect that (1.3.7) and the inclusion at the last part of (1.3.9) hold. We denote it by $N_{g}(j, k, l)$. When we construct the parametrix out side of the characteristic set $\Sigma$ or in the whole space, we will use this notation. 
Let $H_{i}(i=1,2,3)$ stand for subspaces of $C^{-\infty}[0,+\infty)$ which are defined by $(1.3 .13)$.

(1.3.13) $H_{1}=\{h ; \operatorname{supp}(d / d t) h$ is included in $[1,2]\}$, $H_{2}=\left\{h ; \operatorname{supp} h\right.$ is included in $[1,+\infty)$ and $h$ belongs to $\left.H_{1}\right\}$

and

$$
H_{3}=\{h ; \operatorname{supp} h \text { is included in }[1,2]\} .
$$

We put $h_{1}=\psi$, where $\psi$ is the onc in (0.2.7). Then $h_{1}$ belongs to $H_{1}$ and $\left(1-h_{1}\right)^{\alpha}$ belongs to $H_{2}$ for $\alpha>0$. $\quad h_{1}$ satisfies (1.3.14) for all $n \geqq 1$, for all $\alpha$ such that $1>\alpha \geqq 0$ and for some $h_{(n, \alpha)}$ belonging to $H_{3}$.

$$
(d / d t)^{n} h_{1}=h_{(n, \alpha)}\left(1-h_{1}\right)^{\alpha} .
$$

And also $\left(1-h_{1}\right)^{\alpha}$ satisfies (1.3.15) for all $\beta$ such that $\alpha>\beta \geqq 0$ and for some $h_{(\alpha, \beta)}$ belonging to $H_{3}$.

$$
(d / d t)\left(1-h_{1}\right)^{\alpha}=h_{(\alpha, \beta)}\left(1-h_{1}\right)^{\beta} .
$$

Next we define subspaces $H_{i}(\mu)$ of $H_{i}(i=1,2,3)$ for $+\infty \geqq \mu \geqq 0$.

$$
H_{i}(0)=H_{i} .
$$

(1.3.17) $H_{i}(\mu)$ consists of elements $h$ of $H_{i}$ such that $h=h_{(\alpha)}\left(1-h_{1}\right)^{\alpha}$ for any $\alpha$ such that $\mu>\alpha \geqq 0$ and for some $h_{(\alpha)}$ belonging to $H_{i}$, if $\mu>0$.

They have the following properties.

$H_{i}(\mu)$ is included in $H_{i}\left(\mu^{\prime}\right)$ if $\mu>\mu^{\prime}$.

(1.3.19) $(d / d t)^{n} h$ belongs to $H_{3}(\mu)$ for $n \geqq 1$ if $f$ belongs to $H_{i}(\mu)(i=1,2,3)$.

$$
\begin{aligned}
& H_{1}(\mu)=H_{2}(\mu) \text { includes } H_{3}(\mu) \text { if } \mu>0 . \\
& H_{1}(\mu) H_{i}\left(\mu^{\prime}\right) \text { is included in } H_{i}\left(\mu+\mu^{\prime}\right) .
\end{aligned}
$$

Moreover we consider $\Psi_{1}(\mu)$ and $\Psi_{2}$ function spaces on $\Omega_{0}$ which are defined by (1.3.22) and (1.3.23), respectively.

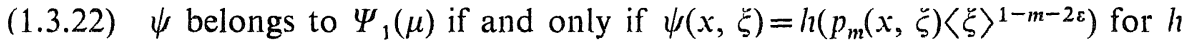
of $H_{1}(\mu)$.

(1.3.23) $\psi$ belongs to $\Psi_{2}$ if and only if $\psi$ vanishes on $\left\{t\langle\xi\rangle^{m-1-\delta} \leqq 1\right\}$.

We define $N(j, k, l, \mu)$, which are $N(0,0,0)$-modules generated by $\Psi_{1}(\mu) N(j, k, l)$, by $(1.3 .24)$ and $N^{-\infty}$ by (1.3.25), respectively. 


$$
\begin{aligned}
& N(j, k, l, \mu)=\Psi_{1}(\mu) N(j, k, l) . \\
& N^{-\infty}=\Psi_{2} \wedge \sum_{(j, k, l)} N(j, k, l) .
\end{aligned}
$$

We arrange their properties in Proposition 1.10 and 1.11 .

\section{Proposition 1.10.}

(1.3.26) $\Psi_{1}(\mu) \Psi_{1}\left(\mu^{\prime}\right)$ is included in $\Psi_{1}\left(\mu+\mu^{\prime}\right)$.

(1.3.27) $N(j, k, l, \mu) N\left(j^{\prime}, k^{\prime}, l^{\prime}, \mu^{\prime}\right)$ is included in $N\left(j+j^{\prime}, k+k^{\prime}, l+l^{\prime}, \mu+\mu^{\prime}\right)$.

(1.3.28) $N(j, k, l, \mu)$ is included in $N\left(j^{\prime}, k, l^{\prime}, \mu^{\prime}\right)$ if $j \geqq j^{\prime}, l \leqq l^{\prime}$ and $\mu \geqq \mu^{\prime}$.

(1.3.29) $N(j, k, l, \mu)$ is included in $N(j, k-1, l+\varepsilon, \mu)$. (Refer to Proposition 1.19.)

(1.3.30) $\Psi_{1}(\mu)$ is included in $N(0,0,0, \mu)$.

(1.3.31) If $\psi$ belongs to $\Psi_{1}(\mu)$, then $\nabla \psi$ belongs to $N(0,0,-1 / 2, \mu)$ $+N(0,1,-2 \varepsilon, \mu)$.

If $f$ belongs to $N(j, k, l, \mu)$, then we get (1.3.32-35).

(1.3.32) $f \nabla p_{m}$ belongs to $N(j, k+1, l+m-1, \mu)$.

(1.3.33) $\nabla$ f belongs to $N(j, k-1, l, \mu)$. ( $\nabla^{v} f$ belongs to $N(j, k-v, l, \mu)$.)

(1.3.34) $\partial_{t} f$ belongs to $N(j-1, k, l+m-1, \mu)$.

(1.3.35) $\int_{0}^{t} f d t$ belongs to $N(j+1, k, l-m+1, \mu)$.

(1.3.36) If $f$ belongs to $N^{-\infty}$, then $\nabla f, \partial_{t} f$ and $\int_{0}^{t} f d t$ belong to $N^{-\infty}$.

(1.3.37) $N^{-\infty} N(j, k, l, \mu)$ is included in $N^{-\infty}$.

Proposition 1.11. We get the relation $(1.3 .38-46)$ for $\psi_{1}^{1}, \psi_{1}^{2}$ and $\psi_{1}$ of (0.2.7).

(1.3.38) $\psi_{1}^{1}$ belongs to $\Psi_{1}(0)$ which is included in $N(0,0,0,0)$.

(1.3.39) $1-\psi_{1}^{2}$ belongs to $\Psi_{2}$ which is included in $N^{-\infty}$.

(1.3.40) $\left(1-\psi_{1}^{2}\right) N(j, k, l, \mu)$ is included in $N^{-\infty}$.

(1.3.41) $\left(1-\psi_{1}^{1}\right)$ belongs to $N(0,0,0,1)$.

(1.3.42) $\left(1-\psi_{1}^{1}\right) N(j, k, l, \mu)$ is included in $N(j, k, l, \mu+1)$. 
(1.3.43) $\psi_{1}=\psi_{1}^{1} \psi_{1}^{2}=\psi_{1}^{1}-\psi_{1}^{1}\left(1-\psi_{1}^{2}\right)$ belongs to $\psi_{1}^{1}+N^{-\infty}$ which is included in $N(0,0,0,0)+N^{-\infty}$.

(1.3.44) $1-\psi_{1}=1-\psi_{1}^{1}+\psi_{1}^{1}\left(1-\psi_{1}^{2}\right)$ belongs to $1-\psi_{1}^{1}+N^{-\infty}$ which is included in $N(0,0,0,1)+N^{-\infty}$.

(1.3.45) $\left(1-\psi_{1}\right) N(j, k, l, \mu)$ is included in $N(j, k, l, \mu+1)+N^{-\infty}$.

(1.3.46) $\nabla \psi_{1}$ belongs to $-\nabla\left(1-\psi_{1}^{1}\right)+\nabla N^{-\infty}$ which is included in $N(0,-1,0,1)+N^{-\infty}$.

Remark. $\quad N_{g}(j, k, l, \mu)$ and $N_{g}^{-\infty}$ are also well defined as $\Psi_{1}(\mu) N_{g}(j, k, l)$ and $\Psi_{2} \wedge \sum_{(j, k, l)} N_{g}(j, k, l)$, respectively. They satisfy Proposition 1.10-11 without (1.3.29) and (1.3.33). (1.3.33) should be left as (1.3.47).

(1.3.47) $\nabla f$ belongs to $N_{g}(j, k, l-\varepsilon, \mu)+N_{g}(j, k-1, l, \mu)$.

The relation between $N$ and $N_{g}$ is given by (1.3.49-50).

(1.3.48) $\quad \psi_{2} N(j, k, l, \mu)$ is included in $N_{g}(j, k, l, \mu)+N_{g}^{-\infty}$ and $N_{g}(j, k, l, \mu)$ is included in $N(j, k, l, \mu)$.

(1.3.49) $\psi_{2} N^{-\infty}$ is included in $N_{g}^{-\infty}$, which is included in $N^{-\infty}$.

We introduce some class by gathering $N$ or $N_{g}$ as (1.3.50-53).

$$
N^{*}(j, k, l, \mu)=\sum_{v \leq k} N(j, v, l, \mu) .
$$

It is clear that Proposition 1.10 and 1.11 are valid for $N^{*}(j, k, l, \mu)$ substituted in the place of $N(j, k, l, \mu)$.

$$
\begin{aligned}
& N^{* *}(j, k, l, \mu)=\sum_{v>0} N^{*}(j+v, k+v, l, \mu) . \\
& N_{g}^{*}(j, k, l, \mu)=\sum_{v \leqq k} N_{g}(j, v, l, \mu) . \\
& N_{g}^{* *}(j, k, l, \mu)=\sum_{v \geqq 0} N_{g}^{*}(j+v, k+v, l, \mu) .
\end{aligned}
$$

Finally we define $N_{g}(j, k, l$, out $)$, which consists of functions belonging to $N_{g}(j, k, l,+\infty)$ and supported on $\operatorname{supp}\left(1-\psi_{2}^{1}\right)$. We also define $N_{g}^{*}(j, k, l$, out $)$ and $N_{g}^{* * *}(j, k, l$, out $)$ in the same way as $(1.3 .52-53)$.

\section{§1.4. First Approximate Equation}

In this section we show that the complex phase function $\phi$ given in $(0.2 .6)$ satisfies the first approximate equation (1.1.4) in the sense of Proposition 1.12. We consider it only on supp $\psi_{2}$. 
$\phi=\psi_{1} \phi_{1}+\left(1-\psi_{1}\right) \phi_{2}=\phi_{1}+\left(1-\psi_{1}\right)\left(\phi_{2}-\phi_{1}\right)$

\section{Proposition 1.12.}

(1.4.2) $(d / d t) \phi+p_{m}+p_{m-1}+(2 i)^{-1} \sigma_{1}\left(\nabla p_{m}, \nabla \phi\right)$

$$
+(2 i)^{-2} 2^{-1} \sigma_{2}\left(\nabla^{2} p_{m}, \nabla \phi \nabla \phi+\nabla^{2} \phi\right)
$$

$\equiv 0, \quad \bmod N(3,3, m-1-\varepsilon, 0)+N(1,1, m-1-\varepsilon, 0)$

$$
+N(1,0, m-1-2 \varepsilon, 0)+N(2,2, m-1,1)+N(0,0, m-1,1)+N^{-\infty} \text {. }
$$

Proof. We reduce $\nabla \phi, \nabla^{2} \phi$ and $(d / d t) \phi$ to prove Proposition 1.12.

$$
\begin{aligned}
& \phi_{2}-\phi_{1} \\
= & t p_{m-1}+\sigma^{1}(b t / 2, F(\mathrm{~A} t / 2) b t / 2)+2^{-1} \operatorname{Tr}(\log [\cosh (A t / 2)])-t\langle\xi\rangle^{m-1},
\end{aligned}
$$

which belongs to $N(1,0,0,0)+N(2,2,0,0)$, because $\log [\cosh (\hat{\lambda})]=\lambda f(\lambda)$, where $f(\lambda)$ is bounded on the domain $D$ in Proposition 1.6. This implies (1.4.4-5) by (1.3.45) and (1.3.33).

(1.4.4) $\left(1-\psi_{1}\right)\left(\phi_{2}-\phi_{1}\right)$ belongs to $N(1,0,0,1)+N(2,2,0,1)+N^{-\infty}$.

(1.4.5) $\quad \nabla\left\{\left(1-\psi_{1}\right)\left(\phi_{2}-\phi_{1}\right)\right\}$ belongs to $N(1,-1,0,1)+N(2,1,0,1)+N^{-\infty}$.

Therefore we get (1.4.6-7) also using (1.3.33).

$$
\begin{gathered}
\nabla \phi \equiv \nabla \phi_{1}, \quad \bmod N(1,-1,0,1)+N(2,1,0,1)+N^{-\infty} . \\
\nabla^{2} \phi \equiv \nabla^{2} \phi_{1}, \quad \bmod N(1,-2,0,1)+N(2,0,0,1)+N^{-\infty} .
\end{gathered}
$$

We further reduce $\nabla \phi_{1}$ and $\nabla^{2} \phi_{1}$ noting the form of $\phi_{1}$.

(1.4.8) $\phi_{1}=-t p_{m}-t p_{m-1}-\sigma^{1}(b t / 2, F(A t / 2) b t / 2)-2^{-1} \operatorname{Tr}(\log [\cosh (A t / 2)])$.

Since $t p_{m-1}, F(A t / 2)$ and $\operatorname{Tr}(\log [\cosh (A t / 2)])$ belong to $N(1,0,0,0)$, we get (1.4.9-12), where $f_{0}(\lambda)=\lambda^{-1} \tanh \lambda\left(=1+\lambda^{2} f^{\sim}(\lambda)\right)$ and $f_{1}(\lambda)=\tanh \lambda$.

(1.4.9) $i J_{1} \nabla \phi_{1} \equiv-f_{0}(A t / 2) b t, \quad \bmod \quad N(3,2,-1 / 2,0)+N(1,0,-1 / 2,0)$.

(1.4.10) $i J_{1} \nabla \phi_{1}$ belongs to $N(1,1,0,0)+N(1,0,-1 / 2,0)$,

because $N(3,2,-1 / 2,0)$ is included in $N(1,1,0,0)$.

(1.4.11) $i J_{1} \nabla^{2} \phi_{1} \equiv-2 f_{1}(A t / 2), \quad \bmod N(2,1,-1 / 2,0)+N(1,-1,-1 / 2,0)$ ， because $N(3,1,-1 / 2,0)$ is included in $N(2,1,-1 / 2,0)$.

(1.4.12) $\quad \nabla^{2} \phi_{1}$ belongs to $N(1,0,0,0)+N(1,-1,-1 / 2,0)$.

In conclusion we get (1.4.13-17). 


$$
\begin{aligned}
& i J_{1} \nabla \phi \equiv-f_{0}(A t / 2) b t \\
& \quad \bmod N(3,2,-\varepsilon, 0)+N(1,0,-\varepsilon, 0)+N(2,1,0,1)+N^{-\infty}
\end{aligned}
$$

because $N(1,0,-1 / 2,0)$ and $N(1,-1,0,1)$ are included in $N(1,0,-\varepsilon, 0)$, where $\varepsilon \leqq 1 / 2$.

$$
\begin{aligned}
& i J_{1} \nabla^{2} \phi \equiv-2 f_{1}(A t / 2), \\
& \quad \bmod N(2,1,-\varepsilon, 0)+N(1,0,-2 \varepsilon, 0)+N(2,0,0,1)+N^{-\infty}
\end{aligned}
$$

because $N(1,-1,-1 / 2,0)+N(1,-2,0,1)$ are included in $N(1,0,-2 \varepsilon, 0)$.

$$
\begin{aligned}
& \sigma_{2}\left(\nabla^{2} p_{m}, \nabla^{2} \phi\right)=\operatorname{Tr}\left(i J_{1} \nabla^{2} p_{m} \cdot i J_{1} \nabla^{2} \phi\right) \equiv-2 \operatorname{Tr}\left[A f_{1}(A t / 2)\right] \\
& \quad \bmod N(2,1, m-1-\varepsilon, 1)+N(1,0, m-1-\varepsilon, 0) \\
& \quad+N(2,0, m-1,1)+N^{-\infty} . \\
& \sigma_{2}\left(\nabla^{2} p_{m}, \nabla \phi \cdot \nabla \phi\right)=-\left\langle\nabla \phi, J_{1} \nabla^{2} p_{m} J_{1} \nabla \phi\right\rangle \\
& \quad=i \sigma^{1}\left(i J_{1} \nabla \phi, i J_{1} \nabla^{2} p_{m} \cdot i J_{1} \nabla \phi\right) \\
& \quad \equiv i \sigma^{1}\left(f_{0}(A t / 2) b t, A f_{0}(A t / 2) b t\right) \\
& \quad \bmod N(4,3, m-1-\varepsilon, 0)+N(2,1, m-1-\varepsilon, 0) \\
& \quad+N(2,0, m-1-2 \varepsilon, 0)+N(3,2, m-1,1)+N^{-\infty}
\end{aligned}
$$

because $A i J_{1} \nabla \phi$ belongs to $N(1,1, m-1,0)+N(1,0, m-1-\varepsilon, 0)+N^{-\infty}$ and $N(4,2, m-1-2 \varepsilon, 0)+N(3,1, m-1-\varepsilon, 1)$ is included in $N(2,1, m-1-\varepsilon, 0)$.

$$
\begin{aligned}
\sigma_{1}\left(\nabla p_{m}, \nabla \phi\right) & =-\sigma^{1}\left(i J_{1} \nabla p_{m}, i J_{1} \nabla \phi\right) \\
& \equiv \sigma^{1}\left(b, f_{0}(A t / 2) b\right)=0,
\end{aligned}
$$

$\bmod N(3,3, m-1-\varepsilon, 0)+N(1,1, m-1-\varepsilon, 0)+N(2,2, m-1,1)+N^{-\infty}$, because $f_{0}(\lambda)$ is an even function. (Refer to Appendix.) Thus we get (1.4.18) from the above properties, where $f_{2}(\lambda)=\lambda^{-1}(\tanh \lambda)^{2}$.

$$
\begin{gathered}
(2 i)^{-1} \sigma_{1}\left(\nabla p_{m}, \nabla \phi\right)+(2 i)^{-2} 2^{-1} \sigma_{2}\left(\nabla^{2} p_{m}, \nabla \phi \nabla \phi+\nabla^{2} \phi\right) \\
\quad \equiv(2 i)^{-2} i \sigma^{1}\left(b, f_{2}(A t / 2) b\right) t-(2 i)^{-2} \operatorname{Tr}\left[A \cdot f_{1}(A t / 2)\right],
\end{gathered}
$$

$\bmod N(3,3, m-1-\varepsilon, 0)+N(1,1, m-1-\varepsilon, 0)+N(1,0, m-1-2 \varepsilon, 0)$ $+N(2,2, m-1,1)+N(2,0, m-1,1)+N^{-\infty}$,

because $\lambda f_{0}(\lambda)^{2}=f_{2}(\lambda), N(4,3, m-1-\varepsilon, 0)$ is included in $N(3,3, m-1-\varepsilon, 0)$, $N(2,1, m-1-\varepsilon, 0)$ is included in $N(1,1, m-1-\varepsilon, 0), N(2,0, m-1-2 \varepsilon, 0)$ is included in $N(1,0, m-1-2 \varepsilon, 0)$ and $N(3,2, m-1,1)$ is included in $N(2,2, m-1,1)$.

On the other hand we get (1.4.19-21) for $\partial_{t} \phi$.

$$
\partial_{t} \phi \equiv \partial_{t} \phi_{1}+\left(1-\psi_{1}\right)\left(\partial_{t} \phi_{2}-\partial_{t} \phi_{1}\right) \quad \bmod N^{-\infty}
$$


(1.4.20) $\partial_{t} \phi_{1}$

$$
=-p_{m}-p_{m-1}-(2 i)^{-2} i \sigma^{1}\left(b, f_{2}(A t / 2) b\right) t+(2 i)^{-2} \operatorname{Tr}\left[A \cdot f_{1}(A t / 2)\right],
$$

because $(d / d t)\left(t^{2} F(\lambda t)\right)=i^{-1} f_{2}(\lambda t) t$ and $(d / d t)(\log (\cosh \lambda t))=\lambda f_{1}(\lambda t)$.

$$
\begin{aligned}
& \partial_{t} \phi_{2}-\partial_{t} \phi_{1} \\
& \quad=p_{m-1}+(2 i)^{-2} i \sigma^{1}\left(b, f_{2}(A t / 2) b\right) t-(2 i)^{-2} \operatorname{Tr}\left[A \cdot f_{1}(A t / 2)\right]-\langle\xi\rangle^{m-1}
\end{aligned}
$$

which belongs to $N(2,2, m-1,0)+N(0,0, m-1,0)$. Therefore we get (1.4.22).

$$
\begin{aligned}
\partial_{t} \phi & \equiv-p_{m}-p_{m-1}-(2 i)^{-2} i \sigma^{1}\left(b, f_{2}(A t / 2) b\right) t+(2 i)^{-2} \operatorname{Tr}\left[A \cdot f_{1}(A t / 2)\right] \\
& \bmod N(2,2, m-1,1)+N(0,0, m-1,1)+N^{-\infty}
\end{aligned}
$$

Combining (1.4.18) and (1.4.22) we complete the proof of Proposition 1.12.

q.e.d.

\section{§1.5. Solution of Second Approximate Equation}

The coefficients of the second approximate equation (1.1.12) are not real in general though it is a linear and first order partial differential equation. We can not expect to find exact solutions. But it is interested and sufficiently effective to find approximate ones for defining the type of the parametrix.

Proposition 1.13. Let $g(\zeta)$ be an homogeneous polynormial of order $k$ in $\zeta$ with coefficients in $N(0,0,0,0)$ such that $g(\zeta)$ belongs to $N(j, k, l+m-1, \mu)$ if $\zeta$ is replaced by $b=i J_{1} \nabla p_{m}$. We define another polynomial $h(\zeta)$ by (1.5.1).

$$
\begin{aligned}
& h(\zeta)=\int_{0}^{t} g(s, x, \xi, \theta(t, s) \zeta) d s, \text { and } \\
& \quad \theta(t, s)=\{1+\exp (-A s)\}\{1+\exp (-A t)\}^{-1} .
\end{aligned}
$$

Then, $h=h\left(i J_{1} \nabla p_{m}\right)$ belongs to $N(j+1, k, l, \mu)$ and satisfies (1.5.2).

$$
\begin{aligned}
& (d / d t) h-2^{-1}\left\langle\nabla h,\left\{1-f_{1}(A t / 2)\right\} b\right\rangle-g \equiv 0, \\
& \quad \bmod N(j+1, k+1, l+m-1-\varepsilon, \mu), \text { where } f_{1}(\lambda)=\tanh \lambda
\end{aligned}
$$

We can take out a leading part of the proof as Lemma 1.14. Let $K(t)$ be a continuous function valued in $L\left(\mathbf{C}^{n}\right)$, linear mapping on $\mathbf{C}^{n}$, and $\theta(t, s)$ be the solution of the equation (1.5.3). 


$$
\begin{aligned}
& \left\{(d / d t)+{ }^{t} K(t)\right\}^{t} \theta(t, s)=0 . \\
& { }^{t} \theta(s, s)=I .
\end{aligned}
$$

We consider homogeneous polynomial $g(\lambda)$ and $h(\lambda)$ of order $k$ in $\zeta$. They satisfy (1.5.4).

$$
\begin{aligned}
& k g(\zeta)=\langle\zeta, G(\zeta)\rangle \text { and } k h(\zeta)=\langle\zeta, H(\zeta)\rangle \text {, where } G(\zeta)=\partial_{\zeta} g(\zeta) \\
& \text { and }(H \zeta)=\partial_{\zeta} g(\zeta) \text {. }
\end{aligned}
$$

Lemma 1.14. Let $h(\zeta)$ be defined by (1.5.5).

$$
h(\zeta)=\int_{0}^{t} g(s, \theta(t, s) \zeta) d s .
$$

$h(\zeta)$ is a solution of (1.5.6).

$$
\begin{aligned}
& (d / d t) h+\langle K(t) \zeta, H(\zeta)\rangle=g(\zeta) \\
& \left.h\right|_{t=0}=0 .
\end{aligned}
$$

$$
\begin{aligned}
\text { Proof. } \quad(d / d t) h \quad & =g(\zeta)+\int_{0}^{t}\langle(d / d t) \theta(t, s) \zeta, G(\theta(t, s) \zeta)\rangle d s \\
& =g(\zeta)-\int_{0}^{t}\langle\theta(t, s) K(t) \zeta, G(\theta(t, s) \zeta)\rangle d s \\
& =g(\zeta)-\left\langle K(t) \zeta, \int_{0}^{t} \theta(t, s) G(\theta(t, s) \zeta) d s\right\rangle \\
& =g(\zeta)-\langle K(t) \zeta, H(\zeta)\rangle,
\end{aligned}
$$

because $H(\zeta)=\int_{0}^{t} t \theta(t, s) G(\theta(t, s) \zeta) d s$.

q.e.d.

Proof of Proposition 1.13. Let us put $K(t)$ as (1.5.7) in Lemma 1.14.

$$
K(t)=2^{-1} A f_{1}(A t / 2)-2^{-1} A .
$$

Then we get $\theta(t, s)$ in (1.5.1) for the solution of (1.5.3). In fact the solution of (1.5.3) is given by $\exp \left(-\int_{s}^{t} K(r) d r\right)$ since $K(t)$ and $K(s)$ are commutative.

$$
-\int_{s}^{t} K(r) d r=A(t-s) / 2-\log (\cosh (A t / 2) / \cosh (A s / 2)) .
$$

So we get (1.5.9).

$$
\begin{aligned}
& \exp \left(-\int_{s}^{t} K(r) d r\right) \\
& =\exp (A(t-s) / 2)\{\exp (A s / 2)+\exp (-A s / 2)\}\{\exp (A t / 2) \\
& =\{1+\exp (-A s)\}\{1+\exp (-A t)\}^{-1}
\end{aligned}
$$


It is clear by definition that $h(b)$ belongs to $N(j+1, k, l, \mu)$ if $g(b)$ belongs to $N(j, k, l+m-1, \mu)$. For $\nabla h(b)$ we get (1.5.10).

$$
\nabla\{h(b)\}=h^{\sim}(b)+A^{t} H(b),
$$

where $h \sim(\zeta)=\nabla h(\zeta), H(\zeta)=\partial_{\zeta} h(\zeta)$ and $h \sim(b)$ belongs to $N(j+1, k, l-\varepsilon, \mu)$. This means (1.5.11) because $\left\{1-f_{1}(A t / 2)\right\} b$ belongs to $N(0,1, m-1,0)$.

$$
\begin{aligned}
& \left\langle\nabla h(b), 2^{-1}\left\{1-f_{1}(A t / 2)\right\} b\right\rangle \\
& \quad \equiv\left\langle H(b), 2^{-1} A\left\{1-f_{1}(A t / 2)\right\} b\right\rangle, \quad \bmod N(j+1, k+1, l+m-1-\varepsilon, \mu) \\
& =-\langle H(b), K(t) b\rangle=(d / d t) h(b)-g(b)
\end{aligned}
$$

Lemma 1.15. $h$ of $N(j+1, k, l, \mu)$ gotten in Proposition 1.13 satisfies (1.5.12) for a given $g$ of $N(j, k, l+m-1, \mu)$.

(1.5.12) $(d / d t) h+\sum_{v=1}^{2}(2 i)^{-v}(v !)^{-1}\left\{\sigma_{v}\left(p_{m}, h \exp \phi\right)\right.$

$$
\left.-h \sigma_{v}\left(p_{m}, \exp \phi\right)\right\} \exp (-\phi) \equiv g,
$$

$\bmod N(j+1, k-2, l+m-1, \mu)+N(j+1, k+1, l+m-1-\varepsilon, \mu)$

$$
+N(j+3, k, l+m-1, \mu+1)+N^{-\infty} \text {. }
$$

Proof.

$$
\begin{aligned}
& \left\{\sigma_{1}\left(p_{m}, h \exp \phi\right)-h \sigma_{1}\left(p_{m}, \exp \phi\right)\right\} \exp (-\phi) \\
& \quad=\sigma_{1}\left(p_{m}, h\right)=-i\langle\nabla h, b\rangle . \\
& \left\{\sigma_{2}\left(p_{m}, h \exp \phi\right)-h \sigma_{2}\left(p_{m}, \exp \phi\right)\right\} \exp (-\phi) \\
& \quad=\sigma_{2}\left(p_{m}, h\right)-2\left\langle\nabla h, J_{1} H_{p} J_{1} \nabla \phi\right\rangle .
\end{aligned}
$$

We get (1.5.15) from (1.4.13).

(1.5.15) $J_{1} H_{p} J_{1} \nabla \phi \equiv 2 f_{1}(A t / 2) b$,

$$
\bmod N(3,2, m-1-\varepsilon, 0)+N(1,0, m-1-\varepsilon, 0)+N(2,1, m-1,1)+N^{-\infty} \text {. }
$$

Since $\nabla h$ belongs to $N(j+1, k-1, l, \mu),(1.5 .16)$ follows.

$$
\begin{aligned}
& \left\langle\nabla h, J_{1} H_{p} J_{1} \nabla \phi\right\rangle \equiv 2\left\langle\nabla h, f_{1}(A t / 2) b\right\rangle \\
& \quad \bmod N(j+4, k+1, l+m-1-\varepsilon, \mu)+N(j+2, k-1, l+m-1-\varepsilon, \mu) \\
& \quad+N(j+3, k, l+m-1, \mu+1)+N^{-\infty}
\end{aligned}
$$

It also implies (1.5.17) that $\nabla^{2} h$ belongs to $N(j+1, k-2, l, \mu)$.

$$
\sigma_{2}\left(p_{m}, h\right) \text { belongs to } N(j+1, k-2, l+m-1, \mu) \text {. }
$$

Therefore we get (1.5.18) combining (1.5.13-17). 


$$
\begin{aligned}
& \sum_{v=1}^{2}(2 i)^{-v}(v !)^{-1}\left\{\sigma_{v}\left(p_{m}, h \exp \phi\right)-h \sigma_{v}\left(p_{m}, \exp \phi\right)\right\} \exp (-\phi) \\
& \equiv-2^{-1}\langle\nabla h, b\rangle+2^{-1}\left\langle\nabla h, f_{1}(A t / 2) b\right\rangle, \\
& \quad \bmod N(j+1, k-2, l+m-1, \mu)+N(j+4, k+1, l+m-1-\varepsilon, \mu) \\
& \quad+N(j+3, k, l+m-1, \mu+1)+N^{-\infty} .
\end{aligned}
$$

We arrive at the conclusion (1.5.12) using Proposition 1.13 and (1.5.18).

We can rewrite Lemma 1.15 to a handy type using the class $N^{*}$ to apply the induction.

Proposition 1.16. Let $g$ belong to $N^{*}(j, k, l+m-1, \mu)$. Then there exists $h$ of $N^{*}(j+1, k, l, \mu)$ that satisfies (1.5.19).

(1.5.19) $(d / d t) h+\sum_{v=1}^{2}(2 i)^{-v}(v !)^{-1}\left\{\sigma_{v}\left(p_{m}, h \exp \phi\right)\right.$

$$
\left.-h \sigma_{v}\left(p_{m}, \exp \phi\right)\right\} \exp (-\phi) \equiv g,
$$

$\bmod N^{*}(j+1, k+1, l+m-1-\varepsilon, \mu)+N^{*}(j+3, k, l+m-1, \mu+1)+N^{-\infty}$.

\section{§1.6. Induction and Estimates of the Parametrix}

It is left to show two important facts until the proof is complete. One is that $\exp \phi$ define a pseudodifferential operator belonging to $L_{0}^{0}$. The other is that $\exp \phi$ permits amplitude functions belonging to $N(j, k, l, \mu)$ at a neighborhood of the characteristic set $\Sigma$. We prepare some propositions to answer these questions.

We consider the Taylor expansion of second order for $p_{m}$. The remainder term is denoted by $g(X, Y)$. (Refer to (1.2.22) and (1.2.23).) We define $\Phi_{1}, \Phi_{2}$ and $h$ by $(1.6 .1-4)$.

$$
\begin{aligned}
& \Phi_{1}=t p_{m}+\sigma^{1}(b t / 2, F(A t / 2) b t / 2) . \\
& \Phi_{2}=p_{m}(X+\iota \chi h) t . \\
& h=h_{0}(A t / 2) b t . \\
& h_{0}(\lambda)=(2 i)^{-1} F(\lambda)\left(1+\left(\lambda^{-1} \tanh \lambda\right)^{-1 / 2}\right)^{-1}, \\
& F(\lambda)=(i \lambda)^{-1}\left(1-\lambda^{-1} \tanh \lambda\right) .
\end{aligned}
$$

Proposition 1.17. If $\gamma=1 / 2-3 \varepsilon-6 \delta>0$ and if $t$ is small, we get (1.6.5-7) on supp $\psi_{2}$.

$$
\begin{aligned}
& \Phi_{2}=\Phi_{1}+g(X, h) t \\
& |g(X, h)| \leqq C\langle\xi\rangle^{m-1-\gamma}
\end{aligned}
$$




$$
g(X, h) \text { belongs to } N(0,0, m-3 / 2+3 \varepsilon, 0) .
$$

Remark. $h$ is real valued.

Proof. At first we have to note that $h$ is real valued. Since $i b t$ is real, we have only to show that $i h_{0}(A t / 2)$ consists only of real coefficients. It follows from the fact that $h_{0}(\lambda)=-h_{0}(-\lambda)$ and $\left\{i h_{0}(\lambda)\right\}^{\operatorname{con} j}=-i h_{0}\left(\lambda^{\text {conj }}\right)$ on the domain $D$ applying Lemma 1.18. ( $z^{\text {conj }}$ means complex conjugate of $z$. Refer to Section 1.2 about the domain D.) (To be continued.)

Lemma 1.18. Let $g$ be a holomorphic function in Proposition 1.6. We assume further that ig is a real and odd function, that is, $g(\lambda)^{\mathrm{conj}}=-g\left(\lambda^{\mathrm{conj}}\right)$ and $g(\lambda)=-g(-\lambda)$. Then $g(A t / 2)$ is real, that is, the image of real vectors by $g($ At/2) are also real.

Proof of Lemma 1.18. From the definition (1.2.17) of $g(A t / 2)$ we can show that $g(A t / 2)=g(A t / 2)^{\mathrm{conj}}$ as follows because $A=-A^{\mathrm{conj}}$ and $\Gamma=-\Gamma^{\mathrm{conj}}$ taken as in the proof of Proposition 1.6.

$$
\begin{aligned}
g(A t / 2)^{\mathrm{conj}} & =\left\{(2 \pi i)^{-1} \int_{+\Gamma} g(\lambda)(\lambda-A t / 2)^{-1} d \lambda\right\}^{\mathrm{conj}} \\
& =-(2 \pi i)^{-1} \int_{-\Gamma} g\left(\lambda^{\mathrm{con} j}\right)\left(\lambda^{\mathrm{conj}}+A t / 2\right)^{-1} d \lambda^{\mathrm{conj}} \\
& =(2 \pi i)^{-1} \int_{+\Gamma} g(\mu)(\mu-A t / 2)^{-1} d \mu \\
& =g(A t / 2) .
\end{aligned}
$$

q.e.d.

Proof of Proposition 1.17, continued. Applying Proposition 1.6 to $h_{0}$, we get the estimates (1.6.9-11) because $\left|\nabla p_{m} t\right| \leqq C t\langle\xi\rangle^{m-1+\varepsilon}$ by Proposition 1.19.

(1.6.10) $h$ belongs to $N(1,1,0,0)$, which is included in $N(0,0, \varepsilon, 0)$.

$$
|h| \leqq C\left(1+t\langle\xi\rangle^{m-1}\right)^{2}\langle\xi\rangle^{\varepsilon} \leqq C\langle\xi\rangle^{2 \delta+\varepsilon} .
$$

If $2 \delta+\varepsilon<1 / 2$, then $X+\theta \iota \chi h \sim X, 0 \leqq \theta \leqq 1$, as $|\xi|$ tends to infinity because $h$ satisfies (1.6.11). This implies (1.6.12-13).

$$
\begin{gathered}
\left|\left(\nabla^{3} p_{m}\right)(X+\theta<\chi h)\right| \leqq C\langle\xi\rangle^{m-3 / 2} \\
\left|\nabla^{l}\left(\nabla^{3} p_{m}\right)(X+\theta \iota \chi h)\right| \leqq C_{l}\left(1+t\langle\xi\rangle^{m-1}\right)^{d(l)}\langle\xi\rangle^{m-3 / 2-l / 2} .
\end{gathered}
$$

By (1.2.23) $g(X, h)$ is estimated as (1.6.14-15).

$$
g(X, h) \text { belongs to } N(0,0, m-3 / 2+3 \varepsilon, 0) .
$$


(1.6.15) $|g(X, h)| \leqq C\langle\xi\rangle^{m-3 / 2+3(2 \delta+\varepsilon)}=C\langle\xi\rangle^{m-1-\gamma}$.

Since $2 \delta+\varepsilon<1 / 2$ if $\gamma>0$, we get (1.6.6-7) of Proposition 1.17 .

The Taylor expanison (1.2.22) means that we should show (1.6.16) to prove (1.6.5).

(1.6.16) $\sigma^{1}(b t / 2, F(\mathrm{~A} t / 2) b t / 2)=\left\langle\nabla p_{m}, h\right\rangle t+\left\langle\nabla^{2} p_{m} h, h\right\rangle t / 2$.

In fact the right hand side of (1.6.16) is rewritten as (1.6.17) by the definition of $h$.

$$
\begin{aligned}
& \left\langle\nabla p_{m} t, h\right\rangle+\left\langle\nabla^{2} p_{m} t h / 2, h\right\rangle \\
& \quad=i\left\{\sigma^{1}(b t, h)+\sigma^{1}((A t / 2) h, h)\right\} \\
& \quad=i\left\{\sigma^{1}\left(b t, h_{0}(A t / 2) b t\right)+\sigma^{1}\left((A t / 2) h_{0}(A t / 2) b t, h_{0}(A t / 2) b t\right)\right\} \\
& \quad=i \sigma^{1}\left(b t,\left\{h_{0}(A t / 2)+(A t / 2) h_{0}(A t / 2)^{2}\right\} b t\right),
\end{aligned}
$$

because $\sigma^{1}\left(u, J_{1} f\right)=\langle u, f\rangle$ and $h_{0}(\lambda)$ is an odd function in $\lambda$. The last term of (1.6.17) is equal to $\sigma^{1}(b t / 2, F(A t / 2) b t / 2)$ the left hand side of (1.6.16) because $h_{0}(\lambda)+\lambda h_{0}(\lambda)^{2}=-i F(\lambda) / 4$.

q.e.d.

The other propositions are also based on the following simple and important proposition which gets our proof into shape under Condition (A).

Proposition 1.19. The principal symbol $p_{m}$, which is non-negative, satisfies (1.6.18) for some constant $C$,

$$
\left|\nabla p_{m}\right|^{2} \leqq C p_{m}\langle\xi\rangle^{m-1} .
$$

Proof. The proof is a direct application of Lemma 1.20 which is a well known result for a non-negative $C^{2}$-function with a compact support. q.e.d.

Lemma 1.20. Let $f$ be a real valued and non-negative $C^{2}$-function with a compact support on $\mathbb{R}^{n}$. Then $f$ satisfies (1.6.19), where $H_{f}(x)$ is the Hesse matrix of $f$.

$$
|\operatorname{grad} f(x)|^{2} \leqq 2 f(x) \sup _{y \in \mathbf{R}^{n}}\left\|H_{f}(y)\right\| .
$$

We omit the proof.

Proposition 1.21. There exist consianis c and $d$ such that

$$
\left|\nabla \Phi_{2}\right|^{2} \leqq c \Phi_{2} t\langle\zeta\rangle^{m-1}\left(1+r\langle\xi\rangle^{m-1}\right)^{d} .
$$

Proof. By definition, $\nabla \Phi_{2}$ is written as

$$
\nabla \Phi_{2}=R \nabla p_{m}(X+\iota \chi h) t
$$

where 


$$
\begin{aligned}
& R=\left(R_{i j}\right)_{i, j=1,2}, \quad R_{11}=\langle\xi\rangle^{-1 / 2}\langle\eta\rangle^{1 / 2} \partial_{x} y, \\
& R_{12}=\langle\xi\rangle^{-1 / 2}\langle\eta\rangle^{-1 / 2} \partial_{x} \eta, \quad R_{21}=\langle\xi\rangle^{1 / 2}\langle\eta\rangle^{1 / 2} \partial_{\xi} y, \\
& R_{22}=\langle\xi\rangle^{1 / 2}\langle\eta\rangle^{-1 / 2} \partial_{\xi} \eta, \text { and }(y, \eta)=X+\measuredangle \chi h .
\end{aligned}
$$

$\left(\nabla p_{m}\right)(X+\iota \chi h)$ is estimated as (1.6.22) by applying Proposition 1.19, because $\langle\xi\rangle \sim\langle\eta\rangle$ as we noted in the proof of Proposition 1.17.

$$
\left|\left(\nabla p_{m}\right)(X+\iota \chi h) t\right|^{2} \leqq C \Phi_{2} t\langle\xi\rangle^{m-1} .
$$

On the other hand we get (1.6.23) noting that $R_{i 1}=\langle\eta\rangle^{1 / 2} \nabla y$ and $R_{i 2}=\langle\eta\rangle^{-1 / 2} \nabla \eta,(i=1,2)$, because of (1.6.10), (1.3.33) and (1.3.4).

$$
\left|R_{i j}\right| \leqq C\left(1+t\langle\xi\rangle^{m-1}\right)^{d} .
$$

(1.6.22-23) imply (1.6.20).

q.e.d.

\section{Lemma 1.22.}

$$
b t\left(=i J_{1} \nabla p_{m} t\right)=-i\left\{f_{0}(A t / 2)\right\}^{-1} J_{1} \nabla \Phi_{2}+\beta,
$$

where $\beta$ belongs to $N(1,0,2 \varepsilon-1 / 2,0)$ and $f_{0}(\lambda)=\lambda^{-1} \tanh \lambda$.

Proof. By (1.4.10),

(1.6.25) $i J_{1} \nabla \Phi_{1} \equiv-f_{0}(A t / 2) b t, \quad \bmod \quad N(3,2,-1 / 2,0)+N(1,0,-1 / 2,0)$.

So we get (1.6.26) since $f_{0}(A t / 2)$ is invertible.

(1.6.26) $b t \equiv-\left\{f_{0}(A t / 2)\right\}^{-1} i J_{1} \nabla \Phi_{1}, \bmod N(3,2,-1 / 2,0)+N(1,0,-1 / 2,0)$.

(1.6.27) holds by Proposition 1.17.

$$
\nabla \Phi_{1} \equiv \nabla \Phi_{2}, \quad \bmod \quad N(1,0,2 \varepsilon-1 / 2,0) .
$$

Combining (1.6.26-27) we get (1.6.24) because $N(3,2,-1 / 2,0)$ and $N(1,0,-1 / 2,0)$ are included in $N(1,0,2 \varepsilon-1 / 2,0)$.

q.e.d.

Proposition 1.23. If $6 \varepsilon \leqq 1$, then there exist constants $c$ and $d$ such that

$$
|b t|^{2} \leqq c t\langle\xi\rangle^{m-1}\left(\Phi_{2}+t\langle\xi\rangle^{m-1-1 / 3}\right)\left(1+t\langle\xi\rangle^{m-1}\right)^{d} .
$$

Proof. We use (1.6.24) of Lemma 1.22. Since $\beta$ belongs to $N(1,0,2 \varepsilon$ $-1 / 2,0), \beta$ is bounded by $t\langle\xi\rangle^{m-1-1 / 6}\left(1+t\langle\xi\rangle^{m-1}\right)^{d}$. For $\nabla \Phi_{2}$ there is (1.6.20) of Proposition 1.21.

q.e.d.

Now we estimate $\exp \phi$, which is a product of three parts (1.6.29-31). (Refer to $(0.2 .6)$ for $\phi$.)

$$
\exp \left\{\left(1-\psi_{1}\right) \phi_{2}\right\} .
$$




$$
\begin{gathered}
\exp \left\{-\psi_{1} \Phi_{1}\right\} . \quad(\operatorname{Refer} \text { to (1.6.1).) } \\
\exp \left\{-p_{m-1} t\right\}[\operatorname{det}\{\cosh (A t / 2)\}]^{-\psi_{1} / 2} .
\end{gathered}
$$

Lemma 1.24. If $t$ is small and if $\gamma=1 / 2-3 \varepsilon-6 \delta$, we get (1.6.32-33).

$$
\begin{aligned}
& \left|\exp \left\{-\psi_{1} \Phi_{1}\right\}\right| \leqq \exp \left\{-\psi_{1} \Phi_{2}\right\} \exp \left\{c \psi_{1}\langle\xi\rangle^{m-1-\gamma} t\right\} \\
& |\operatorname{det}\{\cosh (A t / 2)\}| \geqq 2^{-2 n} \exp \left\{\operatorname{Tr}^{\sim} A t\right\}, \quad \text { on } \operatorname{supp} \psi_{1} .
\end{aligned}
$$

Proof. (1.6.32) is immediately proved by (1.6.5). If the eigenvalues of At $/ 2$ are denoted by $\lambda_{j}(j=1, \ldots, 2 n),\left\{\lambda_{j}\right\}$ lie on the zonal domain $Z=\{\lambda ;|\operatorname{Im} \lambda|$ $<1 / 2\}$. (Refer to Lemma 1.7.) And also the eigenvalues of $\cosh (A t / 2)$ are $\cosh \lambda_{j}$, which satisfy (1.6.34).

$$
\begin{aligned}
\left|\cosh \lambda_{j}\right| & =2^{-1} \exp \left(-\operatorname{Re} \lambda_{j}\right)\left|1+\exp \left(2 \lambda_{j}\right)\right| \\
& =2^{-1} \exp \left(\operatorname{Re} \lambda_{j}\right)\left|1+\exp \left(-2 \lambda_{j}\right)\right| .
\end{aligned}
$$

$\operatorname{Re} \exp \left(-2 \lambda_{j}\right) \geqq 0$ and $\operatorname{Re} \exp \left(2 \lambda_{j}\right) \geqq 0$ because $\left|\operatorname{Im} 2 \lambda_{j}\right|<\pi / 2$. These imply that $\left|\cosh \lambda_{j}\right| \geqq 2^{-1} \exp \left(\left|\operatorname{Re} \lambda_{j}\right|\right)$. Therefore we get (1.6.35) because $\lambda$ is an eigenvalue of $A t / 2$ if $-\lambda$ is so.

$$
\begin{aligned}
& |\operatorname{det}\{\cosh (A t / 2)\}|=\prod_{j=1}^{2 n}\left|\cosh \lambda_{j}\right| \\
& \quad \geqq 2^{-2 n} \exp \left(\sum_{j=1}^{2 n}\left|\operatorname{Re} \lambda_{j}\right|\right)=2^{-2 n} \exp \left(\operatorname{Tr}^{\sim} A t\right) . \quad \text { q.e.d. }
\end{aligned}
$$

Proposition 1.25. There exist positive constants $c$ and $c_{0}$ such that $\exp \phi$ is estimated as (1.6.36) if $t$ is small, where $\phi_{0}$ is defined in (1.6.37). (Refer to $(0.2 .7)$ for $\psi_{1}^{1}$.)

$$
\begin{gathered}
|\exp \phi| \leqq c_{0} \exp \left(-\phi_{0}\right) . \\
\phi_{0}=\Phi_{2} \psi_{1}+p_{m} t\left(1-\psi_{1}^{1}\right)+c t\langle\xi\rangle^{m-1} .
\end{gathered}
$$

Proof. Since $\exp \phi$ is a product of (1.6.29-31), it is estimated as (1.6.38) using Lemma 1.24.

(1.6.38) $|\exp \phi|$

$$
\leqq c_{1} \exp \left\{\left(1-\psi_{1}\right) \phi_{2}-\psi_{1}\left(\Phi_{2}-c_{2}\langle\xi\rangle^{m-1-\gamma} t+\operatorname{Re} p_{m-1} t+2^{-1} \operatorname{Tr}^{\sim} A t\right)\right\} .
$$

By Condition (A), $\operatorname{Re} p_{m-1} t+2^{-1} \operatorname{Tr}^{\sim} A t \geqq c_{3} t\langle\xi\rangle^{m-1}$ for a positive constant $c_{3}$. Since $\gamma>0$ and $t$ is bounded, we get (1.6.39) with positive constants $c_{4}$ and $c_{5}$.

$$
\begin{aligned}
& -\psi_{1}\left(-c_{2} t\langle\xi\rangle^{m-1-\gamma}+\operatorname{Re} p_{m-1} t+2^{-1} \operatorname{Tr} \sim A t\right) \\
& \leqq-\psi_{1} c_{4} t\langle\zeta\rangle^{m-1}+c_{5} .
\end{aligned}
$$

On the other hand $\left(1-\psi_{1}\right) \phi_{2}$ satisfies (1.6.40). 


$$
\begin{aligned}
\left(1-\psi_{1}\right) \phi_{2} & =-\left(1-\psi_{1}^{1}\right) p_{m} t-\psi_{1}^{1}\left(1-\psi_{1}^{2}\right) p_{m} t-\left(1-\psi_{1}\right) t\langle\xi\rangle^{m-1} \\
& \leqq-\left(1-\psi_{1}^{1}\right) p_{m} t-\left(1-\psi_{1}\right) t\langle\xi\rangle^{m-1}
\end{aligned}
$$

Thus, we get the estimates (1.6.36) for $|\exp \phi|$ putting $c_{0}=c_{1} \exp c_{5}$ and $c=2^{-1} \min \left\{c_{4}, 1\right\}$.

q.e.d.

Products of $\exp \phi$ and elements of $N_{g}(j, k, l, \mu)$ appear in the parametrix and its derivatives. The following Proposition 1.26 acts effectively on their estimations with Proposition 1.25.

Proposition 1.26. Let $f$ be an element of $N_{g}(j, k, l, \mu),(\mu<+\infty)$. We assume that $j \geqq k \geqq 0$ and we put $v=\mu \theta(0 \leqq \theta<1)$. Then, we get the estimate (1.6.41) with constants $c$, $d$ and $\kappa(v)=\kappa(v, j, k, l)$, where $\kappa(v)=l-2 \varepsilon v$ if $j-k>v, l-\varepsilon(j-k+v)$ if $j-k \leqq v$ and if $v<j$, or $l-\varepsilon(2 j-k)$ if $v \geqq j$.

$$
|f| \leqq c\left(1+\phi_{0}\right)^{d}\langle\xi\rangle^{\kappa(v)} .
$$

(Refer to (1.6.37) for $\phi_{0}$.)

Proof. By definition $f$ is written as (1.6.42), where $\left(1-\psi_{1}^{1}\right)^{v}=1$ if $v=0$, which is estimated as (1.6.43). (Refer to Section 1.3.)

$$
\begin{gathered}
f=\left(t\langle\xi\rangle^{m-1}\right)^{j-k} g(t b)\left(1-\psi_{1}^{1}\right)^{v} . \\
|f| \leqq c\left(1+t\langle\xi\rangle^{m-1}\right)^{d}\left(t\langle\xi\rangle^{m-1}\right)^{j-k}|t b|^{k}\langle\xi\rangle^{l}\left(1-\psi_{1}^{1}\right)^{v} .
\end{gathered}
$$

We know that $t\langle\xi\rangle^{m-1} \leqq c t p_{m}\langle\xi\rangle^{-2 \varepsilon}$ and $|t b| \leqq c t p_{m}\langle\xi\rangle^{-\varepsilon}$ on $\operatorname{supp}\left(1-\psi_{1}^{1}\right)$. These imply (1.6.44-46).

(1.6.44) If $j-k>v$,

$$
|f| \leqq c\left(1+t\langle\xi\rangle^{m-1}\right)^{d}\left(t\langle\xi\rangle^{m-1}\right)^{j-k-v}|t b|^{k}\langle\xi\rangle^{l-2 \varepsilon v}\left\{t p_{m}\left(1-\psi_{1}^{1}\right)\right\}^{v} .
$$

(1.6.45) If $j-k \leqq v$ and $j>v$,

$$
|f| \leqq c\left(1+t\langle\xi\rangle^{m-1}\right)^{d}|t b|^{j-v}\langle\xi\rangle^{l-\varepsilon(j-k+v)}\left\{t p_{m}\left(1-\psi_{1}^{1}\right)\right\}^{v} .
$$

(1.6.46) If $j \leqq v$,

$$
|f| \leqq c\left(1+t\langle\zeta\rangle^{m-1}\right)^{d}\langle\xi\rangle^{l-\varepsilon(2 j-k)}\left\{t p_{m}\left(1-\psi_{1}^{1}\right)\right\}^{j} .
$$

When $j>v$, we further estimate them by using (1.6.47) for some $\alpha \geqq 0$.

$$
|f| \leqq c\left(1-\psi_{1}^{1}\right)^{\alpha}|f|+\mathrm{c}\left(\psi_{1}\right)^{\alpha}|f|+g, \quad g \in N_{g}^{-\infty} .
$$

Since $\left(t\langle\zeta\rangle^{m-1}\right)^{\beta}|t b|^{\gamma} \leqq c\left|t p_{m}\right|^{\beta+\gamma}$ on $\operatorname{supp}\left(1-\psi_{1}^{1}\right)$, we get (1.6.48) if $\alpha \geqq j-v$.

$$
\left(1-\psi_{1}^{1}\right)^{\alpha}|f| \leqq c\left(1+t\langle\xi\rangle^{m-1}\right)^{d}\left\{t p_{m}\left(1-\psi_{1}^{1}\right)\right\}^{j}\langle\xi\rangle^{\kappa(v)} .
$$

On the other hand, we know Proposition 1.23. So we get (1.6.49) with another constant $d$ if $\alpha \geqq j-v$. 
(1.6.49) $\left(\psi_{1}\right)^{\alpha}|f|$

$$
\leqq c\left(1+t\langle\zeta\rangle^{m-1}\right)^{d}\left\{\psi_{1}\left(\Phi_{2}+t\langle\zeta\rangle^{m-1}\right)\right\}^{j-k}\left\{t p_{m}\left(1-\psi_{1}^{1}\right)\right\}^{v}\langle\xi\rangle^{\kappa(v)} .
$$

Since the remainder term $g$ of $N_{g}^{-\infty}$ is estimated as (1.4.50), we get (1.6.41) rewriting $d+j+v$ to $d$ and noting (1.6.37).

$$
|g| \leqq c\left(1+t\langle\xi\rangle^{m-1}\right)^{d}\langle\xi\rangle^{-\beta},
$$

for any $\beta$ and for some constants $c$ and $d$ depending on $\beta$ because $21\langle\xi\rangle^{m-1}$ $\geqq\langle\xi\rangle^{\delta}$ on suppg. (Refer to (1.3.25) and the remark at the end of Scction 1.3 for $N_{g}^{-\infty}$.)

q.e.d.

Proposition 1.27. Let $f$ be an element of $N_{g}^{* *}(j, k, l, \mu)$. We put $\nabla^{\alpha}(f \exp \phi)=g_{\alpha} \exp \phi,(\alpha \geqq 0)$. Then $g_{\alpha}$ also belongs to $N_{g}^{* *}(j, k, l, \mu)+N_{g}^{-\infty}$. (Refer to (1.3.54) for $N_{g}^{* *}$.)

Proof. By (1.4.6), $(1,4,10)$ and (1.3.48) $\nabla \phi$ belongs to $N_{g}^{*}(1,1,0,0)$ $+N_{g}^{-\infty}$. Therefore $\nabla f+f \nabla \phi$ also belongs to $N_{g}^{* * *}(j, k, l, \mu)+N_{g}^{-\infty}$ by (1.3.47) and the definition of $N_{g}^{* *}$. This fact implies inductively the conclusion.

q.e.d.

Proposition 1.28. Let $f$ be an element of $N_{g}^{* *}(j, k, l, \mu), \quad(j \geqq k \geqq 0)$. Then $f \exp \phi$ belongs to $L_{0}^{\kappa(v)}$ if $0 \leqq t \leqq T$, where $v$ and $\kappa(v)$ are those defined in Proposition 1.26 and $T$ is a small positive constant which is independent of $f$. Especially $\exp \phi$ belongs to $L_{0}^{0}$. More strictly $\nabla^{j} f \exp \phi$ are bounded by $c_{j}\langle\xi\rangle^{\kappa(v)} \exp \left(-\phi_{0} / 2\right)$. (Refer to (1.3.53) for $N_{g}^{* *}$ and to the remark after Theorem 0.1 for $L_{\rho}^{m}$.)

Proof. By Proposition 1.27 we have only to show that $f \exp \phi$ is bounded by $c\langle\xi\rangle^{\kappa(v)} \exp \left(-\phi_{0} / 2\right) . \quad f$ is a finite sum of elements of $N_{g}\left(j^{\prime}, k^{\prime}, l, \mu\right)$ such that $j^{\prime} \geqq j$ and $j^{\prime}-k^{\prime} \geqq j-k$. Combining Proposition 1.25 and 1.26, $f \exp \phi$ with respect to such $f$ belonging to $N_{g}\left(j^{\prime}, k^{\prime}, l, \mu\right)$ is bounded by $c\langle\xi\rangle^{\kappa\left(v, j^{\prime}, k^{\prime}, l\right)}$ $\exp \left(-\phi_{0} / 2\right)$ if we take $T$ as Proposition 1.25 holds. It is also bounded by $c\langle\xi\rangle^{\kappa(v, j, k, l)} \exp \left(-\phi_{0} / 2\right)$ because $\kappa(v, j, k, l) \geqq \kappa\left(v, j^{\prime}, k^{\prime}, l^{\prime}\right)$ if $j^{\prime} \geqq j, j^{\prime}-k^{\prime}$ $\geqq j-k$ and $l^{\prime} \leqq l$.

q.e.d.

We have been ready for a construction and estimates of a parametrix except for how to define amplitudes outside of the characteristic set. However it is easy as we noted at the introduction and it is written as follows.

Proposition 1.29. Let $g_{0}$ be an element of $N_{g}(j, k, l+m-1$, out $)$. Then $f=\int_{0}^{t} g_{0} d t$ belongs to $N_{g}(j+1, k, l$, out $)$ and satisfies (1.6.51) for $\alpha \geqq 0$, where 
$g_{1}$ belongs to $N_{g}(j+1, k, l+m-1$, out $)+N_{g}(j+1, k+1, l+m-1-\varepsilon$, out $)$ $+N_{g}^{-\infty}$.

$(1.6 .51)(d / d t)(f \exp \phi)+\sum_{v=0}^{\alpha}(2 i)^{-v}(v !)^{-1} \sigma_{v}(p, f \exp \phi)$

$$
=\left(g_{0}+g_{1}\right) \exp \phi \text {. }
$$

(Refer to the remark at the end of Section 1.3 for $N_{g}(j, k, l$, out).)

Proof. By definition it is clear that $f$ belongs to $N_{g}(j+1, k, l$, out $)$ and satisfies (1.6.52) because $\phi=-p_{m} t-t\langle\xi\rangle^{m-1}$ on $\operatorname{supp} f$.

$$
\begin{aligned}
& (d / d t)(f \exp \phi)+p_{m} f \exp \phi+(2 i)^{-1} \sigma_{1}\left(p_{m}, f \exp \phi\right) \\
& \quad=\left\{g_{0}-\langle\xi\rangle^{m-1} f+(2 i)^{-1}\left(\sigma_{1}\left(p_{m}, f\right)-\sigma_{1}\left(p_{m},\langle\xi\rangle^{m-1}\right) t f\right)\right\} \exp \phi .
\end{aligned}
$$

The terms at the right hand side except for $g_{0}$ clearly belong to $N_{g}(j+1, k, l$ $+m-1$, out $)+N_{g}(j+1, k+1, l+m-1-\varepsilon$, out $)$. On the other hand, if we put $\sigma_{v}(p, f \exp \phi)=g(v) \exp \phi$ and if $v \geqq 2, g(v)$ belongs to $N_{g}(j+1, k, l+m-1$, out $)$ $+N_{g}^{-\infty}$ by Proposition 1.27. So we get the conclusion. q.e.d.

We extend Propositions 1.12 and 1.16 to global ones combining Proposition 1.29 and them.

\section{Proposition 1.30.}

$$
\begin{aligned}
& (d / d t) \phi+p_{m}+p_{m-1}+\sum_{v=1}^{2}(2 i)^{-v}(v !)^{-1} \sigma_{v}\left(p_{m}, \exp \phi\right) \exp (-\phi) \\
& \quad \equiv 0, \quad \bmod N_{g}^{* *}(1,1, m-1-\varepsilon, 0)+N_{g}^{* *}(0,0, m-1,1)+N_{g}^{-\infty} .
\end{aligned}
$$

Proof. We denote the left hand side of (1.6.53) by $g_{0} . g_{0}$ is written as a sum of two parts $\psi_{2} g_{0}$ and $\left(1-\psi_{2}\right) g_{0}$. Since $g_{0}$ satisfies (1.4.2) on supp $\psi_{2}$ by Proposition 1.12, $\psi_{2} g_{0}$ belongs to $N_{g}^{* *}(1,1, m-1-\varepsilon, 0)+N_{g}^{* *}(0,0, m-1,1)$ $+N_{g}^{-\infty}$. Since $\phi=-p_{m} t-t\langle\xi\rangle^{m-1}$ on $\operatorname{supp}\left(1-\psi_{2}\right), g_{0}$ belongs to $N_{g}(0,0, m$ $-1,0)+N_{g}(1,1, m-1,0)+N_{g}(2,2, m-1,0)+N_{g}^{-\infty}$ there. So $\left(1-\psi_{2}\right) g_{0}$ belongs to $N_{g}^{* * *}(0,0, m-1$, out $)+N_{g}^{-\infty}$.

q.e.d.

Proposition 1.31. Let $g$ belong to $N_{g}^{*}(j, k, l+m-1, \mu),(j \geqq k)$. Then there exists $h$ belonging to $N_{g}^{*}(j+1, k, l, \mu)$ and satisfying (1.6.54).

$$
\begin{gathered}
(d / d t) h+\sum_{v=1}^{2}(2 i)^{-v}(v !)^{-1}\left\{\sigma_{v}\left(p_{m}, h \exp \phi\right)-h \sigma_{v}\left(p_{m}, \exp \phi\right)\right\} \exp (-\phi) \equiv g, \\
\bmod N_{g}^{*}(j+1, k+1, l+m-1-\varepsilon, \mu)+N_{g}^{*}(j+1, k, l+m-1, \mu+1)+N_{g}^{-\infty} .
\end{gathered}
$$

Proof. By Proposition 1.16 there exists $h_{1}$ belonging to $N^{*}(j+1, k, l, \mu)$ and satisfying (1.5.19). We consider $\psi_{2} h_{1}$, which belongs to $N_{g}^{*}(j+1, k, l, \mu)$ and satisfies (1.6.54) replaced $g$ by $\psi_{2} g$. In fact $\sigma\left(\nabla p_{m}, \nabla \psi_{2}\right) h, \sigma\left(\nabla^{2} p_{m}\right.$, 
$\left.\nabla \psi_{2} \nabla \phi\right) h$ and $\sigma\left(\nabla^{2} p_{m}, 2 \nabla \psi_{2} \nabla h+h \nabla^{2} \psi_{2}\right)$ belong to $N_{g}^{*}(j+1, k+1, l+m-1$ $-\varepsilon, \mu)$ because $\psi_{2}$ and $\nabla \phi$ belong to $N_{g}(0,0,0,0)$ and $N_{g}^{*}(1,1,0,0)+N_{g}^{-\infty}$, respectively. For $\left(1-\psi_{2}\right) g$, which belongs to $N_{g}^{*}(j, k, l+m-1$, out $)+N_{g}^{-\infty}$, there exists $h_{2}$ which belongs to $N_{g}^{*}(j+1, k, l, \mu)$ and satisfies (1.6.51) $\bmod N_{g}^{*}(j$ $+1, k+1, l+m-1-\varepsilon$, out $)+N_{g}^{*}(j+1, k, l+m-1$, out $)$. Therefore, putting $h=h_{1}+h_{2}$, we get $h$ which belongs to $N_{g}^{*}(j+1, k, l, \mu)$ and satisfies (1.6.54).

q.e.d.

To state briefly the conclusion of this chapter we introduce two more classes $M(l)$ and $M^{*}(l)$ defined by (1.6.55-58).

(1.6.55) $M(1)=\sum_{j+k=1} N_{g}^{* *}(j+1, j,-k \varepsilon, j)+N_{g}^{* *}(1,0,0,1)$.

(1.6.56) $M(l)=\sum_{j+k=l} N_{g}^{* *}(j+1, j,-k \varepsilon, j), \quad$ if $l>1$.

(1.6.57) $\quad M^{*}(1)=\sum_{j+k=1} N_{g}^{* *}(j, j, m-1-k \varepsilon, j)+N_{g}^{* *}(0,0, m-1,1)+N_{g}^{-\infty}$.

(1.6.58) $\quad M^{*}(l)=\sum_{j+k=l} N_{g}^{* *}(j, j, m-1-k \varepsilon, j)+N_{g}^{-\alpha}, \quad$ if $l>1$.

Theorem 1.1. Let $\phi$ be defined by $(0.2 .3-8)$. Then there exist $f_{j}$ belonging to $M(j)(j=1,2, \ldots)$ such that, if we put

$$
E_{n}=\sum_{j=0}^{n} f_{j} \exp \phi
$$

and

$$
G_{n}=g_{n} \exp \phi=\left(\partial_{t}+p\right)_{(n+2)} E_{n},
$$

then $g_{n}$ belongs to $M^{*}(n+1)$, where $f_{0}=1$. Thus $f_{i} \exp \phi$ belongs to $L_{0}^{-\varepsilon j}$ and $G_{n}$ belongs to $L_{0}^{m-1-\varepsilon(n+1)}(n \geqq 1)$.

Remark. ${ }^{\circ}(n+2)$ means $(n+2)$-th sum of the asymptotic expansion of the product of two pseudodifferential operators $\left(\partial_{t}+p\right)$ and $E_{n}$, that is,

$$
\left(\partial_{t}+p\right)_{(n+2)} E_{n}=\partial_{t} E_{n}+\sum_{k=0}^{n+2}(2 i)^{-k}(k !)^{-1} \sigma_{k}\left(p, E_{n}\right) .
$$

Proof. By Proposition $1.30 g_{0}$ belongs to $M^{*}(1)$. We denote the part of $g_{0}$ belonging to $N_{g}^{* * *}(0,0, m-1,1)$ by $g_{0}^{\prime}$. By Proposition 1.31 there exists $f_{1}^{\prime}$ of $N_{g}^{* *}(1,0,0,1)$ satisfying (1.6.54) with respect to $-g_{0}^{\prime}$. We consider (1.6.60) for $\left(1+f_{1}^{\prime}\right) \exp \phi$ and for $n=0$, and denote the remainder term by $g_{1}^{\prime \prime} \exp \phi . \quad g_{1}^{\prime \prime}$ belongs to $\sum_{j+k=1} N_{g}^{* *}(j, j, m-1-k \varepsilon, j)+N_{g}^{-\infty}$. We apply again Proposition 1.31 to $-g_{1}^{\prime \prime}$ we get $f_{1}^{\prime \prime}$ belonging to $\sum_{j+k=1} N_{g}^{* *}$ $(j+1, j,-k \varepsilon, j)$. We put $f_{1}=f_{1}^{\prime}+f_{1}^{\prime \prime}$. Then $f_{1}$ belongs to $M(1)$ and $g_{1}^{\prime}$ in the remainder term $g_{1}^{\prime} \exp \phi$ of (1.6.60) for $\left(f_{0}+f_{1}\right) \exp \phi$ and for $n=0$ belongs to $M^{*}(2)$. By Proposition $1.27 \sigma_{3}\left(p,\left(f_{0}+f_{1}\right) \exp \phi\right) \exp (-\phi)$ also belongs to 
$M^{*}(2)$. Thus there exists $f_{1}$ such that (1.6.60) holds when $n=1$. In general we assume that (1.6.60) holds in the case $n$. At first we note that $\sigma_{n+3}\left(p, E_{n+1}\right)$ $\times \exp (-\phi)$ and $\sigma_{k}\left(p, f_{n+1} \exp \phi\right) \exp (-\phi)(k \geqq 3)$ belong to $M^{*}(n+2)$ if $f_{n+1}$ exists. We apply again Proposition 1.31 to $-g_{n}$, and we denote its solution by $f_{n+1}$, which clearly belongs to $M(n+1)$. If we consider (1.6.60) for $n+1$, the term $g_{n+1}$ belongs to $M^{*}(n+2)$. The estimate for $E_{n}$ and $G_{n}$ are obtained by applying Proposition 1.28 in the case that $v=j / 2$ and by noting that $f \exp \phi$ with respect to $f$ of $N_{g}^{-\infty}$ belongs to $S^{-\infty}$.

q.e.d.

\section{Chapter 2. Representation of the Fundamental Solution by Pseudodifferential Operators}

Since the parametrix obtained by Theorem 1.1 at the previous chapter was a pseudodifferential operator in the class $L_{0}^{0}$, it will be natural to consider about representation of the fundamental solution by pseudodifferential operators. The discussion at this chapter is simple if we assumed a proposition for powers of pseudodifferential operators mentioned at the first section of this chapter.

\section{§2.1. Fundamental Solution}

We will solve a Volterra's integral equation including pseudodifferential operators. It is well known for the usual Volterra's integral equation to be solved by a successive approximation. We also do it, while we have to use estimates of symbols for powers of pseudodifferential operators when we estimate powers of integral operators and prove convergences of asymptotic series. The following proposition guarantees them.

Proposition 2.1. Let $p_{j}(j=1, \ldots, v)$ be in $L_{0}^{m(j)}$. Then $p=p_{1} \circ \cdots \circ p_{v}$ is in $L_{0}^{m(0)}\left(m(0)=\sum_{j=1}^{v} m(j)\right)$ and satisfies (2.1.1) for all integer $l \geqq 0$ and for some integer $l_{0}$ and constant $c_{l}$ which are dependent on $l$ and $\sum_{j=1}^{v}|m(j)|$ but independent of $v$.

$$
|p|_{i}^{(m(0))} \leqq\left(c_{l}\right)^{v} \prod_{j=1}^{\prime}\left|p_{j}\right|_{l_{0}}^{(m(j))},
$$

where $|p|_{l}^{(m)}=\max _{k \leqq l}\left\{\sup _{(x, \xi) \in \mathbf{R}^{2 n}}\left|\nabla^{k} p(x, \xi)\right|\langle\xi\rangle^{-m}\right\}$.

(We shall prove it at Appendix. And also refer to C. Iwasaki [7].)

Let $E(t)$ be the fundamental solution of $(0.0 .1)$, that is, the solution of (2.1.2). At first we should assume its unique existence in a suitable sense. 
Then, $E(t)$ would satisfy an integral equation (2.1.3). Conversely if $E(t)$ were a unique solution of (2.1.3) would be also one of (2.1.2). So we have only to solve (2.1.3) to show the existence of a fundamental solution, where $E_{n}(t)$ is an $n$-th partial sum of the parametrix for (2.1.2) defined at Theorem 1.1 and $G_{n}$ is its error term.

$$
\begin{aligned}
& ((d / d t)+P) E(t)=0 \\
& E(0)=I \\
& E(t)+\int_{0}^{t} E(t-s) G_{n}(s) d s=E_{n}(t) \\
& E(0)=I
\end{aligned}
$$

where $E_{n}(t)=e_{n}(t, x, D), e_{n}=\sum_{j=0}^{n} f_{j} \exp \phi$ and $G_{n}(t)=((d / d t)+P) E_{n}(t)$.

Lemma 2.2. $E_{n}(t)$ and $G_{n}(t)$ are pseudlodifferential operators belonging to $L_{0}^{0}$ and $L_{0}^{\kappa}, \kappa=m-1-\varepsilon(n+1)$, and their derivatives in $t(d / d t)^{j} E_{n}(t)$ and $(d / d t)^{j} G_{n}(t)$ belong to $L_{0}^{m j}$ and $L_{0}^{\kappa+m j}$, respectively. Moreover they belong to $S^{-\infty}$ if $t>0$.

Proof. It is the result of Theorem 1.1 that $(d / d t)^{j} E_{n}(t)$ belongs to $L_{0}^{m j}$, and $S^{-\infty}$ if $t>0$. By the expansion formula (Proposition 1.1) there exists $l$ such that $(d / d t)^{j} G_{n}-((d / d t)+P)^{\circ}{ }_{(l)}(d / d t)^{j} E_{n}$ belongs to $L^{\kappa+m j}$. Theorem 1.1 means that $((d / d t)+P)^{\circ}{ }_{(n+1)}(d / d t)^{j} E_{n}$ belongs to $L^{\kappa+m j}$. Since it is clear that $\sigma_{k}\left(p,(d / d t)^{j} e_{n}\right)$ belongs to $L^{\kappa+m j}$ if $k>n+1$ and that $G_{n}(t)$ belongs to $S^{-\infty}$ if $t>0$, we get the conclusion.

q.e.d.

Theorem 2.1. There exists a pseudodifferential operator $H_{n}(t)$ belonging to $L_{0}^{\kappa}, \kappa=m-1-\varepsilon(n+1)$ if $\kappa<0$, and to $S^{-\infty}$ if $t>0$, such that

$$
E(t)=E_{n}(t)-\int_{0}^{t} E_{n}(s) H_{n}(t-s) d s
$$

is the unique solution of (2.1.3) and belongs to $L_{0}^{0}$, that is, $E(t)$ is the unique solution of (2.1.2), where the uniqueness holds as operators from $\mathscr{S}$ to $\mathscr{S}^{\prime}$ which satisfy (2.1.2) in weak sense.

Remark. The adjoint equation $\left((d / d t)-P^{*}\right) u=0$ has a fundamental solution given by $E(-t)^{*}$ in the negative direction of $t$. And also we note that $(d / d t)^{j} E(t)$ and $(d / d t)^{j} H_{n}(t)$ belong to $L_{0}^{m j}$ and $L_{0}^{\kappa+m j}$, respectively.

Proof. $H_{n}(t)$ will be given by the following asymptotic series (2.1.5), where $K_{j}(t)$ are defined inductively by (2.1.6). 


$$
\begin{aligned}
& H_{n}(t)=\sum_{j=0}^{+\infty} K_{j}(t) . \\
& K_{0}(t)=G_{n}(t), \\
& K_{j}(t)=-\int_{0}^{t} G_{n}(s) K_{j-1}(t-s) d s .
\end{aligned}
$$

If we denote the symbols of $K_{j}(t)$ and $G_{n}(t)$ by $k_{j}(t)$ and $g_{n}(t), k_{j}(t)$ is estimated as (2.1.7) by Proposition 2.1.

$$
\left|k_{j}(t)\right|_{l}^{(j \kappa)} \leqq(j !)^{-1}\left(c_{l} t\right)^{j}\left(A_{l}\right)^{j+1},
$$

where $A_{l}=\sup _{0<t<T}\left|g_{n}(t)\right|_{i}^{(\kappa)}$. This implies that $\sum_{j=0}^{+\infty} k_{j}(t)$ converges in $L_{0}^{\kappa}$ if $\kappa<0$. We put it $h_{n}(t)$ so that $H_{n}(t)$ belongs to $L_{0}^{\kappa}$. We can show that $K_{j}(t)$ belongs to $S^{-\infty}$ if $t>0$ because $G_{n}(t)$ is so. Since orders of $K_{j}(t)$ become lower as $j$ tends to infinity, we conclude that $H_{n}(t)$ belongs to $S^{-\infty}$ if $t$ is positive. $H_{n}(t)$ satisfies a resolvent equation (2.1.8) so that it is the unique solution of (2.1.8).

$$
H_{n}(t)+\int_{0}^{t} H_{n}(s) G_{n}(t-s) d s=H_{n}(t)+\int_{0}^{t} G_{n}(s) H_{n}(t-s) d s=G_{n}(t) .
$$

By the property of the Weyl symbol it is clear that the adjoint operator $(d / d t)-P^{*}$ has also a parametrix $E_{n}(-t)^{*}$ in the negative direction of $t$ and its fundamental solution is given by $E(-t)^{*}$. Since pseudodifferential operators in $L_{0}^{k}$ map $\mathscr{S}$ to $\mathscr{S}$, we may claim the uniqueness of the fundamental solution.

q.e.d.

\section{§ 2.2. An Application. Melin's Result (Gårding Type Inequality)}

In the previous section we proved the existence of the fundamental solution in short $t$. If we define a global one $E(t)$ by products of $E\left(t_{j}\right), t=\sum_{j=1}^{k} t_{j}$; $0 \leqq t_{j} \leqq T, E(t)$ is the fundamental solution of (2.1.2) and has properties of one parameter semigroups as bounded operators on $\boldsymbol{L}^{2}\left(\mathbf{R}^{n}\right)$. We conclude it as the following theorem.

Theorem 2.2. 1) There exists a fundamental solution $E(t)$ of (2.1.2) globally in $t$ which has properties of one parameter semigroups on $\boldsymbol{H}^{\alpha}\left(\mathbf{R}^{n}\right)$ and the estimate (2.2.1), where $c$ is independent of $\alpha$.

$$
\|E(t)\|_{\alpha} \leqq \exp (c t) .
$$

2) $E(t)$ and $(d / d t)^{j} E(t)$ are strongly continuous functions in $t$ valued in pseudodifferential operators $L_{0}^{0}$ and $L_{0}^{m j}\left(S^{-\infty}\right.$ if $\left.t>0\right)$.

3) If we also denote the generator of the semigroup $E(t)$ on $\boldsymbol{H}^{\alpha}\left(\mathbf{R}^{n}\right)$ by 
$-P$ and its definition domain by $D(P)$, there exist constants $\varepsilon>0$ and $\lambda$, which are independent of $\alpha$, such that (2.1.10) holds on $D(P)$, which includes $\mathscr{S}$.

$$
\operatorname{Re}((P+\lambda) u, u)_{\alpha} \geqq \varepsilon\left\|\langle D\rangle^{(m-1) / 2} u\right\|_{\alpha}^{2}, \quad \text { for any } u \text { of } D(P) .
$$

Remark. $(,)_{\alpha}$ and \|\|$_{\alpha}$ mean an inner product and the norm defined by it of Sobolev spaces $\boldsymbol{H}^{\alpha}\left(\mathbf{R}^{n}\right)$. They are not free, namely, depend on $P$.

Proof. It follows from the uniqueness of solutions for $E(t)$ to be well defined. The statement 2) is a direct result of Theorem 1.1. The estimate (2.2.1) is essentially due to $\mathbb{L}^{2}$-boundness of pseudodifferential operators of $L_{0}^{0}$. (Refer to A. P. Calderon and R. Vaillancourt [3].) By them we get the estimate (2.2.3) if the norms are defined by uniformly elliptic pseudodifferential operators.

$$
\|E(t)\|_{\alpha} \leqq M_{\alpha} \exp \left(c_{\alpha} t\right) .
$$

By it we get (2.2.4) integrating $\exp (-\lambda t) E(t)$ in $t$.

$$
\left\|(P+\lambda)^{-1}\right\|_{\alpha} \leqq M_{\alpha}\left(\lambda-c_{\alpha}\right)^{-1} \text { if } \lambda>c_{\alpha} .
$$

We show the statement 3 ) before proving (2.2.1) because it is trivial from the statement 3). Moreover we may restrict it in the case that $\alpha=0$ and that $p$ is $p_{m}+p_{m-1}$ and real, because $\langle\xi\rangle^{-\beta} \circ p_{\circ}\langle\xi\rangle^{\beta}$ has the same principal symbol and the same real part of the subprincipal symbol, and also because real parts of $(Q u, u)_{\alpha}$ for remainder terms $Q$ may seem to be lower than $((P+\lambda) u, u)_{\alpha}$. In this case $P$ with the domain $\mathscr{S}$ is formally selfadjoint on $\mathbb{L}^{2}\left(\mathbb{R}^{n}\right)$. (2.2.4) implies that such $P$ has a selfadjoint extension which is equal to $P$ with the domain $D(P)$, because $(P+\lambda)$ is hypoelliptic by existence of a parametrix $\int_{0}^{T} \exp (-\lambda t) E(t) d t$. Therefore $P$ is a selfadjoint operator bounded below on $\mathbb{E}^{2}\left(\mathbb{R}^{n}\right)$ so that we get (2.2.5) for a constant $\lambda$.

$$
\operatorname{Re}((P+\lambda) u, u)_{0} \geqq 0 .
$$

Condition (A) is satisfied even if $p_{m-1}$ is replaced by $p_{m-1}-\varepsilon\langle\xi\rangle^{m-1}$ for a small $\varepsilon$. So we conclude (2.2.2) in the restricted case. In general we get it adding a sufficiently large $\mathbb{L}^{2}\left(\mathbf{R}^{n}\right)$-norm if $\alpha>0$ and considering the adjoint operator $P^{*}$ if $\alpha<0$.

q.e.d.

\section{Chapter 3. Ambiguities of Complex Phase Functions}

The complex phase function has somewhat its freedom of construction 
as seen in Chapter 1. It may be changed by another one. We shall here give a sufficient condition for the degree of freedom and use it to rewrite the complex phase function in a restricted case.

\section{\$3.1. Replacement of Complex Phase Functions}

It was important for the complex phase function $\phi$ used in Chapter 1 to have satisfied Proposition 1.17. We should consider another one, the function $\phi_{1}$ replaced by which also satisfied it. The following one satisfies it and also the other important properties for replacement.

Let us consider four symbols $q_{j}(j=0,1,2)$ of $L_{1 / 2}^{m-j / 2}$ and $q_{3}$ of $L_{1 / 2}^{m-1}$ satisfying (3.1.1-4), where $1 / 2 \geqq \theta>0$.

$$
\begin{aligned}
& \left|\nabla^{j}\left(p_{m}-q_{0}\right)\right| \leqq c\left(p_{m}^{(2-j) / 2+\theta}+1\right)\langle\xi\rangle^{(m-1) j / 2-m \theta}, \quad(j=0,1,2) \\
& \left|\nabla^{j}\left(\nabla p_{m}-q_{1}\right)\right| \leqq c\left(p_{m}^{(1-j) / 2+\theta}+1\right)\langle\xi\rangle^{(m-1)(j+1) / 2-m \theta}, \quad(j=0,1) \\
& \left|\nabla^{2} p_{m}-q_{2}\right| \leqq c\left(p_{m}^{\theta}+1\right)\langle\xi\rangle^{m-1-m \theta} \\
& \left|p_{m-1}-q_{3}\right| \leqq c\left(p_{m}^{\theta}+1\right)\langle\xi\rangle^{m-1-m \theta}
\end{aligned}
$$

If we formally replace $p_{m}, \nabla p_{m}, \nabla^{2} p_{m}$ and $p_{m-1}$ by $q_{0}, q_{1}, q_{2}$ and $q_{3}$ in (0.2.3), we get (3.1.5).

(3.1.5) $\phi_{1}^{\prime}=-q_{0} t-q_{3} t-\sigma^{1}\left(b^{\prime} t / 2, F\left(A^{\prime} t / 2\right) b^{\prime} t / 2\right)-2^{-1} \operatorname{Tr}\left(\log \left[\cosh \left(A^{\prime} t / 2\right)\right]\right)$, where $b^{\prime}=i J_{1} q_{1}$ and $A^{\prime}=i J_{1} q_{2}$.

We estimate the difference of $\phi_{1}$ and $\phi_{1}^{\prime}$.

Proposition 3.1. Let $g$ be a holomorphic function used in Proposition 1.6. If $X$ is on $\operatorname{supp} \psi_{2}$ and if $(1-2 \varepsilon) \theta-3 \delta>0$, then there exists a positive $T$ such that $g\left(A^{\prime} t / 2\right)$ given in (1.2.17) is well defined on $0 \leqq t \leqq T$ and has the estimate (3.1.6-7) for integers $l \geqq 0$, where $A^{\prime}=i J_{1} q_{2}$.

$$
\begin{aligned}
\| \nabla^{l}(g(A t / 2) & \left.-g\left(A^{\prime} t / 2\right)\right) \| \\
& \leqq c t\langle\xi\rangle^{m-1}\left(1+t\langle\xi\rangle^{m-1}\right)^{2+k}\langle\xi\rangle^{-\varepsilon l+(2 \varepsilon-1) \theta} . \\
\left\|\nabla^{l} g\left(A^{\prime} t / 2\right)\right\| & \leqq c_{l}\left(1+t\langle\xi\rangle^{m-1}\right)^{2 l+k+1}\langle\xi\rangle^{-l / 2} \\
& \leqq c_{l}^{\prime}\left(1+t\langle\xi\rangle^{m-1}\right)^{k+3}\langle\xi\rangle^{-\varepsilon l+(2 \varepsilon-1) \theta}, \text { if } \quad l \geqq 1 .
\end{aligned}
$$

Lemma 3.2. If $X$ is on $\operatorname{supp} \psi_{2}$ and if $(1-2 \varepsilon) \theta-3 \delta>0$, then there exists a positive $T$ for any positive $\varepsilon$ such that (3.1.8) and therefore (3.1.9) hold on 
$0 \leqq t \leqq T$, where $S=A t / 2$ and $S^{\prime}=A^{\prime} t / 2$.

$$
\left\|(\lambda-S)^{-1}\left(S-S^{\prime}\right)\right\| \leqq \varepsilon, \quad \text { if } \quad|\operatorname{Im} \lambda| \geqq 1 .
$$

$$
\left\|\left(\lambda-S^{\prime}\right)^{-1}\right\| \leqq c\left(|\operatorname{Im} \lambda|+t\langle\xi\rangle^{m-1}\right)|\operatorname{Im} \lambda|^{-2}, \quad \text { if } \quad|\operatorname{Im} \lambda| \geqq 1 .
$$

Proof. By Lemma 1.7 the resolvent of $S$ satisfies (1.2.33), that is, (3.1.9) replaced $S^{\prime}$ by $S$ if $t$ is small because $\delta<(1-2 \varepsilon) / 6$. By (3.1.3) $S-S^{\prime}$ satisfies (3.1.10) because $\left|p_{m}\right| \leqq c\langle\xi\rangle^{m-1+2 \varepsilon}$ and $t\langle\xi\rangle^{m-1} \leqq c\langle\xi\rangle^{\delta}$ on supp $\psi_{2}$.

$$
\left\|S-S^{\prime}\right\| \leqq c t\langle\zeta\rangle^{m-1+(2 \varepsilon-1) \theta} \leqq c\langle\xi\rangle^{\delta+(2 \tau-1) \theta} .
$$

So we get (3.1.11) because $3 \delta+(2 \varepsilon-1) \theta<0$.

$$
\left\|(\lambda-S)^{-1}\left(S-S^{\prime}\right)\right\| \leqq c\langle\xi\rangle^{2 \delta+(2 \varepsilon-1) \theta} \leqq c\langle\xi\rangle^{-\delta} .
$$

Therefore (3.1.8) is satisfied if $\langle\xi\rangle$ is sufficiently large. For fixed $\langle\xi\rangle$ we also get it if $t$ is sufficiently small. (3.1.8) implies that the resolvent equation is solvable there and that (3.1.9) holds.

q.e.d.

Proof of Proposition 3.1. It is clear by Lemma 3.2 that $g\left(A^{\prime} t / 2\right)$ is well defined by (1.2.17) and that (3.1.7) holds. (Refer to the proof of Proposition 1.6.) For (3.1.6) we estimate (3.1.12) in the same way as in the proof of Proposition 1.6.

$$
g(S)-g\left(S^{\prime}\right)=(2 \pi i)^{-1} \int_{\Gamma} g(\lambda)(\lambda-S)^{-1}\left(S-S^{\prime}\right)\left(\lambda-S^{\prime}\right)^{-1} d \lambda .
$$

Using (1.2.33), (3.1.9) and $\left\|\nabla^{j}\left(S-S^{\prime}\right)\right\| \leqq c t\langle\zeta\rangle^{m-1}\langle\zeta\rangle^{-\varepsilon j+(2 \varepsilon-1) \theta}$, we get (3.1.6). q.e.d.

Proposition 3.3. The difference of $\phi_{1}$ and $\phi_{1}^{\prime}$ is estimated as (3.1.13-14) on $\left\{\operatorname{supp} \psi_{2}\right\} \times[0, T]$ if $\gamma=(1-2 \varepsilon) \theta-4 \delta-2 \varepsilon>0$.

$$
\begin{aligned}
& \left|\phi_{1}-\phi_{1}^{\prime}\right| \leqq c t\langle\xi\rangle^{m-1-\gamma} . \\
& \left|\nabla^{j}\left(\phi_{1}-\phi_{1}^{\prime}\right)\right| \leqq c t\langle\xi\rangle^{m-1}\left(1+t\langle\xi\rangle^{m-1}\right)^{4}\langle\xi\rangle^{(2-j) \varepsilon+(2 \varepsilon-1) \theta}, \\
& \quad(j=0,1,2, \ldots) .
\end{aligned}
$$

Proof. We estimate the difference of each term using (3.1.6-7) and that $\left|p_{m}\right| \leqq c\langle\xi\rangle^{m-1+2 \varepsilon}$.

$$
\begin{array}{lll}
\text { (3.1.15) } & \left|\nabla^{j}\left(p_{m} t-q_{0} t\right)\right| \leqq c t\langle\xi\rangle^{m-1}\langle\xi\rangle^{(2-j) \varepsilon+(2 \varepsilon-1) \theta}, & (j=0,1,2, \ldots) . \\
\text { (3.1.16) } & \left|\nabla^{j}\left(p_{m-1} t-q_{3} t\right)\right| \leqq c t\langle\xi\rangle^{m-1}\langle\xi\rangle^{-j \varepsilon+(2 \varepsilon-1) \theta}, & (j=0,1,2, \ldots) . \\
\text { (3.1.17) } & \left|\nabla^{j}\left(b t-b^{\prime} t\right)\right| \leqq c t\langle\xi\rangle^{m-1}\langle\xi\rangle^{(1-j) \varepsilon+(2 \varepsilon-1) \theta}, & (j=0,1,2, \ldots) .
\end{array}
$$


We get (3.1.18-19) because the functions $F(A t / 2), F\left(A^{\prime} t / 2\right), \log [\cosh (A t / 2)]$ and $\log \left[\cosh \left(A^{\prime} t / 2\right)\right]$ satisfy $(3.1 .6-7)$ with $k=0$.

$$
\begin{aligned}
& \left|\nabla^{j}\left\{\sigma^{1}(b t / 2, F(A t / 2) b t / 2)-\sigma^{1}\left(b^{\prime} t / 2, F\left(A^{\prime} t / 2\right) b^{\prime} t / 2\right)\right\}\right| \\
& \quad \leqq c\left(t\langle\xi\rangle^{m-1}\right)^{2}\left(1+t\langle\xi\rangle^{m-1}\right)^{3}\langle\xi\rangle^{(2-j) \varepsilon+(2 \varepsilon-1) \theta}, \quad(j=0,1,2, \ldots) . \\
& \mid \nabla^{j}\left\{\operatorname{Tr}(\log [\cosh (A t / 2)])-\operatorname{Tr}\left(\log \left[\cosh \left(A^{\prime} t / 2\right]\right)\right\} \mid\right. \\
& \quad \leqq c\left(t\langle\xi\rangle^{m-1}\right)\left(1+t\langle\xi\rangle^{m-1}\right)^{2}\langle\xi\rangle^{-\varepsilon j+(2 \varepsilon-1) \theta}, \quad(j=0,1,2, \ldots) .
\end{aligned}
$$

Summing up these and noting $\gamma=(1-2 \varepsilon) \theta-4 \delta-2 \varepsilon>0$ we get (3.1.13-14).

q.e.d.

Let us define $\phi^{\prime}$ by (3.1.20). (Refer to (0.2.6).)

$$
\phi^{\prime}=\psi_{1} \phi_{1}^{\prime}+\left(1-\psi_{1}\right) \phi_{2} .
$$

Using the parametrix with respect to $\phi$ (Theorem 1.1) we construct a parametrix with respect to $\phi^{\prime}$, that is, we approximate $\exp \phi$ by products of $\exp \phi^{\prime}$ and some amplitude functions. Proposition 3.3 guarantees for $\phi^{\prime}$ to be able to be another complex phase function and for the quotient $\exp \left(\phi-\phi^{\prime}\right)$ of $\exp \phi$ by $\exp \phi^{\prime}$ to be replaced by the Taylor's series as powers of $\left(\phi-\phi^{\prime}\right)$. Let us write it as the next proposition.

Let us put the Taylor's series of $\exp \lambda$ and its remainder terms as (3.1.21).

$$
\begin{aligned}
& r_{n}(\lambda)=\sum_{j=0}^{n}(j !)^{-1} \lambda^{j}, \\
& r_{n}^{\prime}(\lambda)=\exp \lambda-r_{n}(\lambda) .
\end{aligned}
$$

Proposition 3.4. Let us assume that $\gamma=(1-2 \varepsilon) \theta-4 \delta-2 \varepsilon>0$ and that $(1-2 \varepsilon) \theta-3 \varepsilon \geqq 0$.

1) $\exp \phi^{\prime}$ has the same estimate (3.1.22) as $\exp \phi$, where $\phi_{0}=\Phi_{2} \psi_{1}+$ $t p_{m}\left(1-\psi_{1}^{1}\right)+c t\langle\xi\rangle^{m-1},(c>0)$. (Refer to Proposition 1.25.)

$$
\left|\exp \phi^{\prime}\right| \leqq c \exp \left(-\phi_{0}\right) \text {. }
$$

2) $\phi-\phi^{\prime}$ belongs to $N_{g}(1,0,-\varepsilon, 0)+N_{g}^{-\infty}$. Therefore $\nabla \phi^{\prime}$ belongs to $N_{g}^{*}(1,1,0,0)+N_{g}^{-\infty}$. (Refer to Section 1.3 and Proposition 1.27.)

3) There exist $g_{j}$ and $g_{j}^{\prime}$ of $N_{g}^{-\infty}$ such that (3.1.23-24) hold, where $c_{0}$ is independent of $n$ and $j$.

$$
\begin{aligned}
& \left|\nabla^{j} \exp \pm\left(\phi-\phi^{\prime}\right)\right| \\
& \quad \leqq\left(c_{n j}\left(1+t\langle\xi\rangle^{m-1}\right)^{d(n, j)}\langle\xi\rangle^{-j \varepsilon}+g_{j}\right) \exp \left(c_{0} t\langle\xi\rangle^{m-1-\gamma}\right) .
\end{aligned}
$$

(3.1.24) $\left|\nabla^{j} r_{n}^{\prime}\left(\phi-\phi^{\prime}\right)\right|$

$$
\begin{aligned}
\leqq\left(c_{n j}\left(t\langle\xi\rangle^{m-1}\right)^{n+1}\left(1+t\langle\xi\rangle^{m-1}\right)^{d(n, j)}\right. & \langle\xi\rangle^{-(n+1+j) \varepsilon} \\
& \left.+g_{j}^{\prime}\right) \exp \left(c_{0} t\langle\xi\rangle^{m-1-\gamma}\right) .
\end{aligned}
$$


4) $\exp \phi^{\prime}$ belongs to $L_{0}^{0}$.

5) Let $f_{j}$ be the amplitude functions of the parametrix $E_{n}(t)$ with respect to $\phi$ Theorem 1.1. Then $f_{j} r_{n}^{\prime}\left(\phi-\phi^{\prime}\right) \exp \phi^{\prime}$ belongs to $L_{0}^{-(n+1+j) \varepsilon}$.

6) Let us put $((d / d t)+p)_{{ }_{(2)}} \exp \phi^{\prime}=g_{0} \exp \phi^{\prime}$. Then $g_{0}$ belongs to $N_{g}^{* *}(1,1, m-1-\varepsilon, 0)+N_{g}^{* *}(0,0, m-1,1)+N_{g}^{-\infty}$. (Refer to Proposition 1.30.)

Proof. At first we note that $\phi-\phi^{\prime}=\psi_{1}\left(\phi_{1}-\phi_{1}^{\prime}\right)$. So we get (3.1.25) by (3.1.13), and (3.1.26) by combining (3.1.14) with (1.3.43).

$$
\begin{aligned}
& \left|\phi-\phi^{\prime}\right| \leqq c t\langle\xi\rangle^{m-1-\gamma} . \\
& \phi-\phi^{\prime} \text { belongs to } N_{g}(1,0,2 \varepsilon+(2 \varepsilon-1) \theta, 0)+N_{g}^{-\infty} .
\end{aligned}
$$

1) By Proposition 1.25, (3.1.22) holds for $\phi$ so that $\left|\exp \phi^{\prime}\right| \leqq|\exp \phi|$ $\times\left|\exp \left(\phi-\phi^{\prime}\right)\right| \leqq c \exp \left(-\phi_{0}\right) \exp c t\langle\xi\rangle^{m-1-\gamma}$. Since $\phi_{0}$ is bounded below by $c t\langle\xi\rangle^{m-1}$ with a positive constant, (3.1.22) holds for $\phi^{\prime}$.

2) Since we have assumed that $2 \varepsilon+(2 \varepsilon-1) \theta<-\varepsilon$, (3.1.26) implies the statement 2). For $\nabla \phi^{\prime}$ it follows from the result for $\phi$.

3) Since $\nabla^{j} \exp \pm\left(\phi-\phi^{\prime}\right)$ and $\nabla^{j} r_{n}^{\prime}\left(\phi-\phi^{\prime}\right)$ are bounded by the products of $\exp \left|\phi-\phi^{\prime}\right|$ and derivatives of $\left(\phi-\phi^{\prime}\right)$, we get (3.1.23-24) by combining with 2). (Refer to (1.3.36).)

4) and 5) We consider that $\exp \phi^{\prime}=\exp \phi \exp \left(\phi^{\prime}-\phi\right)$ and $f_{j} r_{n}^{\prime}\left(\phi-\phi^{\prime}\right)$ $\times \exp \phi^{\prime}=f_{j} \exp \phi r_{n}^{\prime}\left(\phi-\phi^{\prime}\right) \exp \left(\phi^{\prime}-\phi\right) . \quad \nabla^{l} \exp \phi$ and $\nabla^{l} f_{j} \exp \phi$ are bounded by $\exp \left(-\phi_{0} / 2\right)$ and $\langle\xi\rangle^{-\varepsilon j} \exp \left(-\phi_{0} / 2\right)$, respectively, according to Proposition 1.28. Combining them with (3.1.23-24) we conclude the statements 4) and 5).

6) At (1.6.53) we replace $\phi$ by $\phi^{\prime}$ using 2) and (3.1.1-4) if it is necessary. Then we get the statement 6$)$.

q.e.d.

Theorem 3.1. Let us consider four pseudodifferential operators satisfying (3.1.1-4) and define $\phi^{\prime}$ by (3.1.20). We add it to the conditions at Theorem 1.1 that $(1-2 \varepsilon) \theta-4 \delta-2 \varepsilon>0$ and $(1-2 \varepsilon) \theta-3 \varepsilon \geqq 0$. There exist $f_{j}^{\prime}$ belonging to $M(j)(j=1,2, \ldots)$, therefore $f_{j}^{\prime} \exp \phi^{\prime}$ belongs to $L_{0}^{-\varepsilon j}$, such that $((d / d t)+p)^{\circ}{ }_{(n+2)}$ $\left(\sum_{j=0}^{n} f_{j}^{\prime}\right) \exp \phi^{\prime}=G_{n}$ belongs to $L_{0}^{m-1-(n+1) \varepsilon}$ if $n \geqq 1$.

Proof. Let us define $f_{j}^{\prime}$ by $f_{j}^{\prime}=\sum_{j=k+l} f_{k}(l !)^{-1}\left(\phi-\phi^{\prime}\right)^{l}$. Then it is clear that $f_{j}^{\prime}$ belongs to $M(j)$. (Refer to Theorem 1.1 and 2) of Proposition 3.4.) We consider $\left(\sum_{j=0}^{n} f_{j}\right) r_{n}\left(\phi-\phi^{\prime}\right) \exp \phi^{\prime}$, which is equal to $\left(\sum_{j=0}^{n} f_{j}\right) \exp \phi$ $-\left(\sum_{j=0}^{n} f_{j}\right) r_{n}^{\prime}\left(\phi-\phi^{\prime}\right) \exp \phi^{\prime}$. (Refer to (3.1.21).) $((d / d t)+p)_{(n+2)}\left(\sum_{j=0}^{n} f_{j}\right) \exp \phi$ belongs to $L_{0}^{m-1-\varepsilon(n+1)}$ by Theorem 1.1 and $((d / d t)+p){ }_{(n+2)}\left(\sum_{j=0}^{n} f_{j}\right) r_{n}^{\prime}\left(\phi-\phi^{\prime}\right)$ $\times \exp \phi^{\prime}$ belongs to $L_{0}^{m-(n+1+j) \varepsilon}$ by 5) of Proposition 3.4. So we get that 
$((d / d t)+p)^{\circ}{ }_{(n+2)}\left(\sum_{j=0}^{n} f_{j}\right) r_{n}\left(\phi-\phi^{\prime}\right) \exp \phi^{\prime}$ belongs to $L_{0}^{m-(n+1) \varepsilon} . f_{k}\left(\phi-\phi^{\prime}\right)^{l}$ $\times \exp \phi^{\prime}$ also belongs to $L_{0}^{-\varepsilon j}$ if $j=k+l$ so that $((d / d t)+p)^{\circ}{ }_{(n+1)} f_{k}\left(\phi-\phi^{\prime}\right)^{l} \exp \phi^{\prime}$ belongs to $L_{0}^{m-(n+1) \varepsilon}$ if $\left(k+l^{\prime}\right) \geqq n+1$. Therefore we conclude that $((d / d t)+p)$ ${ }^{\circ}(n+1)\left(\sum_{j=0}^{n} f_{j}^{\prime}\right) \exp \phi^{\prime}$ belongs to $L_{0}^{m-(n+1) \varepsilon}$.

Now we replace $n$ by $n+k(k \varepsilon \geqq 1)$.

$$
\begin{aligned}
&((d / d t)+p)^{\circ}{ }_{(n+1)}\left(\sum_{j=0}^{n} f_{j}^{\prime}\right) \exp \phi^{\prime} \\
&=((d / d t)+p)_{(n+k+1)}\left(\sum_{j=0}^{n+k} f_{j}^{\prime}\right) \exp \phi^{\prime} \\
&-\sum_{l=n+2}^{n+k+1} \sigma_{l}\left(p,\left(\sum_{j=0}^{n+k} f_{j}^{\prime}\right) \exp \phi^{\prime}\right) \\
&-((d / d t)+p)_{(n+1)}\left(\sum_{j=n+1}^{n+k} f_{j}^{\prime}\right) \exp \phi^{\prime} .
\end{aligned}
$$

Noting (3.1.27) we get the conclusion. In fact it is clear that $\sigma_{l}\left(p, f_{j}^{\prime} \exp \phi^{\prime}\right)$ belongs to $L_{0}^{m-1-(n+1) \varepsilon}$ if $l \geqq 3$ and $j \geqq n+1$ or if $l \geqq n+2$ and $j \geqq 0$. Therefore we have only to show that $((d / d t)+p){ }^{\circ}{ }_{(2)} f_{j}^{\prime} \exp \phi^{\prime}$ belongs to $L_{0}^{m-1-(n+1) \varepsilon}$ if $j \geqq n+1$. It is equal to $f_{j}^{\prime}((d / d t)+p)^{\circ}{ }_{(2)} \exp \phi^{\prime}+(2 i)^{-1} \sigma\left(\nabla p, \nabla f_{j}^{\prime}\right) \exp \phi^{\prime}+$ $(2 i)^{-2} 2^{-1} \sigma\left(\nabla^{2} p, \nabla^{2} f_{j}^{\prime}+\nabla f_{j} \nabla \phi^{\prime}\right) \exp \phi^{\prime}$, which belongs to $L_{0}^{m-1-(n+1) \varepsilon}$ by 2$)$ and 6) of Proposition 3.4.

q.e.d.

\section{§3.2. Special Case (Exact Double Characteristic)}

We apply Theorem 3.1 to the case that the principal symbol $p_{m}$ has only exact double characteristics, namely, $p_{m}(X) \geqq c(X) d(X, \Sigma)^{2},(X=(x, \xi), \xi \neq 0)$ for a positive continuous function $c(X)$ where $d(X, \Sigma)$ is the distance of $X$ to the characteristic set $\Sigma$ in $\mathbb{R}^{n} \times \mathbf{R} \times \mathrm{S}^{n-1}$. So we assume it through this section. In this case we get a similar form of complex phase functions to the case that an operator $P$ is given by a quadratic form in $(x, \xi)$. (Refer to examples in the introduction.)

We consider an infinilely differentiable mapping $a$, satisfying (3.2.1-2) of a conic neighborhood of the characteristic set $\Sigma$ to $\Sigma$.

$$
|d(X, a(X))-d(X, \Sigma)| \leqq c d(X, \Sigma)^{2} .
$$

We put $(y, \eta)=a(X), X=(x, \xi)$.

$$
\left(|\eta|^{1 / 2} y,|\eta|^{-1 / 2} \eta\right) \text { has the homogeneous order of } 1 / 2 \text { in } \xi \text {. }
$$

In fact $d(X, \Sigma)$ is an infinitely differentiable function on a conic neighborhood of $\Sigma$ because $\Sigma$ is an infinitely differentiable submanifold of $\mathbf{R}^{n} \times \mathbf{R}^{n} \backslash\{0\}$. A mapping $a(X)$ attaining $d(X, a(X))=d(X, \Sigma)$ is infinitely differentiable on a conic neighborhood of $\Sigma$ and satisfies (3.2.1-2) there. 
Let us define $q_{0}, q_{1}, q_{2}$ and $q_{3}$ by (3.2.3), where $H_{p}(X)$ is the Hesse matrix of $p_{m}(X)$ in $X$, where $a=a(X)$.

$$
\begin{aligned}
& q_{0}=2^{-1}\left\langle X-a, H_{p}(a)(X-a)\right\rangle, \\
& q_{1}=H_{p}(a)(X-a), \\
& q_{2}=H_{p}(a)
\end{aligned}
$$

and

$$
q_{3}=p_{m-1}(a) \text {. }
$$

Proposition 3.5. $q_{j}(j=0,1,2,3)$ defined by (3.2.3) satisfy (3.1.1-4) with $\theta=1 / 2$, where constants $c$ are uniformly bounded on compact sets of $x$-space.

Proof. Let us consider the Taylor's expansion of a symbol $f(X)$ of $L_{1 / 2}^{m}$ at $Y$, where $Y=(y, \eta), Z=(z, \zeta)=X-Y$ and $Z^{\sim}=\left(\langle\eta\rangle^{1 / 2} z,\langle\eta\rangle^{-1 / 2} \zeta\right)$.

$$
\begin{aligned}
& f(X)=f(Y)+\langle d f, X-Y\rangle+2^{-1}\left\langle X-Y,\left(d^{2} f\right)(X-Y)\right\rangle+R(X, Y) \\
& \quad=f(Y)+\left\langle\nabla f, Z^{\sim}\right\rangle+2^{-1}\left\langle Z^{\sim}, \nabla^{2} f Z^{\sim}\right\rangle+R(X, Y) . \\
& \left|\nabla_{X}^{k} R(X, Y)\right|+\left|\nabla_{Y}^{k} R(X, Y)\right| \leqq|Z|^{3-k} c(X, Y), \quad(k=0,1,2,3) .
\end{aligned}
$$

Let us put $f=p_{m}$ and $Y=a(X)$. Then we get $p_{m}(X)-q_{0}(X)=R(X, a(X))$ because $p_{m}(a)=\nabla p_{m}(a)=0$. Since $p_{m}$ and $q_{0}$ belong to $L_{1 / 2}^{m}, R(X, a(X))$ also belongs to $L_{1 / 2}^{m}$ so that (3.2.6) is valid according to (3.2.5).

$$
\left|\nabla_{X}^{k} R(X, a(X))\right| \leqq c\left(\left|Z^{\sim}\right|^{3-k}+1\right)\langle\xi\rangle^{m-3 / 2}, \quad(k=0,1,2,3) .
$$

Since $\left|Z^{\sim}\right|^{2} \leqq c p_{m}\langle\xi\rangle^{1-m}+1$ by the assumption, we obtain (3.1.1) with $\theta=1 / 2$. (3.1.2-4) are proved by considering cases that $f$ is $\nabla p_{m}, \nabla^{2} p_{m}$ or $p_{m-1}$ as well as the above.

q.e.d.

Theorem 3.2. Let us assume that the principal symbol $p_{m}$ has only exact double characteristics. Theorem 3.1 is valid with $\theta=1 / 2$ if $\phi_{1}$ is replaced by $\phi_{3}$ (3.2.7), where the asymptotic expansion is uniform on each compact set of $x$-space.

$$
\begin{aligned}
\phi_{3}= & -p_{m-1}(a) t+i \sigma^{1}\left((X-a)^{\sim}, \tanh (A(a) t / 2)(X-a)^{\sim}\right) \\
& -2^{-1} \operatorname{Tr}(\log [\cosh (A(a) t / 2)]),
\end{aligned}
$$

where $a$ is a mapping satisfying (3.2.1), $A(X)=i J_{1} \nabla^{2} p_{m}(X)$ and $(X-a)^{\sim}=$ $\left(\langle\eta\rangle^{1 / 2} z,\langle\eta\rangle^{-1 / 2} \zeta\right)$ for $Y=(y, \eta)=a(X)$ and $Z=(z, \zeta)=X-Y$.

Proof. Since a constructed parametrix would be a pseudodifferential operator of $L_{0}$, it would have pseudolocal property. Therefore we may assume that the conditions (3.1.1-4) are uniformly satisfied by $q_{j}$ of (3.2.3) on the whole 
space. Then we get a parametrix according to Theorem 3.1 if $8 \varepsilon \leqq 1$. Noting (3.2.8) we get the conclusion.

$$
i A t / 2+(A t / 2) F(A t / 2)(A t / 2)=i \tanh (A t / 2) .
$$

q.e.d.

\section{Chapter 4. Asymptotic Behavior of Trace}

We consider a Cauchy problem of parabolic type on a compact infinitely differentiable manifold. A parametrix for it makes it possible to calculate the rate of the trace of the fundamental solution as $t$ tends to zero. Karamata's Tauberian Theorem gives an information about distribution of eigenvalues for a stationary problem.

\section{§4.1. Assumptions and Conclusions}

Let $M$ be an $n$-dimensional infinitely differentiable manifold with a fixed positive smooth density $d M . \quad P$ is a classical pseudodifferential operator of order $m(m>1)$ and formally selfadjoint with respect to the density $d M$.

$$
\int_{M} P u v d M=\int_{M} u \underline{P v} d M \text { for } u \text { and } v \text { of } C^{+\infty}(M)
$$

Let $p$ be a symbol of $P$, that is, $p$ gives a local representation of $P$. (Refer to Appendix.) The principal symbol $p_{m}$ and the subprincipal symbol $p_{m-1}$ of $p$, therefore, of $P$ are well defined on $T^{*} M$. And the condition (4.1.1) implies that $p_{m}$ and $p_{m-1}$ are real valued. So the statement of Condition (A) is well defined on $M$. We assume it to $P$.

Let us consider a Cauchy problem (4.1.2).

$$
\begin{aligned}
& ((d / d t)+P) u=f \\
& \left.u\right|_{t=0}=g .
\end{aligned}
$$

Since parametrices constructed in the previous chapters were pseudodifferential operators of $L_{0}^{0}$, they have pseudolocal properties so that the discussions in Chapter 2 is valid on a compact manifold $M$. We execute it to obtain that the fundamental solution $E(t)$ of (4.1.2) is also a pseudodifferential operator of $L_{0}^{0}$ and a smooth kernel if $t$ is positive and that the parametrix $E_{n}(t)$ of Theorem 1.1 with respect to a fixed local coordinate is a local parametrix of $E(t)$. If $t$ is posi- 
tive, $E(t)$ is an integral operator with a smooth kernel $E(t, x, y)$ as noting above, that is,

$$
(E(t) u)(x)=\int_{M} E(t, x, y) u(y) d M
$$

Therefore $\operatorname{Tr} E(t)$ is given by (4.1.4).

$$
\operatorname{Tr} E(t)=\int_{M} E(t, x, x) d M
$$

Let us fix a local chart $(x, U)$. It is written as (4.1.5) with a symbol $e(t, x, \xi)$ of $E(t)$, where $G(t, x)$ is a smooth function in $(x, t)$.

$$
\int_{U} E(t, x, x) d M=(2 \pi)^{-n} \int_{T^{*} U} e(t, x, \xi) d x d \xi+\int_{U} G(t, x) d M
$$

Moreover if we consider a parametrix $E_{n}(t)$ with a symbol $e_{n}(t, x, \xi)$ for $E(t)$ on $U$, we obtain (4.1.6) with some sufficiently smooth function $G_{n}(t, x)$.

$$
\begin{aligned}
& (2 \pi)^{-n} \int_{T^{*} U} e(t, x, \xi) d x d \xi \\
& \quad=(2 \pi)^{-n} \int_{T^{*} U} e_{n}(t, x, \xi) d x d \xi+\int_{U} G_{n}(t, x) d M .
\end{aligned}
$$

Since $\int G(t, x) d M$ and $\int G_{n}(t, x) d M$ are bounded even if $t$ tends to zero, it suffices to calculate the rate of $\int_{T^{*} U} e_{n}(t, x, \xi) d x d \xi$. Thus we obtain the following theorem.

Theorem 4.1. Let $(x, U)$ be a local chart of $M$ and $\phi$ be a complex phase function defined by (0.2.3-8) with respect to a local coordinate $x$ on $U$. Then we get (4.1.7) as tends to zero.

$$
\int_{U} E(t, x, x) d M=(1+o(1))(2 \pi)^{-n} \int_{T^{*} U} \exp \phi d x d \xi
$$

Remark. $\exp \phi$ is real positive by construction.

Proof. At first we note that $E_{n}(t)$ is written as (4.1.8) with $f$ satisfying (4.1.9) according to Theorem 1.1 and Proposition 1.26.

$$
\begin{aligned}
& E_{n}(t)=(1+f) \exp \phi \\
& |f| \leqq c\left(1+\phi_{0}\right)^{d}\langle\xi\rangle^{-\varepsilon} .
\end{aligned}
$$

We prove that $|f \exp \phi| \leqq \theta \exp \phi+c_{1} \exp \left(-c_{2}\langle\xi\rangle^{\mu}\right)$ for any positive $\theta$, where $c_{1}$ and $c_{2}$ may depend on $\theta$. We assume that $c\left(1+\phi_{0}\right)^{d}\langle\xi\rangle^{-\varepsilon} \geqq 0$. Then $1+\phi_{0}$ 
$\geqq 2 c_{2}\langle\xi\rangle^{\mu}$ where $\mu=\varepsilon / d$. Since $\exp \phi \leqq c \exp \left(-\phi_{0}\right)$ by Proposition 1.25, $|f \exp \phi| \leqq c_{3} \exp \left(-\phi_{0} / 2\right) \leqq c_{1} \exp \left(-c_{2}\langle\xi\rangle^{\mu}\right)$. Therefore we get (4.1.10).

$$
\left|\int_{T * U} f \exp \phi d x d \xi\right| \leqq \theta \int_{T * U} \exp \phi d x d \xi+M_{\theta} \text {. }
$$

By the way $\exp \phi \geqq c \exp \left(-c^{\prime} t\langle\xi\rangle^{m}\right)$ if $t\langle\xi\rangle^{m-1} \leqq \delta$ for a sufficiently small positive $\delta$. This implies that $\int_{T^{*} U} \exp \phi d x d \xi \geqq c t^{-n / m}$. Therefore for any $\theta>0$, we get (4.1.11) as $t$ tends to zero, which means (4.1.7).

$$
\left|\int_{T^{*} U} f \exp \phi d x d \xi\right| \leqq 2 \theta \int_{T^{*} U} \exp \phi d x d \xi \text { q.e.d. }
$$

Remark. Let $\Xi(x, \xi)$ be an elliptic symbol such that $c\langle\xi\rangle \leqq \Xi(x, \xi) \leqq c^{\prime}\langle\xi\rangle$. Let $\phi^{\prime}$ stand for $\phi$ in which $\langle\xi\rangle$ is replaced to $\Xi(x, \xi)$. Then $\int_{T^{*} U} \exp \phi d x d \xi$ in (4.1.7) is able to be replaced to $\int_{T^{*} U} \exp \phi^{\prime} d x d \xi$. (Refer to Section 4.4.) This implies Theorem 0.3 .

Remark. We consider the above proof dividing the domain $T^{*} U$ in three parts such that

$$
\begin{aligned}
& \Omega 1=T^{*} U \wedge\left\{p_{m}(X) \leqq\langle\xi\rangle^{m-1+2 \varepsilon} \text { and } t\langle\xi\rangle^{m-1} \leqq\langle\xi\rangle^{\delta}\right\} \\
& \Omega 2=T^{*} U \wedge\left\{p_{m}(X) \geqq\langle\xi\rangle^{m-1+2 \varepsilon}\right\}
\end{aligned}
$$

and

$$
\Omega 3=T^{*} U \backslash\{\Omega 1 \wedge \Omega 2\},
$$

where $\delta$ and $\varepsilon$ satisfy (0.2.8). Then we can exclude the cut off function $\psi_{j}$ from the integrand, namely, we get (4.1.13).

Corollary of Theorem 4.1. The right hand side of (4.1.7) may be changed as followings.

$$
\begin{aligned}
& \int_{U} E(t, x, x) d M \\
& \quad=(1+o(1))(2 \pi)^{-n}\left\{\int_{\Omega 1} \exp \phi_{1} d x d \xi+\int_{\Omega 2} \exp \left(-t p_{m}\right) d x d \xi\right\}
\end{aligned}
$$

Proof. In (4.1.10) replace the domain $T^{*} U$ by $\Omega 1$ or $\Omega 2$ and consider $\phi$ with respect to $\delta^{\prime}$ and $\varepsilon^{\prime}$ which are larger than $\delta$ and $\varepsilon$ or smaller than $\delta$ and $\varepsilon$. Then $\exp \phi_{1}=\exp \phi$ on $\Omega 1$ and $\exp \left(-t\left(p_{m}+\langle\xi\rangle^{m-1}\right)\right)=\exp \phi$ on $\Omega 2$. q.e.d.

A restriction of the principal symbol $p_{m}$ is necessary for more precise calculations of rate at the right hand side of (4.1.7) or (4.1.13).

We assume Condition (B), that is, the principal symbol $p_{m}$ has the exactly 
double characteristics. Then we get the followings.

In this case the characteristic set $\Sigma=\left\{p_{m}=0\right\}$ is an infinitely differentiable conic submanifold of $T^{*} M$ as noting at Introduction or Chapter 3. Let $\Sigma^{i}$ be submanifolds of $\Sigma$ such that $\operatorname{codim} \Sigma^{i}=d_{i}$. Defining $\operatorname{codim} \Sigma=d$ by $d=\min \left\{d_{i}\right\}$, we put $\Sigma^{0}=\vee_{d=d_{i}} \Sigma^{i}$.

Theorem 4.2. We assume Condition $(A)$ and $(B)$. Trace of the fundamental solution $\operatorname{Tr} E(t)$ has the asymptotic behaviors (4.1.14) as t tends to zero, where $C_{i}$ are given by (4.1.15).

or

$$
=\left(C_{3}+o(1)\right) t^{-(n-d / 2) /(m-1)} \quad \text { if } \quad n-m d / 2>0 \text {. }
$$

$$
\begin{aligned}
C_{1} & =(2 \pi)^{-n} \int_{T^{*} M} \exp \left(-p_{m}\right) d x d \xi \\
C_{2} & =m^{-1}(2 \pi)^{-(n-d / 2)} \int_{D} d \Sigma_{s}^{0}, D=\Sigma_{s}^{0} \\
& =m^{-1}(2 \pi)^{-(n-d / 2)} \int_{\Sigma^{0}}\left(p_{m-1}+2^{-1} \operatorname{Tr}^{\sim} A\right) \exp \left(-p_{m-1}-2^{-1} \operatorname{Tr}^{\sim} A\right) d \Sigma^{0}
\end{aligned}
$$

and

$$
C_{3}=(2 \pi)^{-(n-d / 2)} \int_{2^{0}}\left[\operatorname{det}\left\{(A / 2)^{-1} \sinh (A / 2)\right\}\right]^{-1 / 2} \exp \left(-p_{m-1}\right) d \Sigma^{0}
$$

Remark. $d \Sigma^{0}$ is an induced density on $\Sigma^{0}$ by $p_{m}$ and $d x d \zeta$. $d \Sigma_{s}^{0}$ is its induced density on $\Sigma_{s}^{0}$ the image of $\Sigma^{0}$ into the spherical bundle $S^{*} M$. (Refer to Introduction or Section 4.3.)

We consider another restriction that $\int_{T^{*} M} \exp \left(-p_{m}\right) d x d \xi$ is finite, that is, $\int_{\left\{p_{m} \leqq 1\right\}} d x d \xi$ is finite. In this case we get the same result as the first case of

Theorem 4.3. We assume Condition $(A)$ and that $\int_{T^{*} M} \exp \left(-p_{m}\right) d x d \xi$ is finite. Then $\operatorname{Tr} E(t)$ has the asymptotic behavior (4.1.16) as $t$ tends to zero.

$$
\operatorname{Tr} E(t)=\left\{C_{1}+o(1)\right\} t^{-n / m},
$$

where $C_{1}=(2 \pi)^{-n} \int_{T^{*} M} \exp \left(-p_{m}\right) d x d \xi$. 


\section{§4.2. Preparations}

We start from the following proposition to calculate the rate. Using Theorem 3.2 of Chapter 3, it is obtained as well as Theorem 4.1 though $\varepsilon$ should be restricted as $8 \varepsilon<1$.

Proposition 4.1. Let $(x, U)$ be a local chart of $M$.

$$
\begin{aligned}
& \int_{U} E(t, x, x) d M \\
& =(1+o(1))(2 \pi)^{-n}\left(\int_{\Omega 1} \exp \phi_{3} d x d \xi+\int_{\Omega 2} \exp \left(-t p_{m}\right) d x d \xi\right)
\end{aligned}
$$

as $t$ tends to zero, where $\Omega 1$ and $\Omega 2$ are ones used in the previous section.

Therefore we shall calculate only $\int_{\Omega 1} \exp \phi_{3} d x d \xi$ and $\int_{\Omega 2} \exp \left(-t p_{m}\right) d x d \xi$. To do so we further subdivide the domain $T^{*} U$, that is, we consider the integrals on a conic neighborhood of a point $X$ in $T^{*} U$ and gather them after obtaining the rate. For simplicity of calculation, we identify $U$ to an open set of $\mathbf{R}^{n}$. Let us consider a conic neighborhood $\Omega$ of $X$ and denote $\Omega 1 \wedge \Omega$ and $\Omega 2 \wedge \Omega$ by the same notations $\Omega 1$ and $\Omega 2$. In case that the closure of $\Omega$ does not intersect the characteristic set $\Sigma$, it is easy. In fact $\int_{\Omega 1} \exp \phi_{3} d x d \xi$ is uniformly bounded in $t$ and $\int_{\Omega 2} \exp \left(-t p_{m}\right) d x d \xi=(1+o(1)) t^{-n / m} \int_{\Omega} \exp \left(-p_{m}\right) d x d \xi$. Thus it suffices to calculate them in case that $\Omega$ includes a point $X$ of the characteristic set $\Sigma$. So we assume that $\Omega$ satisfies the following properties, where we denote the intersection of $\Omega$ and $\Sigma$ also by $\Sigma$ and assume that $\operatorname{codim} \Sigma=d$. (It may be different from $\operatorname{codim} \Sigma$ in the total domain.)

(4.2.2) There exist an open set $U$ of $\mathbf{R}^{2 n-1-d}$ and an infinitely differentiable mapping $\tau(\omega, r, y)$ from $U \times \mathbf{R}_{+} \times \mathbf{R}^{d}$ to $T^{*} \mathbf{R}^{n}$ such that $\tau$ is a local diffeomorphism from $U \times \mathbf{R}_{+} \times\{|y|<L\}$ onto $\Omega$ and satisfies (4.2.3-6).

$$
\tau(\omega, r, y)=\tau_{0}(\omega, r)+\tau_{1}(\omega, r) y=(x, \breve{\zeta}) .
$$

(4.2.4) $\tau_{0}(\omega, r)$ is a diffeomorphism from $U \times \mathbf{R}_{+}$onto $\Sigma$, especially $\tau_{0}(\omega, r)$ is a diffeomorphism from $U$ onto the intersection $\Sigma_{1}$ of $\Sigma$ and $S^{*} \mathbf{R}^{n}$.

(4.2.5) If we define a mapping $a(X)$ from $\Omega$ to $\Sigma$ by $a(X)=\tau_{0}(\omega, r)=$ $a(\tau(\omega, r, y))$ for $X=\tau(\omega, r, y), a$ is an infinitely differentiable mapping satisfying (3.2.1-2). 
(4.2.6)

$\tau^{(1)}(\omega, s r, y)=\tau^{(1)}(\omega, r, y)$ and $\tau^{(2)}(\omega, s r, y)=s \tau^{(2)}(\omega, r, y)$, where $x=\tau^{(1)}(\omega, r, y)$ and $\xi=\tau^{(2)}(\omega, r, y)$ when $(x, \breve{\zeta})=\tau(\omega, r, y)$. In this case we immediately get (4.2.10) through (4.2.7-9).

(4.2.8) $\left.(\partial \tau / \partial(\omega, r, y))\right|_{y=0}$

$$
=\left.\left[\operatorname{det}\left\{{ }^{t}\left(\partial \tau_{0} / \partial(\omega, r)\right)\left(\partial \tau_{0} / \partial\left(\omega, r^{r}\right)\right)\right\} \operatorname{det}\left\{{ }^{t} \tau_{1} \tau_{1}\right\}\right]^{1 / 2}\right|_{r=1} r^{n-1} \text {. }
$$

Remark. $T_{x}(\Sigma), x=\tau_{0}(\omega, r)$, is orthogonal to range $\tau_{1}(\omega, r)$.

$$
\begin{aligned}
& \left.\operatorname{det}\left\{{ }^{\prime}\left(\partial \tau_{0} / \partial(\omega, r)\right)\left(\partial \tau_{0} / \partial(\omega, r)\right)\right\}\right|_{r=1} \\
& \quad=\left.\left.\operatorname{det}\left\{{ }^{r}(\partial \tau / \partial \omega)(\partial \tau / \partial \omega)\right\}\right|_{r=1}\left|\tau_{0}^{(2)}\right|^{2}\right|_{r=1} .
\end{aligned}
$$

Remark. $\Sigma_{1}$, the intersection of $\Sigma$ and $S^{*} \mathbb{R}^{n}$, is orthogonal to the radial direction.

$$
\begin{aligned}
& \left.(\partial \tau / \partial(\omega, r, y))\right|_{y=0} d \omega d r \\
& \quad=\left.\left[\operatorname{det}\left\{t\left(\partial \tau_{0} / \partial \omega\right)\left(\partial \tau_{0} / \partial \omega\right)\right\} \operatorname{det}\left\{{ }^{t} \tau_{1} \tau_{1}\right\}\right]^{1 / 2}\right|_{r=1} r^{n-1} d \omega d r \\
& \quad=\left.d \Sigma_{1} r^{n-1} d r\left[\operatorname{det}\left\{{ }^{t} \tau_{1} \tau_{1}\right\}\right]^{1 / 2}\right|_{r=1} .
\end{aligned}
$$

Remark. If we take $\tau_{1}(\omega, 1)$ as it is isometric, namely, $\left|\tau_{1}(\omega, 1) y\right|=|y|$, (4.2.10) is equal to $d \Sigma_{1} r^{n-1} d r$ because $\operatorname{det}\left\{{ }^{t} \tau_{1} \tau_{1}\right\}=1$.

We fix the above local coordinate $(\omega, r, y)$, that is, $\Omega$ seems $U \times \mathbb{R}_{+} \times Y$, $Y=\{|y|<L\}$. Now we check the properties (4.2.11-37) before calculating the rate.

$$
\begin{gathered}
\phi_{0}=-p_{m} t . \quad \text { (Definition.) } \\
\phi_{1}=-2^{-1}\left\langle\tau_{1}(\omega) y, \nabla^{2} p_{m}(\omega) \tau_{1}(\omega) y\right\rangle r^{m} t,
\end{gathered}
$$

where $\tau_{1}(\omega)=\tau_{1}(\omega, 1)$ and $\nabla^{2} p_{m}(\omega)=\left.\nabla^{2} p_{m}(\omega, r, y)\right|_{r=1, y=0}=\nabla^{2} p_{m}\left(\tau_{0}(\omega, 1)\right)$. (Definition.)

$$
\phi_{2}=-|y|^{2} r^{m} t . \quad \text { (Definition.) }
$$

(4.2.14) $\phi_{3}=-p_{m-1}(a) t+i \sigma^{1}\left((a-X)^{\sim}, \tanh (A(a) t / 2)(a-X)^{\sim}\right)$

$$
-2^{-1} \operatorname{Tr}(\log [\cosh (A(a) t / 2)])
$$

$$
\begin{aligned}
= & -p_{m-1}(\omega, r) t-\left\langle\tau_{1}(\omega) y, i J_{1} \tanh \left(A(\omega) r^{m-1} t / 2\right) \tau_{1}(\omega) y^{\prime}\right\rangle r^{r} \\
& -2^{-1} \operatorname{Tr}\left(\log \left[\cosh \left(A(\omega) r^{m-1} t / 2\right)\right]\right),
\end{aligned}
$$

$X=\tau(\omega, r, y), a=a(X)=\tau_{0}(\omega, r), A\left(\tau_{0}(\omega, r)\right)=r^{m-1} A\left(\tau_{0}(\omega, 1)\right)=r^{m-1} A(\omega)$ and $p_{m-1}(\omega, r)=p_{m-1}\left(\tau_{0}(\omega, r)\right)$. (Definition.)

(4.2.15) $\quad \phi_{4}=-\left\langle\tau_{1}(\omega) y, i J_{1} \tanh \left(A(\omega) r^{m-1} t / 2\right) \tau_{1}(\omega) y\right\rangle r . \quad$ (Definition.) 
(4.2.25) Let $\chi_{1}$ and $\chi_{2}$ be characteristic functions of $\Omega 1$ and $\Omega 2$. (Definition.)

$$
\left|\phi_{0}-\phi_{1}\right| \leqq-C|y| \phi_{2} \text { on } \Omega \text {. }
$$$$
\left|\exp \phi_{0}-\exp \phi_{1}\right| \leqq C|y| \exp \phi_{2} \text {. }
$$

$$
-\phi_{i} \leqq-C_{i j} \phi_{j}, \quad(i, j=0,1,2) .
$$$$
-\phi_{4} \geqq c|y|^{2} r \tanh \left(C r^{m-1} t\right) \text {. }
$$

$$
\exp \phi_{3}=\exp \phi_{4} \exp \left(-p_{m-1} t\right)\left[\operatorname{det}\left\{\cosh \left(A(\omega) r^{m-1} t / 2\right)\right\}\right]^{-1 / 2} \text {. }
$$

$$
\begin{aligned}
\operatorname{det} & \left\{\cosh \left(A(\omega) r^{m-1} t / 2\right)\right\} \\
= & \prod_{j=1}^{l}\left(\exp \left(-\lambda_{j} r^{m-1} t / 2\right)+\exp \left(\lambda_{j} r^{m-1} t / 2\right)\right)^{2} 2^{-2 l} \\
= & \exp \left(\operatorname{Tr}^{\sim} A(\omega) r^{m-1} t\right) \prod_{j=1}^{l}\left[\left(1+\exp \left(-\lambda_{j} r^{m-1} t\right)\right) / 2\right]^{2} .
\end{aligned}
$$

$$
\begin{aligned}
g & =\exp \left(-p_{m-1} t\right)\left[\operatorname{det}\left\{\cosh \left(A(\omega) r^{m-1} t / 2\right)\right\}\right]^{-1 / 2} \\
& =\exp \left(-p_{m-1} t-2^{-1} \operatorname{Tr}^{\sim} A(\omega) r^{m-1} t\right) \prod_{j=1}^{l}\left[\left(1+\exp \left(-\lambda_{j} r^{m-1} t\right)\right) / 2\right]^{-1} .
\end{aligned}
$$$$
g \leqq \exp \left(-\left(p_{m-1}(\omega)+2^{-1} \operatorname{Tr}^{\sim} A(\omega)\right) r^{m-1} t\right)
$$$$
\leqq \exp \left(-c r^{m-1} t\right) \text {. }
$$

according to (4.2.19).

(4.2.28) $\left|\phi_{4}-\phi_{1}\right| \leqq C t^{2} r^{2 m-1}|y|^{2} \leqq C \phi_{2} r^{m-1} t$, if $r \geqq 1$ and $r^{m-1} t \leqq 1$.

$$
\text { (Taylor's expansion in t.) }
$$

(4.2.29) $\left|\chi_{1}\left(\exp \phi_{4}-\exp \phi_{1}\right)\right| \leqq C r^{m-1} t \exp \left(c \phi_{2}\right)$, if $r \geqq 1$ and $r^{m-1} t \leqq 1$.

(4.2.30) $\left|\chi_{1}\left(\exp \phi_{3}-\exp \phi_{1}\right)\right| \leqq C r^{m-1} t \exp \left(c \phi_{2}\right)$, if $r \geqq 1$ and $r^{m-1} t \leqq 1$, by (4.2.24) and (4.2.29).

(4.2.31) $\left|\chi_{2} \exp \phi_{0}\right| \leqq C \chi_{2} \exp \left(c \phi_{2}\right) \leqq C \exp \left(c \phi_{2}-r^{m-1+2 \varepsilon} t\right)$.

(4.2.32) $\left|\chi_{2}\left(\exp \phi_{0}-\exp \phi_{1}\right)\right| \leqq C|y| \exp \left(c \phi_{2}\right)$, according to (4.2.17).

(4.2.33) We denote a function $h$ by $h^{\sim}$ when we change the variable $r^{m} t$ to $r^{m}$. $\lim _{t \rightarrow 0} \chi_{1}^{\tilde{1}}=0, \lim _{t \rightarrow 0} \chi_{2}=1$ and $\lim _{t \rightarrow 0} g^{\sim}=1$ almost everywhere in $\Omega$.

(4.2.34) We denote a function $h$ by $h^{*}$ when we change the variables $r^{m-1} t$ to $r^{m-1}$ and $y t^{-1 / 2(m-1)}$ to $y$.

$\lim _{t \rightarrow 0} \chi_{1}^{*}=1, \lim _{t \rightarrow 0} \chi_{2}^{*}=0$ almost everywhere in $U \times \mathbf{R}_{+} \times \mathbf{R}^{d}$, because 
$p_{m} r^{-m+1-2 \varepsilon} \leqq C|y|^{2} r^{1-2 \varepsilon}$ by (4.2.18), where $\chi_{1}^{*}=\chi_{2}^{*}=0$ out side $Y t^{-1 / 2(m-1)}$.

(4.2.35) If $\Phi$ is defined by $d x d \xi=\Phi r^{n-1} d r d y d \omega, \lim _{t \rightarrow 0} \Phi^{*}=\left.\Phi\right|_{y=0}$. (Refer to $(4.2 .7)$.)

$r^{k-1}|y|^{j} \exp \left(-c|y|^{2} r^{m}\right)$ belongs to $\mathbf{L}^{1}\left(\mathbf{R}_{+} \times Y\right)$ if $2 k-m(d+j)<0$, $(k>0)$.

$r^{k-1}|y|^{j} \exp \left(-c|y|^{2} r^{m}\right)$ belongs to $\mathbb{L}^{1}\left([0,1] \times \mathbb{R}^{d}\right)$ if $2 k-m(d+j)>0$.

\section{§4.3. Estimations}

Let $\chi_{1}$ and $\chi_{2}$ be characteristic functions of $\Omega 1$ and $\Omega 2$, respectively. We define $I(W)$ and $J(W)$ by (4.3.1-2), where we use the notation of Section 4.2.

$$
\begin{aligned}
& I(W)=\int_{W} \chi_{1} \exp \phi_{3} d x d \xi . \\
& J(W)=\int_{W} \chi_{2} \exp \phi_{0} d x d \xi .
\end{aligned}
$$

We divide $\Omega$ into three parts $\Omega a, \Omega b$ and $\Omega c$, depending on $t$, such that $\Omega a$ $=\left\{r^{m-1} t>1\right.$ and $\left.r>1\right\}, \Omega b=\left\{t<r^{m-1} t \leqq 1\right\}$ and $\Omega c=\{r \leqq 1\}$, and we denote the union of $\Omega a$ and $\Omega b$ by $\Omega a b$. It is clear that $I(\Omega c)=O(1)$ and $J(\Omega c)=O(1)$ as $t$ tends to zero.

(*) In the case that $2 n-m d>0, I(\Omega a b) \sim c t^{-(n-d / 2) /(m-1)}$ and $J(\Omega a b)$ $=o\left(t^{-(n-d / 2) /(m-1)}\right)$.

By (4.2.31), $J(\Omega a b) \leqq C \int_{\Omega a b} \exp \left(c\left(\phi_{2}-r^{m-1+2 \varepsilon} t\right)\right) r^{n-1} d w d r d y$. Changing variables $r t^{1 /(m-1+2 \varepsilon)}$ to $r$ and $y t^{(2 \varepsilon-1) / 2(m-1+2 \varepsilon)}$ to $\mathrm{y}^{\prime}$,

$$
\begin{aligned}
& J(\Omega a b) \\
& \leqq C \int_{D} \exp \left(c\left(\phi_{2}(1)-r^{m-1+2 \varepsilon}\right)\right) r^{n-1} d w d r d y t^{-(n-(1-2 \varepsilon) d / 2) /(m-1+2 \varepsilon)},
\end{aligned}
$$

where $D=U \times\left[t^{1 /(m-1+2 \varepsilon)},+\infty\right) \times Y t^{(2 \varepsilon-1) / 2(m-1+2 \varepsilon)}$ and $\phi_{2}(1)=\left.\phi_{2}\right|_{t=1}$. By (4.2.37), $r^{n-1} \exp \left(c\left(\phi_{2}(1)-r^{m-1+2 \varepsilon}\right)\right)$ belongs to $\mathbf{L}^{1}\left(U \times \mathbf{R}_{+} \times \mathbf{R}^{d}\right)$ since $2 n-m d$ $>0$. Therefore we get $(4.3 .3)$ because $(n-(1-2 \varepsilon) d / 2) /(m-1+2 \varepsilon)<(n-d / 2) /$ $(m-1)$.

$$
\begin{aligned}
J(\Omega a b) & =O\left(t^{-(n-(1-2 \varepsilon) d / 2) /(m-1+2 \varepsilon)}\right) \\
& =O\left(t^{-(n-d / 2) /(m-1)}\right) .
\end{aligned}
$$

On the other hand we get (4.3.4) about $I(\Omega a b)$ changing variables $r t^{1 /(m-1)}$ to $r$ and $y t^{-1 / 2(m-1)}$ to $y$. 


$$
I(\Omega a b)=\int_{D} \chi_{1}^{*} \Phi^{*} r^{n-1} \exp \phi_{3}(1) d w d r d y t^{-(n-d / 2) /(m-1)},
$$

where $D=U \times\left[t^{1 /(m-1)},+\infty\right) \times Y t^{-1 / 2(m-1)}$ and $\phi_{3}(1)=\left.\phi_{3}\right|_{t=1}$. By (4.2.26), the integrand of (4.3.4) is bounded by $C r^{n-1} \exp \left(-c|y|^{2} r \tanh r^{m-1}-c r^{m-1}\right)$, which belongs to $\boldsymbol{L}^{1}\left(U \times \mathbf{R}_{+} \times \mathbb{R}^{d}\right)$ by (4.2.37). Using Lebesgue's theorem we get (4.3.5) by (4.2.35).

$$
I(\Omega a b)=\left\{\int_{D} \exp \phi_{3}(1) d \Sigma_{1} r^{n-1} d r\left[\operatorname{det}\left\{{ }^{t} \tau_{1} \tau_{1}\right\}\right]^{1 / 2} d y+o(1)\right\} t^{-(n-d / 2) /(m-1)},
$$

where $D=U \times \mathbf{R}_{+} \times \mathbf{R}^{d}$.

(*) In the case that $2 n-m d=0, I(\Omega)+J(\Omega)=\int_{\Omega b} \exp \phi_{1} d x d \xi+O\left(t^{-n / m}\right)$.

In the same way as in the case that $2 n-m d>0, I(\Omega a)=O\left(t^{-(n-d / 2) /(m-1)}\right)$ $=O\left(t^{-n / m}\right)$ because $2 n-m d=0$. Let us put $Z_{1}=\chi_{1}\left(\exp \phi_{3}-\exp \phi_{1}\right)$. Then $\left|Z_{1}\right| \leqq C r^{m-1} t \exp \left(c \phi_{2}\right)$ on $\Omega b$ by (4.2.30). So we get (4.3.6) by changing variable $r t^{1 /(m-1)}$ to $r$ and $y t^{-1 / 2(m-1)}$ to $y$.

$$
\int_{\Omega b}\left|Z_{1}\right| d x d \xi \leqq C \int_{D} r^{m-1+n-1} \exp \left(c \phi_{2}(1)\right) d w d r d y t^{-(n-d / 2) /(m-1)},
$$

where $D=U \times\left[t^{1 /(m-1)}, 1\right] \times Y t^{-1 / 2(m-1)}$ and $\phi_{2}(1)=\left.\phi_{2}\right|_{t=1}$. Since $m-1+n$ $-m d / 2=m-1>0$, we can use (4.2.37).

$$
\int_{\Omega b}\left|Z_{1}\right| d x d \xi \leqq C \int_{D} r^{m+n-2} \exp \left(c \phi_{2}(1)\right) d w d r d y t^{-(n-d / 2) /(m-1)},
$$

where $D=U \times[0,1] \times \mathbf{R}^{d}$. Therefore we get (4.3.8) because $2 n-m d=0$.

$$
\int_{\Omega b} Z_{1} d x d \xi=O\left(t^{-n / m}\right)
$$

Therefore we get (4.3.9).

$$
I(\Omega)=\int_{\Omega b} \chi_{1} \exp \phi_{1} d x d \xi+O\left(t^{-n / m}\right) .
$$

According to (4.2.17) $Z_{2}=\chi_{2}\left(\exp \phi_{0}-\exp \phi_{1}\right)$ is bounded by $C|y| \exp c \phi_{2}$ on $\Omega$. Changing variable $r t^{1 / m}$ to $r$ we get (4.3.10) by (4.2.36).

$$
\int_{\Omega b}\left|Z_{2}\right| d x d \xi \leqq C \int_{D}|y| r^{n-1} \exp \left(c \phi_{2}(1)\right) d w d r d y t^{-n / m}=O\left(t^{-n / m}\right),
$$

where $D=U \times\left[t^{1 / m}, t^{-1 / m(m-1)}\right] \times Y$. On the other hand we get (4.3.11) by (4.2.31) and by changing variables $r t^{1 /(m-1+2 \varepsilon)}$ to $r$ and $y t^{(2 \varepsilon-1) / 2(m-1+2 \varepsilon)}$ to $y$. 


$$
\begin{aligned}
& |J(\Omega a)| \leqq C \int_{D} r^{n-1} \exp \left(c\left(\phi_{2}-r^{m-1+2 \varepsilon} t\right)\right) d w d r d y \\
& \quad=\int_{D^{\prime}} r^{n-1} \exp \left(c\left(\phi_{2}(1)-r^{m-1+2 \varepsilon}\right)\right) d w d r d y t^{-(n-(1-2 \varepsilon) d / 2) /(m-1+2 \varepsilon)},
\end{aligned}
$$

where $\quad D=U \times\left[t^{-1 /(m-1)},+\infty\right) \times Y$ and $D^{\prime}=U \times\left[t^{-2 \varepsilon /(m-1)(m-1+2 \varepsilon)},+\infty\right) \times$ $Y t^{(2 \varepsilon-1) / 2(m-1+2 \varepsilon)}$. Since $\exp \left(c\left(\phi_{2}(1)-r^{m-1+2 \varepsilon}\right)\right)$ belongs to $\mathbb{L}^{1}(U \times[1,+\infty)$ $\left.\times \mathbb{R}^{d}\right)$ and $(n-d / 2) /(m-1)-(n-(1-2 \varepsilon) d / 2) /(m-1+2 \varepsilon)=2 \varepsilon(n-m d / 2) /$ $(m-1)(m-1+2 \varepsilon)=0$, we get (4.3.12).

$$
J(\Omega a)=O\left(t^{-(n-(1-2 \varepsilon) d / 2) /(m-1+2 \varepsilon)}\right)=O\left(t^{-n / m}\right) .
$$

Therefore (4.3.13) holds.

$$
J(\Omega)=\int_{\Omega b} \chi_{2} \exp \phi_{1} d x d \xi+O\left(t^{-n / m}\right)
$$

Since $\int_{\Omega b}\left(1-\chi_{1}-\chi_{2}\right) \exp \phi_{1} d x d \xi=O(1)$, the sum with $I(\Omega)$ comes to (4.3.14).

$$
I(\Omega)+J(\Omega)=\int_{\Omega b} \exp \phi_{1} d x d \xi+O\left(t^{-n / m}\right) .
$$

(*) In the case that $2 n-m d<0, I(\Omega)+J(\Omega) \sim \int_{\Omega} \exp \phi_{0}(1) d x d \xi t^{-n / m}$.

For $I(\Omega a)$, we get that $I(\Omega a)=O\left(t^{-(n-d / 2) /(m-1)}\right)$ in the same way as in the case that $2 n-m d>0$. Changing variable $r t^{1 / m}$ to $r$, we get (4.3.15) for $I(\Omega b)$ and $J(\Omega a b)$ because $\Phi$ is independent of $r$.

$$
I(\Omega b)=\int_{D} g^{\sim} \chi_{1}^{\sim} \Phi r^{n-1} \exp \left(\phi_{4}\left(t^{1 / m}\right) t^{-1 / m}\right) d w d r d y^{-n / m}
$$

and

$$
J(\Omega a b)=\int_{D^{\prime}} \chi_{2} \Phi r^{n-1} \exp \left(\phi_{0}(1)\right) d w d r d y t^{-n / m},
$$

where $D=U \times\left[t^{1 / m}, t^{-1 / m(m-1)}\right] \times Y$ and $D^{\prime}=U \times\left[t^{1 / m},+\infty\right) \times Y$. By (4.2.27) and (4.2.31) the integrands are bounded by $C r^{n-1} \exp \left(c\left(\phi_{2}(1)\right)\right.$, which belongs to $\boldsymbol{L}^{1}(U \times[0,+\infty) \times Y)$ by (4.2.36). Using (4.2.33) and Lebesgue's theorem we get (4.3.16).

$$
I(\Omega b)=o\left(t^{-n / m}\right)
$$

and

$$
\begin{aligned}
J(\Omega a b) & =\left(\int_{D^{\prime}} \exp \phi_{0}(1) \Phi r^{n-1} d w d r d y+o(1)\right) t^{-n / m} \\
& =\left(\int_{\Omega} \exp \phi_{0}(1) d x d \xi+o(1)\right) t^{-n / m}
\end{aligned}
$$

We conclude the following proposition by the above discussion. 


\section{Proposition 4.2.}

$$
\begin{aligned}
& I(\Omega)+J(\Omega)=\left(\int_{\Omega} \exp \phi_{0}(1) d x d \xi+o(1)\right) t^{-n / m}, \quad \text { if } \quad 2 n-m d<0, \\
& =\int_{\Omega b} \exp \phi_{1} d x d \xi+O\left(t^{-n / m}\right), \quad \text { if } 2 n-m d=0
\end{aligned}
$$

or

$$
\begin{aligned}
= & \left(\int_{D} r^{n-1} \exp \phi_{3}(1) d \Sigma_{1} d r\left[\operatorname{det}\left\{{ }^{t} \tau_{1} \tau_{1}\right\}\right]^{1 / 2} d y\right. \\
& +o(1)) t^{-(n-d / 2) /(m-1)}, \quad \text { if } 2 n-m d>0,
\end{aligned}
$$

where $D=U \times \mathbf{R}_{+} \times \mathbf{R}^{d}$.

Proposition 4.3. If $2 n-m d=0$,

$$
\begin{aligned}
& \int_{\Omega b} \exp \phi_{1} d x d \xi \\
& \quad=\left\{(2 \pi)^{d / 2} \int_{U}\left[\operatorname{det} H_{+}\right]^{-1 / 2} d \Sigma_{1}[m(m-1)]^{-1} \log t^{-1}+O(1)\right\} t^{-n / m},
\end{aligned}
$$

where $U=\Sigma_{1}$ is the intersection of $\Sigma$ and $S^{*} \mathbf{R}^{n}, H=\nabla^{2} p_{m}, H_{+}$is the restriction of $H$ on the range of $H$ and $\Omega b=U \times\left[1, t^{-1 /(m-1)}\right] \times Y$.

Proof.

$$
\int_{\Omega b} \exp \phi_{1} d x d \xi=\int_{\Omega b}\left(\exp \phi_{1}\right) \Phi r^{n-1} d w d r d y
$$

Changing variable $r t^{1 / m}$ to $r$, this is equal to

$$
\int_{D}\left(\exp \phi_{1}(1)\right) \Phi r^{n-1} d w d r d y t^{-n / m},
$$

where $D=U \times\left[t^{1 / m}, t^{-1 / m(m-1)}\right] \times Y$. Dividing the domain $D$ into two parts $D 1$ and $D 1^{\prime}$ such that $D 1=U \times\left[1, t^{-1 / m(m-1)}\right] \times Y$ and $D 1^{\prime}=U \times\left[t^{1 / m}, 1\right) \times Y$, (4.3.20) is equal to

$$
\begin{aligned}
& \int_{D 1}\left(\exp \phi_{1}(1)\right) \Phi r^{n-1} d w d r d y t^{-n / m} \\
& +\int_{D 1^{\prime}}\left(\exp \phi_{1}(1)\right) \Phi r^{n-1} d w d r d y t^{-n / m}
\end{aligned}
$$

Since the second term is $O\left(t^{-n / m}\right)$, we consider the first term, which is also divided into a sum of two terms. 
(4.3.22) $\quad t^{n / m}[$ the first term of (4.3.21)]

$$
=\int_{D 1}\left(\exp \phi_{1}(1)\right) \Phi_{0} r^{n-1} d w d r d y+\int_{D 1}\left(\exp \phi_{1}(1)\right)\left(\Phi-\Phi_{0}\right) r^{n-1} d w d r d y
$$

where $\Phi_{0}=\left.\Phi\right|_{y=0}$.

Since $\left|\Phi-\Phi_{0}\right| \leqq C|y|$, the second term of (4.3.22) is bounded according to (4.2.36). So we consider also the first term. Since $\exp \phi_{1}(1) \leqq \exp \left(-c\left(|y|^{2}\right.\right.$ $\left.\left.+r^{m}\right)\right)$ on $U \times[1,+\infty) \times\left(\mathbb{R}^{d} \backslash Y\right)$, it suffices to calculate $M=\int_{D}\left(\exp \phi_{1}(1)\right) \Phi_{0} r^{n-1}$ $d w d r d y$, where $D=U \times\left[1, t^{-1 / m(m-1)}\right] \times \mathbb{R}^{d}$, because it becomes to

(4.3.23) [the first term of (4.3.22)] $=M+O(1)$.

$$
\begin{aligned}
M & =\int_{D} \exp \left(-\left\langle\tau_{1}(\omega) y,\left(\nabla^{2} p_{m} / 2\right) \tau_{1}(\omega) y\right\rangle r^{m}\right) d \Sigma_{1} r^{n-1} d r\left[\operatorname{det}\left\{\tau_{1} \tau_{1}\right\}\right]^{1 / 2} d y \\
& =\int_{D^{\prime}}(\pi)^{d / 2}\left[\operatorname{det}\left\{H_{+} / 2\right\}\right]^{-1 / 2} r^{n-m d / 2-1} d \Sigma_{1} d r \\
& \text { where } D^{\prime}=U \times\left[1, t^{-1 / m(m-1)}\right] \\
& =(2 \pi)^{d / 2} \int_{U}\left[\operatorname{det} H_{+}\right]^{-1 / 2} d \Sigma_{1}[m(m-1)]^{-1} \log t^{-1}
\end{aligned}
$$

because $n-m d / 2=0$.

q.e.d.

Proposition 4.4. If $2 n-m d>0$,

$$
\begin{aligned}
\int_{D} & \left(\exp \phi_{3}(1)\right)\left[\operatorname{det}\left\{{ }^{t} \tau_{1} \tau_{1}\right\}\right]^{1 / 2} d y, \text { where } D=\mathbb{R}^{d} \\
= & (2 \pi)^{d / 2} \exp \left(-p_{m-1}\right)\left[\operatorname{det}\left\{H_{+}(\omega) r^{m-1}\right\}\right]^{-1 / 2} \\
& \times\left[\operatorname{det}\left\{\left(A(\omega) r^{m-1} / 2\right)^{-1} \sinh \left(A(\omega) r^{m-1} / 2\right)\right\}\right]^{-1 / 2} r^{-d / 2},
\end{aligned}
$$

and

$$
\begin{aligned}
\int_{D} & \left(\exp \phi_{3}(1)\right) r^{n-1} d \Sigma_{1} d r\left[\operatorname{det}\left\{{ }^{t} \tau_{1} \tau_{1}\right\}\right]^{1 / 2} d y, \text { where } D=U \times \mathbb{R}_{+} \times \mathbb{R}^{d} \\
= & (2 \pi)^{d / 2} \int_{\Sigma} \exp \left(-p_{m-1}\right)\left[\operatorname{det}\left\{(A / 2)^{-1} \sinh (A / 2)\right\}\right]^{-1 / 2} \\
& \times\left[\operatorname{det} H_{+}\right]^{-1 / 2} d \Sigma_{1} r^{n-d / 2-1} d r
\end{aligned}
$$

Proof.

$$
\begin{aligned}
K & =\int_{D}\left(\exp \phi_{3}(1)\right)\left[\operatorname{det}\left\{{ }^{t} \tau_{1} \tau_{1}\right\}\right]^{1 / 2} d y, \text { where } D=\mathbb{R}^{d} \\
& =\left(\exp \left(-p_{m-1}\right)\right)\left[\operatorname{det}\left\{\cosh \left(A(\omega) r^{m-1} / 2\right)\right\}\right]^{-1 / 2} K_{0}
\end{aligned}
$$

where

$$
\begin{aligned}
K_{0}= & \int_{D} \exp \left(-\left\langle\tau_{1}(\omega) y, i J_{1} \tanh \left(A(\omega) r^{m-1} / 2\right) \tau_{1}(\omega) y\right\rangle r\right) \\
& \times\left[\operatorname{det}\left\{{ }^{t} \tau_{1} \tau_{1}\right\}\right]^{1 / 2} d y .
\end{aligned}
$$

By the properties of Hamilton matrix, there exists a base $(e, \underline{e}, c)$ on range of 
$H=$ range of $\tau_{1}(\omega)$ such that by the coordinate $x, \underline{x}$ and $z$ with respect to $(e, \underline{e}, c)$, satisfying $\tau_{1}(\omega) y=x e+\underline{x e}+z c$,

$$
\begin{aligned}
& -\left\langle\tau_{1}(\omega) y, i J_{1} \tanh \left(A(\omega) r^{m-1} / 2\right) \tau_{1}(\omega) y^{\cdot}\right\rangle \\
& =\sum G\left(\lambda_{j}\right)\left(2\left|x_{j}\right|^{2}\right)+\sum\left|z_{k}\right|^{2},
\end{aligned}
$$

where $G\left(\lambda_{j}\right)=\left(\tanh \lambda_{j}\right) r$ with eigenvalues $\lambda_{j}$ of $A(\omega) r^{m-1} / 2$. (Refer to Appendix.)

Denoting the mapping from $y$ to $(x, \underline{x}, z)$ by $\Psi$ we get

$$
\begin{aligned}
K_{0} & =\int \exp \left(\sum G\left(\lambda_{j}\right)\left(2\left|x_{j}\right|^{2}\right)+\sum\left|z_{j}\right|^{2}\right)[\operatorname{det} \Psi]^{-1}\left[\operatorname{det}\left\{{ }^{t} \tau_{1} \tau_{1}\right\}\right]^{1 / 2} d x d \underline{x} d z \\
& =\pi^{d / 2} \Pi G\left(\lambda_{j}\right)^{-1}[\operatorname{det} \Psi]^{-1}\left[\operatorname{det}\left\{{ }^{t} \tau_{1} \tau_{1}\right\}\right]^{1 / 2} r^{-d / 2} \\
& =\pi^{d / 2}\left[\left|\operatorname{det}\left\{\tanh \left(A(\omega) r^{m-1} / 2\right)\right\}\right|\right]^{-1 / 2}[\operatorname{det} \Psi]^{-1}\left[\operatorname{det}\left\{{ }^{t} \tau_{1} \tau_{1}\right\}\right]^{1 / 2} r^{-d / 2}
\end{aligned}
$$

Therefore we get

$$
\begin{aligned}
K= & \pi^{d / 2}\left(\exp \left(-p_{m-1}\right)\right)\left[\left|\operatorname{det}\left\{\sinh \left(A(\omega) r^{m-1} / 2\right)\right\}\right|\right]^{-1 / 2} \\
& \times[\operatorname{det} \Psi]^{-1}\left[\operatorname{det}\left\{\tau_{1} \tau_{1}\right\}\right]^{1 / 2} r^{-d / 2}
\end{aligned}
$$

On the other hand we know that

$$
\operatorname{det}\left\{{ }^{t} \tau_{1}\left(H_{+} / 2\right) \tau_{1}\right\}=\operatorname{det}\left\{H_{+} / 2\right\} \operatorname{det}\left\{{ }^{t} \tau_{1} \tau_{1}\right\}=\prod \lambda_{j}^{2}[\operatorname{det} \Psi]^{2}
$$

so that we get the conclusion (4.3.25). Integrating (4.3.25) by $r^{n-1} d \Sigma_{1} d r$ we get

$$
\begin{aligned}
\int_{D}( & \left.\exp \phi_{3}(1)\right) r^{n-1} d \Sigma_{1} d r\left[\operatorname{det}\left\{{ }^{t} \tau_{1} \tau_{1}\right\}\right]^{1 / 2} d y, \text { where } D=U \times \mathbf{R}_{+} \times \mathbf{R}^{d} \\
= & \pi^{d / 2} \int_{D^{\prime}}\left(\exp \left(-p_{m-1}(\omega)\right) r^{m-1}\right)\left[\operatorname{det}\left\{H_{+}(\omega) r^{m-1} / 2\right\}\right]^{-1 / 2} \\
& \left.\times\left[\operatorname{det}\left\{A(\omega) r^{m-1} / 2\right)^{-1} \sinh \left(A(\omega) r^{m-1} / 2\right)\right\}\right]^{-1 / 2} d \Sigma_{1} r^{n-d / 2-1} d r \\
= & (2 \pi)^{d / 2} \int_{D^{\prime}}\left(\exp \left(-p_{m-1}\right)\right)\left[\operatorname{det}\left\{(A / 2)^{-1} \sinh (A / 2)\right\}\right]^{-1 / 2} \\
& \times\left[\operatorname{det} H_{+}\right]^{-1 / 2} d \Sigma_{1} r^{n-d / 2-1} d r
\end{aligned}
$$

where $D^{\prime}=U \times \mathbf{R}_{+}$.

q.e.d.

(*) About a density on $\Sigma$.

(4.3.33) $\left[\operatorname{det} H_{+}\right]^{-1 / 2} d \Sigma_{1} r^{n-d / 2-1} d r=\left[\operatorname{det}\left\{H_{+}(\omega) r^{m}\right\}\right]^{-1 / 2} d \Sigma_{1} r^{n-1} d r$.

This is an induced density $d \Sigma$ on $\Sigma$ by $p_{m}$ and the canonical density $d \Omega=$ $\left|d x_{1} \wedge d \xi_{1} \cdots d x_{n} \wedge d \xi_{n}\right|$. In fact it is defined as followings. Let $(x, y)$ a local coordinate of $\Omega$ such that $\Sigma=\{y=0\},\left(x=\left(x_{1}, \ldots, x_{2 n-d}\right)\right.$ and $\left.y=\left(y_{1}, \ldots, y_{d}\right)\right)$. The canonical density is written as $d \Omega=G d x d y$ and $d \Sigma$ is defined by $d \Sigma=$ 
$\left[\operatorname{det}\left\{\partial_{y}^{2} p_{m}\right\}\right]^{-1 / 2} G d x$. If we take $(x, y)$ as $x=(r, \omega)$ and $y$ is orthogonal to $\Sigma$, $d \Sigma$ is given by (4.3.33). Therefore we get (4.3.34) when $2 n-m d>0$.

$$
\begin{aligned}
& \int_{\Omega} e(x, \xi) d x d \xi=\left\{(2 \pi)^{d / 2} \int_{\Sigma} \exp \left(-p_{m-1}\right)\right. \\
& \left.\quad \times\left[\operatorname{det}\left\{(A / 2)^{-1} \sinh (A / 2)\right\}\right]^{-1 / 2} d \Sigma+o(1)\right\} t^{-(n-d / 2) /(m-1)},
\end{aligned}
$$

where $d \Sigma=\left[\operatorname{det} H_{+}\right]^{-1 / 2} d \Sigma_{1} r^{n-d / 2-1} d r$.

When $2 n-m d=0, d \Sigma$ may seem a density $d \Sigma_{s}$ on $\Sigma_{s}$ the projection of $\Sigma$ into the spherical bundle $S^{*} U$ as (4.3.35) because functions on $\Sigma_{s}$ may be identified with homogeneous functions on $\Sigma$ of order zero.

$$
\int_{\Sigma_{s}} f d \Sigma_{s}=(m-1) \int_{\Sigma} f h \exp (-h) d \Sigma,
$$

where $h$ may be any positive function on $\Sigma$ with homogeneous order $m-1$. Since $\omega$ of $(\omega, r, y)$ may seem a local coordinate of $\Sigma_{s}$,

$$
\begin{aligned}
& =\left\{(m-1) \int_{0}^{+\infty} h(\omega) r^{m-1} \exp \left(-h(\omega) r^{m-1}\right)\left[\operatorname{det} H_{+}\right]^{-1 / 2} r^{n-d / 2-1} d r\right\} d \Sigma_{1} \\
& =\left[\operatorname{det} H_{+}\right]^{-1 / 2} d \Sigma_{1} .
\end{aligned}
$$

Therefore when $2 n-m d=0$, we get (4.3.37), and also (4.3.38) if we want to leave it an integral on $\Sigma$ using a function given a-priori there, $p_{m-1}+2^{-1} \operatorname{Tr} \sim A$.

$$
\begin{aligned}
\int_{W} e(x, \xi) d x d \xi= & \left\{(2 \pi)^{d / 2} m^{-1}(m-1)^{-1} \int d \Sigma_{s} \log t^{-1}+O(1)\right\} t^{-n / m} \\
= & \left\{(2 \pi)^{d / 2} m^{-1} \int\left(p_{m-1}+2^{-1} \operatorname{Tr} \sim A\right)\right. \\
& \left.\times \exp \left(-\left(p_{m-1}+2^{-1} \operatorname{Tr}^{\sim} A\right)\right) d \Sigma \log t^{-1}+O(1)\right\} t^{-n / m}
\end{aligned}
$$

Thus we conclude Proposition 4.5.

Proposition 4.5. Let $\Omega$ be a conic neighborhood of $X$ belonging to $\Sigma$.

$$
\begin{aligned}
\int_{\Omega} & e(x, \xi) d x d \xi \\
& =\left\{\int_{\Omega} \exp \left(-p_{m}\right) d x d \xi+o(1)\right\} t^{-n / m}, \quad \text { if } 2 n-m d<0, \\
& =\left\{(2 \pi)^{d / 2} m^{-1}(m-1)^{-1} \int_{\Sigma_{s}} d \Sigma_{s} \log t^{-1}+O(1)\right\} t^{-n / m}, \text { if } 2 n-m d=0
\end{aligned}
$$

or 


$$
\begin{aligned}
= & \left\{(2 \pi)^{d / 2} \int_{\Sigma} \exp \left(-p_{m-1}\right)\left[\operatorname{det}\left\{(A / 2)^{-1} \sinh (A / 2)\right\}\right]^{-1 / 2} d \Sigma\right. \\
& +o(1)\} t^{-(n-d / 2) /(m-1)}, \quad \text { if } 2 n-m d>0 .
\end{aligned}
$$

Remark. See the discussion before the proposition for $d \Sigma$ and $d \Sigma_{s}$.

(*) By this conclusion it is easy to obtain Theorem 4.2 noting the dimension of each connected component of the characteristic set $\Sigma$.

\section{§4.4. Reconsideration about General Cases}

In the results of Theorem 4.2 it is difficult to rewrite the second and third cases, namely, the case that $2 n-m d \geqq 0$, into a simple statement of general cases, because $d$ the codimension of the characteristic set $\Sigma$ and 2 (of $n-d / 2$ or $2 n-m d)$ the vanishing order of $p_{m}$ at $\Sigma$ reflect complicatedly on the rate. However the first case, namely, the case that $2 n-m d<0$, may be caught as $\exp \left(-p_{m}\right)$ is integrable on $T^{*} M$, that is, as the measure of $\left\{p_{m} \leqq 1\right\}$ is finite. Then to obtain the same result we do not need the condition that $p_{m}$ vanishes exactly double on the characteristic set $\Sigma$.

Proposition 4.6. Let $X$ be a point of the characteristic set $\Sigma$ and $\Omega$ be a small conic neighborhood of $X$. Then we get (4.4.1) as t tends to zero under Condition $(A)$ if $\int_{\Omega} \exp \left(-p_{m}\right) d x d \xi$ is finite.

$$
\int_{\Omega} e(x, \xi) d x d \xi=\left\{\int_{\Omega} \exp \left(-p_{m}\right) d x d \xi+o(1)\right\} t^{-n / m} .
$$

Proof. By Corollary of Theorem 1 we calculate $\int_{\Omega 1} \exp \phi_{1} d x d \xi$ and $\int_{\Omega 2} \exp \left(-t p_{m}\right) d x d \xi$, where $\Omega 1$ and $\Omega 2$ are restricted on $\Omega$. For $\int_{\Omega 2} \exp \left(-t p_{m}\right)$ $\times d x d \xi$ we get immediately (4.4.2) changing variable $\xi t^{1 / m}$ to $\xi$.

$$
\int_{\Omega 2} \exp \left(-t p_{m}\right) d x d \xi=\left\{\int_{\Omega} \exp \left(-p_{m}\right) d x d \xi+o(1)\right\} t^{-n / m} .
$$

On the other hand the main part of $\phi_{1}$ is $-\Phi_{1}$, which may be changed by $-\Phi_{2}$ according to Proposition 1.17. (Refer to Section 1.6 for notations.) Therefore we get

$$
\left|\exp \phi_{1}\right| \leqq C \exp \left(-\Phi_{2}-c\langle\xi\rangle^{m-1} t\right) \leqq C \exp \left(-\Phi_{2}\right) .
$$

We consider a new variable $Y=X+\iota \chi h$. Then $\Phi_{2}(X)=p_{m}(Y)$ and the Jacobian of the transformation is bounded for a sufficiently large $\langle\xi\rangle$ because we know 
(1.6.9) and $\delta<1 / 6$. More precisely it goes to identity, and $Y$ tends to $X$ as $\langle\xi\rangle$ tends to infinity. Therefore domain $\Omega 1$ is not essentially changed, that is, $\Omega 1$ is contained in $\Omega 1^{\prime}=\left\{Y=(y, \eta) ; p_{m}(Y) \leqq C\langle\eta\rangle^{m-1+2 \varepsilon}\right\}$. Thus we get

$$
\left|\int_{\Omega 1} \exp \phi_{1} d x d \xi\right| \leqq C \int_{\Omega 1^{\prime}} \exp \left(-p_{m}(Y)\right) d y d \eta \text {. }
$$

Changing variable $\eta t^{1 / m}$ to $\eta$ the right hand side has the rate $o(1) t^{-n / m}$ as $t$ tends to zero. Therefore we conclude the result of Proposition 4.6.

q.e.d.

(*) It is clear for Proposition 4.6 to imply Theorem 4.3.

(*) We give notes about Remark after Theorem 4.1.

It is clear by the process of proofs that $\phi^{\prime}$ satisfies Proposition 1.25 as well as $\phi$, where $\phi_{0}$ should be replaced to $\phi_{0}^{\prime}$ in which $\psi_{1}$ and $\psi_{1}^{1}$ of $\phi_{0}$ had been replaced to the following $\psi_{1}^{\prime}$ and $\psi_{1}^{1^{\prime}}$.

$$
\begin{aligned}
& \psi_{1}^{\prime}=\psi\left(p_{m} \Xi^{1-m-2 \varepsilon}\right) \psi\left(t \Xi^{m-1-\delta}\right) . \\
& \psi_{1}^{\prime}=\psi\left(p_{m} \Xi^{1-m-2 \varepsilon}\right) .
\end{aligned}
$$

Meanwhile by definition (refer to $(0.2 .3-7)$ ), the difference of $\phi$ and $\phi^{\prime}$ is

$$
\phi-\phi^{\prime}=\left(\psi_{1}-\psi_{1}^{\prime}\right)\left(\phi_{1}-\phi_{2}^{\prime}\right)+\left(1-\psi_{1}\right)\left(\Xi^{m-1}-\langle\xi\rangle^{m-1}\right) t .
$$

By (1.4.3), $\left|\phi_{1}-\phi_{2}^{\prime}\right|$ is bounded as

$$
\left|\phi_{1}-\phi_{2}^{\prime}\right| \leqq C\left\{t\langle\xi\rangle^{m-1}+\left(t\langle\xi\rangle^{m-1+\varepsilon}\right)^{2}\right\}\left(1+t\langle\xi\rangle^{m-1}\right)^{d^{\prime}} .
$$

Since $\psi_{1}-\psi_{1}^{\prime}=0$ and $1-\psi_{1}=0$ if $p_{m}\langle\xi\rangle^{1-m-2 \varepsilon} \leqq \min \left(1, c^{\prime-1}\right)$, we get

$$
\begin{aligned}
\left|\phi-\phi^{\prime}\right| & \leqq C\langle\xi\rangle^{-2 \varepsilon}\left(1+\phi_{0}\right)^{d} \\
& \leqq C\langle\xi\rangle^{-2 \varepsilon}\left(1+\phi_{0}^{\prime}\right)^{d},
\end{aligned}
$$

noting (1.6.28). Now we prove that $\exp \phi$ and $\exp \phi^{\prime}$ are equal to each other asymptotically. We divide the domain to two parts. Let $w(t, x, \xi)$ be such a characteristic function that $w=1$ if $\phi \geqq \phi^{\prime}$ and $w=0$ if $\phi<\phi^{\prime}$. Then we get

$$
w\left(\exp \phi-\exp \phi^{\prime}\right)=\left\{1-\exp \left(\phi^{\prime}-\phi\right)\right\} w \exp \phi,
$$

and

$$
1-\exp \left(\phi^{\prime}-\phi\right)=\left(\phi^{\prime}-\phi\right) \int_{0}^{1} \exp \left\{\left(\phi^{\prime}-\phi\right) \theta\right\} d \theta
$$

By (4.4.7), $\left|1-\exp \left(\phi^{\prime}-\phi\right)\right| \leqq C\langle\xi\rangle^{-2 \varepsilon}\left(1+\phi_{0}\right)^{d}$. Therefore by the same procedure as the proof of Theorem 4.1 ,

$$
(1+o(1)) \int_{T^{*} U} w \exp \phi d x d \xi=\int_{T^{*} U} w \exp \phi^{\prime} d x d \xi .
$$


In the same way we get

$$
\int_{T^{*} U}(1-w) \exp \phi d x d \xi=(1+o(1)) \int_{T^{*} U}(1-w) \exp \phi^{\prime} d x d \xi
$$

These imply that

$$
\int_{T^{*} U} \exp \phi d x d \xi=(1+o(1)) \int_{T^{*} U} \exp \phi^{\prime} d x d \xi
$$

\section{Appendix}

\section{§ A.1. Hamilton (Fundamental) Matrix}

Here we state some properties about Hamilton matrices and prove Proposition 1.2.

Let $H$ be a real symmetric matrix on $X=\mathrm{C}^{2 n}, J$ be a real unitary matrix such that $J^{2}=-I$ through this section. We also put $A=i J H$. ( , ) stands for the canonical inner product of $X$ and $\langle$,$\rangle is a bilinear form such that (x, y)$ $=\langle x, \underline{y}\rangle$, where $\underline{y}=y^{\mathrm{conj}}$ is the complex conjugate of $y$.

(*) At first we assume that $H$ is non-negative. We denote the range of $H$ by $Y$ and the kernel of $H$ by $N$. Then $X=Y \oplus N$ is an orthogonal decomposition of $X$ because $H$ is symmetric. We subdivide $Y$ as $Y=Y_{0} \oplus Y_{1}$ by $Y_{0}$ and its orthogonal complement $Y_{1}$ in $Y$, where $Y_{0}$ is the intersection of $Y$ and the kernel of $A^{2}$. We put $d=\operatorname{dim} Y$ and $k=\operatorname{dim} Y_{0}$. (Remark. The ascent of $A$ at zero is at most two because $H$ is non-negative.) Further we use the notations $H_{+}$ $=\left.H\right|_{Y}, H_{+0}=\left.H\right|_{Y_{0}}$ and $H_{+1}=\left.H\right|_{Y_{1}}$ for the restriction of $H$ on $Y, Y_{0}$ and $Y_{1}$. (Remark. In general $Y_{0}$ and $Y_{1}$ are not invariant subspaces of $H$. So the restriction $H_{+j}$ of $H$ on $Y_{j}$ is defined by the matrix on $Y_{j}$ satisfying $\left(H_{+j} f, g\right)$ $=(H f, g)$ for any $f$ and $g$ of $Y_{j}$, that is, $H_{+j}=P\left(Y_{j}\right) H$ by using the orthogonal projection $P\left(Y_{j}\right)$ onto $Y_{j}$.)

Let $\lambda_{j}$ be positive eigenvalues of $A$. They are simple and the number of them is $(d-k) / 2$. $\quad-\lambda_{j}$ are also eigenvalues of $A$ and total eigenvalues consist of zero and them according to non-negativity of $H$. The adjoint matrix $A^{*}$ is given by $i H J$ and has same eigenvalues 0 and $\lambda_{j}$. Let $a_{j}$ be an eigenvector of $A^{*}$ corresponding to $\lambda_{j}$. $\left\{a_{j}\right\}$ are linearly independent of each other by taking as $a_{j}$ and $a_{k}$ are orthogonal if $\lambda_{j}=\lambda_{k}$. And also $\underline{a}_{j}=a_{j}^{\text {conj }}$ is an eigenvector corresponding to $-\lambda_{j}$. If $a$ and $b$ are two eigenvectors of $A^{*}$ corresponding to 
eigenvalues $\lambda$ and $\mu,(\lambda+\mu)\langle J a, b\rangle=0$. And $i \lambda\langle J a, \underline{a}\rangle=\langle H J a, J \underline{a}\rangle\rangle 0$ if $\lambda \neq 0$ because $J a$ does not belong to $N$ if $\lambda \neq 0$. This implies that $\langle J a, \underline{a}\rangle \neq 0$. Therefore we can take $a_{j}$ as $i\left\langle J a_{j}, \underline{a}_{j}\right\rangle=1$ and $\left\langle J a_{j}, a_{k}\right\rangle=0$ because $\lambda_{j}+\lambda_{k} \neq 0$. (Remark. $i\left\langle J a_{j}, \underline{a}_{j}\right\rangle$ is real positive from the first.) Since $\lambda\langle a, x\rangle=-\langle a, A x\rangle$, $\lambda^{2}\langle a, x\rangle=\left\langle a, A^{2} x\right\rangle$ so that $\lambda^{2}\langle a, x\rangle=0$ if $x$ belongs to $N$ or $Y_{0}$. This means that $a$ is orthogonal to $N \oplus Y_{0}$ if $\lambda \neq 0$. Therefore $a_{j}$ belongs to $Y_{1}$. Considering the dimension, $\left\{a_{j}, \underline{a}_{j}\right\}$ is a base of $Y_{1}$. From the above we get the following proposition.

Proposition A.1.1. 1) Let $\lambda_{j}, j=1, \ldots,(d-k) / 2$, be positive eigenvalues of $A$. There exist $a_{J}$ belonging to $Y_{1}$ such that $i\left\langle J a_{j}, \underline{a}_{k}\right\rangle=\delta_{j k},\left\langle J a_{j}, a_{k}\right\rangle=0$, $\left\{a_{j}, \underline{a}_{j}\right\}$ is a base of $Y_{1}$ and $a_{j}$ is an eigenvector of $A^{*}$ corresponding to $\lambda_{j}$.

2) There exists a real base of $Y_{0}$ such that $\left\langle H_{+0} c_{j}, c_{k}\right\rangle=\left\langle H c_{j}, c_{k}\right\rangle=\delta_{j k}$.

3) If we put $b_{j}=H c_{j}$, which belong to $Y$ and satisfy $\left\langle b_{j}, c_{k}\right\rangle=\delta_{j k}$, then we get

$$
A=\sum_{j} \lambda_{j}\left(i J a_{j} \otimes \underline{a}_{j}+i J \underline{a}_{j} \otimes a_{j}\right)+\sum_{j} i J b_{j} \otimes b_{j} .
$$

Remark. $b_{j}, a_{j}$ and $\underline{a}_{j}$ are linearly independent of each other and they make a base of $Y$. $\left\langle J b_{j}, b_{k}\right\rangle=\left\langle H J H c_{j}, c_{k}\right\rangle=0$ for all $j$ and $k$ because $c_{j}$ belongs to $Y_{0} . \quad \lambda_{j}\left\langle a_{j}, c_{k}\right\rangle=-i\left\langle a_{j}, J H c_{k}\right\rangle$ and $\left\langle a_{j}, J b_{k}\right\rangle=0$ if $a_{j}$ belongs to $Y_{1}$ and $c_{k}$ belongs to $Y_{0}$. Therefore $\left\langle J a_{j}, b_{j}\right\rangle=\left\langle J \underline{a}_{j}, b_{k}\right\rangle=0$.

Proof. $A J a_{j}=\lambda_{j} J a_{j}, A J \underline{a}_{j}=-\lambda_{j} J \underline{a}_{j}, A c_{j}=i J b_{j}$ and $A v=0$ if $v$ belongs to $N$. This means that the left hand side is equal to the right hand side because $X=N \oplus Y$.

q.e.d.

Let us put $M=N \oplus Y_{0}$. This is the generalized eigenspace of $A^{*}$ at zero and simplectic, namely, $J$ is non-singular on $M$. In fact, if $\langle J f, u\rangle=0$ for all $u$ of $M$, then $J f$ belongs to $Y_{1}$. This implies that $f=i J H J g$ with some $g$ of $Y_{1}$ so that $0=A^{2} f=A^{3} J g$. Since the ascent of $A$ at zero is at most two, $A^{2} J g$ must vanish. Therefore $J g$ belongs to $Y_{0}$. Pulting $u=J g$, we get that $\langle J f, J g\rangle$ $=\langle f, g\rangle=0$. On the other hand, since $\langle f, g\rangle=-i\langle H J g, J g\rangle, J g$ belongs to $N$. Thus $J g$ belongs to both $Y_{0}$ and $N$, namely, $J g$ must be zero. So we conclude that $f=0$. Since $M$ is simplectic, there exists $d_{j}, j=1, \ldots,(2 n-d+k) / 2$, such that $i\left\langle J \underline{d}_{j}, d_{k}\right\rangle=\delta_{j k},\left\langle J d_{j}, d_{k}\right\rangle=0$. Therefore $\sum_{j}\left(d_{j} \otimes i J \underline{d}_{j}-\underline{d}_{j} \otimes i J d_{j}\right)$ is the identity on $M$. Using these vectors $\left\{a_{j}, b_{j}, d_{j}\right\}$, we can represent functions of $A$ in the following way.

Proposition A.1.2. Let $G(\lambda)$ be a continuously differentiable function in 
$\lambda$ of $\mathbf{R}$. Then $G(A)$ has the following canonical form.

(A.1.1) $G(A)=\sum_{j}\left\{G\left(\lambda_{j}\right) i J a_{j} \otimes \underline{a}_{j}-G\left(-\lambda_{j}\right) i J \underline{a}_{j} \otimes a_{j}\right\}$

$$
+G^{\prime}(0) \sum_{j} i J b_{j} \otimes b_{j}+G(0) \sum_{j}\left(d_{j} \otimes i J \underline{d}_{j}-\underline{d}_{j} \otimes i J d_{j}\right) .
$$

Especially if $G(\lambda)$ is an odd function in $\lambda$, we get

$$
i J G(A)=\sum_{j} G\left(\lambda_{j}\right)\left\{a_{j} \otimes \underline{a}_{j}+\underline{a}_{j} \otimes a_{j}\right\}+G^{\prime}(0) \sum_{j} b_{j} \otimes b_{j} .
$$

Since $a_{j}, \underline{a}_{j}$ and $b_{j}$ are linearly independent on $Y$, there exist $e_{j}$ such that $\left\langle a_{j}, e_{k}\right\rangle=\delta_{j k},\left\langle a_{j}, \underline{e}_{k}\right\rangle=0$ and $\left\langle b_{j}, e_{k}\right\rangle=0$. Then, since $\left\{e_{j}, \underline{e}_{j}, c_{j}\right\}$ is also a base of $Y$, we may induce a coordinate on $Y$ such that $f=x e+y \underline{e}+z c$ for $f$ of $Y$. Therefore we conclude the following.

Proposition A.1.3. If $G$ is an odd function, we get

$$
\langle i J G(A) f, \underline{f}\rangle=\sum_{j} G\left(\lambda_{j}\right)\left(\left|x_{j}\right|^{2}+\left|y_{j}\right|^{2}\right)+G^{\prime}(0)|z|^{2} .
$$

Especially if $G(A)=A$,

$$
\left\langle H f, f_{-}\right\rangle=\sum_{j} \lambda_{j}\left(\left|x_{j}\right|^{2}+\left|y_{j}\right|^{2}\right)+|z|^{2} .
$$

Moreover if $G$ is a real function, then $\langle i J G(A) f, \underline{f}\rangle$ is real.

Let $\left\{f_{j}\right\}$ be another base of $Y$. We denote the coordinate $u_{j}$ of $f$ by it, that is, $f=\sum_{j} u_{j} f_{j}=x e+y \underline{e}+z c$. And we denote the mapping on $\mathbf{C}^{d}$ from $\left(u_{j}\right)$ to $(x, y, z)$ by $\Phi$ and the matrix representation of $H_{+}$with respect to $\left(u_{j}\right)$ variable by also $H_{+}$. Then we get the followings using the above propositions.

\section{Proposition A.1.4.}

$$
H_{+}=\Phi^{*} \Lambda \Phi,
$$

where $\Lambda$ is a diagonal matrix, diagonal elements of which consist of some 1 and two $\lambda_{j}, j=1, \ldots,(d-k) / 2$.

$$
\operatorname{det} H_{+}=\prod_{j=1}^{(d-k) / 2} \lambda_{j}^{2}|\operatorname{det} \Phi|^{2} .
$$

In general putting iJG(A) $\left.\right|_{Y}=G_{+}(G$ is odd $)$, we get

$$
\operatorname{det} G_{+}=\prod_{j=1}^{(d-k) / 2} G\left(\lambda_{j}\right)^{2}|\operatorname{det} \Phi|^{2} .
$$

(*) Proof of Proposition 1.2. Let us consider the case that $H \geqq \delta>0$. $(x, y)_{H}=(H x, y)$ defines another inner product on $X$ and satisfies that $\delta\|x\|^{2}$ $\leqq\|x\|_{H}^{2} \leqq M\|x\|^{2}$, where $M=\|H\|=\sup _{x \neq 0}(H x, x) /\|x\|^{2}$ and \|\|$_{H}$ is the norm with respect to $(,)_{H} \cdot A=i J H$ is a selfadjoint operator with respect to $(,)_{H}$ and $\|A\|_{H} \leqq M$. This implies the following. 
Lemma A.1.1. Let us put $R_{\lambda}=(\lambda-A)^{-1}$ for $\operatorname{Im} \lambda \neq 0$. Then $\left\|R_{\lambda}\right\|_{H} \leqq$ $|\operatorname{Im} \lambda|^{-1}$.

Let us consider the resolvent equation.

$$
\begin{aligned}
& \left\{I-\varepsilon(\lambda-i J H)^{-1} i J\right\}\{\lambda-i J(H+\varepsilon I)\}^{-1} \\
= & \{\lambda-i J(H+\varepsilon I)\}^{-1}\left\{I-\varepsilon i J(\lambda-i J H)^{-1}\right\} \\
= & (\lambda-i J H)^{-1} .
\end{aligned}
$$

This implies that there exists $\{\lambda-i J(H+\varepsilon I)\}^{-1}$ if there exist $(\lambda-i J H)^{-1}$, $\left\{I-\varepsilon(\lambda-i J H)^{-1} i J\right\}^{-1}$ and $\left\{I-\varepsilon i J(\lambda-i J H)^{-1}\right\}^{-1}$. Let us put $K_{1}=\varepsilon(\lambda-i J H)^{-1} i J$ and $K_{2}=\varepsilon i J(\lambda-i J H)^{-1}$.

Lemma A.1.2. $\left\|K_{j}\right\|_{H}^{2} \leqq \varepsilon^{2}(M / \delta)|\operatorname{Im} \lambda|^{-2},(j=1,2)$.

Proof. Let us put $K_{1} f=u_{1}$ and $K_{2} f=u_{2}$, that is, $\varepsilon i J f=(\lambda-i J H) u_{1}$ and $\varepsilon f=(\lambda-i J H) i J u_{2} . \quad$ By Lemma A.1.1, $\left\|u_{1}\right\|_{H}^{2} \leqq \varepsilon^{2}|\operatorname{Im} \lambda|^{-2}\|i J f\|_{H}^{2}$ and $\left\|i J u_{2}\right\|_{H}^{2}$ $\leqq \varepsilon^{2}|\operatorname{Im} \lambda|^{-2}\|f\|_{H}^{2}$. Here we know that $\|i J f\|_{H}^{2} \leqq M\|f\|^{2} \leqq(M / \delta)\|f\|_{H}^{2}$ and $\left\|u_{2}\right\|_{H}^{2} \leqq M\left\|i J u_{2}\right\|^{2} \leqq(M / \delta)\left\|i J u_{2}\right\|_{H}^{2}$. This implies that $\left\|i J u_{1}\right\|_{H}^{2} \leqq \varepsilon^{2} M \delta^{-1}|\operatorname{Im} \lambda|^{-2}$ $\times\|f\|_{H}^{2}$ and $\left\|u_{2}\right\|_{H}^{2} \leqq \varepsilon^{2} M \delta^{-1}\left|\operatorname{Im} \lambda_{1}\right|^{-2}\|f\|_{H}^{2}$. We get the conclusions. q.e.d.

Now let us assume that $G$ is a real symmetric matrix, and let us put $H$ $=G+\left(\delta+\delta_{0}\right) I,(\delta>0)$, where $\delta_{0}=-\inf _{f \neq 0}(G f, f) /\|f\|^{2}$. And also we put $L=\sup _{f \neq 0}(G f, f) /\|f\|^{2}$ and $M=L+\delta+\delta_{0}$.

Lemma A.1.3. $M\|f\|^{2} \geqq(H f, f) \geqq \delta\|f\|^{2}$.

Proposition A.1.5. (Proposition 1.2.)

1) If $2^{-1}\left\{L+\left(L^{2}+3|\operatorname{Im} \lambda|^{2}\right)^{1 / 2}\right\}^{-1}|\operatorname{Im} \lambda|^{2} \geqq \delta_{0}$ and $\operatorname{Im} \lambda \neq 0$, then there exists $(\lambda-i J G)^{-1}$.

2) If $2^{-1}\left\{L+\left(L^{2}+3 k^{2}|\operatorname{Im} \lambda|^{2}\right)^{1 / 2}\right\}^{-1} k^{2}|\operatorname{Im} \lambda|^{2} \geqq \delta_{0}$ for some $k$ such that $0<k<1$, we get

$$
\begin{aligned}
& \left\|(\lambda-i J G)^{-1}\right\| \\
& \quad \leqq\left(4 L^{2}+5 k^{2}|\operatorname{Im} \lambda|^{2}\right)^{1 / 2}\{k(1-k)\}^{-1}|\operatorname{Im} \lambda|^{-2}, \text { when } L \geqq 0,
\end{aligned}
$$

or

$$
\leqq 2^{1 / 2}(1-k)^{-1}|\operatorname{Im} \lambda|^{-1}, \quad \text { when } L \leqq 0 .
$$

Proof. Since $H=G+\left(\delta+\delta_{0}\right) I \geqq \delta$, there exists $\{\lambda-i J(H+\varepsilon I)\}^{-1}$ by Lemma A.1.2 and Lemma A.1.3 if $\varepsilon^{2} M \delta^{-1}|\operatorname{Im} \lambda|^{-2}<k^{2}$ and $0<k^{2} \leqq 1$. Here we put $\varepsilon=-\delta-\delta_{0}$. Then $H+\varepsilon I=G$. Therefore if $\left(\delta+\delta_{0}\right)^{2}\left(L+\delta+\delta_{0}\right) \delta^{-1}$ $\times|\operatorname{Im} \lambda|^{-2}<k^{2} \leqq 1$, there exists $(\lambda-i J G)^{-1}$. The domain of $\lambda$ such that there 
exists $\delta(>0)$ which satisfies $\left(\delta+\delta_{0}\right)^{2}\left(L+\delta+\delta_{0}\right) \delta^{-1}|\operatorname{Im} \lambda|^{-2}<k^{2}$ is obtained by putting $\beta=L, \alpha=|\operatorname{Im} \lambda|^{2} k^{2}$ and $k=1$ at the following Lemma A.1.4. If $2^{-1}\left\{L+\left(L^{2}+3|\operatorname{Im} \lambda|^{2}\right)^{1 / 2}\right\}^{-1}|\operatorname{Im} \lambda|^{2} \geqq \delta_{0}$ and $|\operatorname{Im} \lambda| \neq 0$, there exists $\delta>0$, that is, there exists $(\lambda-i J G)^{-1}$. Noting Lemma A.1.2 and Lemma A.1.4, we can put $\delta=-\delta_{0}+\alpha\left\{L+\left(L^{2}+3 \alpha\right)^{1 / 2}\right\}^{-1}$ and $\alpha=k^{2}|\operatorname{Im} \lambda|^{2}$. Then $\left\|K_{j}\right\|_{H}<k$. And also $\left\|(\lambda-i J G)^{-1}\right\|_{H} \leqq\left\|(\lambda-i J H)^{-1}\right\|_{H}\left\|\left(I-K_{j}\right)^{-1}\right\|_{H}$. On the other hand $\left\|(\lambda-i J H)^{-1}\right\|_{H} \leqq|\operatorname{Im} \lambda|^{-1}$ by Lemma A.1.1. Therefore we get $\left\|(\lambda-i J G)^{-1}\right\|$ $\leqq(1-k)^{-1}|\operatorname{Im} \lambda|^{-1}$, because $\left\|\left(I-K_{j}\right)^{-1}\right\|_{H} \leqq(1-k)^{-1}$. Denoting $(\lambda-i J G)^{-1}$ by $C,\|C f\|^{2} \leqq \delta^{-1}\|C f\|_{H}^{2} \leqq \delta^{-1}(1-k)^{-2}|\operatorname{Im} \lambda|^{-2}\|f\|_{H}^{2} \leqq M \delta^{-1}(1-k)^{-2}|\operatorname{Im} \lambda|^{-2}$ $\times\|f\|^{2}$. Thus $\|C\| \leqq M^{1 / 2} \delta^{-1 / 2}(1-k)^{-1}|\operatorname{Im} \lambda|^{-1}=\left(L+\delta+\delta_{0}\right)^{1 / 2} \delta^{-1 / 2}(1-k)^{-1}$ $\times|\operatorname{Im} \lambda|^{-1}$. Putting $\gamma=\delta+\delta_{0}=\alpha\left\{L+\left(L^{2}+3 \alpha\right)^{1 / 2}\right\}^{-1}$, we get $\delta \geqq 2^{-1} \gamma$ because $\delta_{0}$ $\leqq 2^{-1}\left\{L+\left(L^{2}+3 \alpha\right)^{1 / 2}\right\}^{-1} \alpha$ and $\alpha=k^{2}|\operatorname{Im} \lambda|^{2}$. If $L>0$, then $\left(L+\delta+\delta_{0}\right) \delta^{-1}$ $\leqq 2(L+\gamma) \gamma^{-1}=2\left(1+L\left\{L+\left(L^{2}+3 \alpha\right)^{1 / 2}\right\} \alpha^{-1}\right) \leqq 2+2\left\{L^{2}+2^{-1}\left(2 L^{2}+3 \alpha\right)\right\} \alpha^{-1}=\left(4 L^{2}\right.$ $+5 \alpha) \alpha^{-1}$. Therefore $\|C\| \leqq\left(4 L^{2}+5 \alpha\right)^{1 / 2} \alpha^{-1 / 2}(1-k)^{-1}|\operatorname{Im} \lambda|^{-1} \leqq\left(4 L^{2}+5 k^{2}\right.$ $\left.\times|\operatorname{Im} \lambda|^{2}\right)^{1 / 2}\{k(1-k)\}^{-1}|\operatorname{Im} \lambda|^{-2}$. If $L \leqq 0$, then $\left(L+\delta+\delta_{0}\right) \delta^{-1} \leqq 2(L+\gamma) \gamma^{-1} \leqq 2$. Therefore $\|C\| \leqq 2^{1 / 2}(1-k)^{-1}|\operatorname{Im} \lambda|^{-1}$.

q.e.d.

Lemma A.1.4. Let us consider a function $f(x)=\left(x+\delta_{0}\right)^{3}+\beta\left(x+\delta_{0}\right)^{2}-\alpha x$, where $\delta_{0} \geqq 0$ and $\alpha>0$. If $2^{-1}\left(\beta+\left(\beta^{2}+3 \alpha\right)^{1 / 2}\right)^{-1} \alpha \geqq \delta_{0}$, then there exists $\delta$ such that $\delta>0$ and $f(\delta)<0$. In fact it is enough to put $\delta=-\delta_{0}+\alpha\left\{\beta+\left(\beta^{2}\right.\right.$ $\left.+3 \alpha)^{1 / 2}\right\}^{-1}$. Then $\delta$ satisfies $\delta \geqq 2^{-1}\left\{\beta+\left(\beta^{2}+2 \alpha\right)^{1 / 2}\right\}^{-1} \alpha$.

Proof. If there exists $\delta>0$ such that $f^{\prime}(\delta)=0$, it is what we need. We put $F(y)=y^{3}+\beta y^{2}-\alpha y+\alpha \delta_{0}$, that is, $F\left(y+\delta_{0}\right)=f(y) . \quad F(0)=\alpha \delta_{0} \geqq 0$ and $F^{\prime}(y)$ $=3 y^{2}+2 \beta y-\alpha$. A solution $\gamma>0$ of $F^{\prime}(\gamma)=0$ is given by $\gamma=\alpha\left\{\beta+\left(\beta^{2}\right.\right.$ $\left.+3 \alpha)^{1 / 2}\right\}^{-1}$. Since $\gamma>\delta_{0}$ by the assumption, $\delta=\gamma-\delta_{0}$ is positive and $F(\gamma)$ $=f(\delta)$.

$$
F(\gamma)=\alpha\left\{\delta_{0}-\alpha\left(\beta+\left(\beta^{2}+3 \alpha\right)^{1 / 2}\right)^{-1}\left[\left(4 \alpha+\beta^{2}\right)\left(6 \alpha+\beta^{2}+\beta\left(\beta^{2}+3 \alpha\right)^{1 / 2}\right)^{-1}\right]\right\} .
$$

Here $\quad\left(4 \alpha+\beta^{2}\right)\left(6 \alpha+\beta^{2}+\beta\left(\beta^{2}+3 \alpha\right)^{1 / 2}\right)^{-1} \geqq\left(8 \alpha+2 \beta^{2}\right)\left(15 \alpha+4 \beta^{2}\right)^{-1}>2^{-1}(\alpha>0)$. Therefore we get $F(\gamma)<\alpha\left(\delta_{0}-2^{-1}\left(\beta+\left(\beta^{2}+3 \alpha\right)^{1 / 2}\right)^{-1} \alpha\right) \leqq 0$.

q.e.d.

\section{§ A.2. Pseudodifferential Operators}

We mention properties of pseudodifferential operators, which are used in the previous sections, and we give proofs for some of them.

At first we give the formula of transformation between ordinary symbols (A.2.1) and Weyl symbols (A.2.2) because we have used Weyl symbols through 
this paper.

$$
\begin{aligned}
& p^{o}(x, D) u=(2 \pi)^{-n} \int e^{i(x-y) \xi} p(x, \xi) u(y) d y d \xi . \\
& p^{w}(x, D) u=(2 \pi)^{-n} \int e^{i(x-y) \xi} p(x / 2+y / 2, \xi) u(y) d y d \xi .
\end{aligned}
$$

Remark. We do not distinguish between oscillatory integrals and ordinary integrals in notations.

Theorem A.2.1 [Transformation formula between ordinary symbols and Weyl symbols]. If a Weyl symbol $p(x, \xi)$ and an ordinary symbol $q(x, \xi)$ give a same pseudodifferential operator, that is, $p^{w}(x, D)=q^{o}(x, D)$, then they are transformed to each other by the following relations (A.2.3-4).

$$
\begin{aligned}
& q(x, \xi)=(2 \pi)^{-n} \int e^{-i z \zeta} p(x+z / 2, \xi+\zeta) d z d \zeta . \\
& p(x, \xi)=(2 \pi)^{-n} \int e^{i z \zeta} q(x+z / 2, \check{\zeta}+\zeta) d z d \zeta .
\end{aligned}
$$

Remark. In the rest we use only Weyl symbol so that we omit $w$ of $p^{w}(x, D)$ except for Lemma A.2.1 and the proof of Theorem A.2.1.

Theorem A.2.2 [Change of coordinate]. Let us put $f(x)=p(x, D) u(x)$ and $x=\phi(y)$ a diffeomorphism on $\mathbb{R}^{n}$. We assume that $p(x, \xi)$ vanishes out side of a bounded set in $x$. Then by' (A.2.5) we get $g(y, \eta)$ such that $\left(\phi^{*} f\right)$ $(y)=q\left(y, D_{y}\right)\left(\phi^{*} u\right)(y)$.

$$
\text { (A.2.5) } \begin{aligned}
q(y, \eta)= & (2 \pi)^{-n} \int e^{i z \zeta} p\left(\phi(y+z / 2) / 2+\phi(y-z / 2) / 2,{ }^{t} \Psi(z)^{-1}(\zeta+\eta)\right) \\
& \times|\Psi(z)|^{-1}|(\partial / \partial y) \phi(y-z / 2)| d z d \zeta
\end{aligned}
$$

where $\Psi(z)=\Psi(y, z)$ is a matrix valued infinitely differentiable function such that $\phi(y+z / 2)-\phi(y-z / 2)=\Psi(y, z) z$ and that $\operatorname{det} \Psi(z)$ does not vanish on the whole space.

Remark. If $(\partial \phi / \partial y), \Psi, \Psi^{-1}$ and their derivatives are uniformly bounded, we can remove the condition with respect to the support of $p$.

Remark. It is natural for pseudodifferential operators on manifolds to be defined as operators from $\varepsilon$-densities to $(1-\varepsilon)$-densities, $(0 \leqq \varepsilon \leqq 1)$. One of reasons is that principal symbols $p_{m}$ and subprincipal symbols $p_{m-1}$ are well 
defined on cotangent manifolds as homogeneous parts with top order and with next order of symbols $p$ of local expressions when pseudodifferential operators are classical types. This result is easily deduced from Theorem A.2.2. In this paper we consider them as operators from functions to densities. In order to guarantee iterations we fix a positive density $d M$ on a manifold $M$ to identify spaces of functions and densities. With respect to a local chart $(x, U)$ a local expression of a pseudodifferential operator $P$ is given by (A.2.6) with a Weyl symbol $p$ if the density $d M$ is flat with respect to $x$, that is, $d M=d x$ on $U$.

$$
(P u)(x)=(p(x, D) u)(x) \text { on } U \text { for } u \text { of } C_{0}^{+\infty}(U),
$$

where the right hand side is defined as pseudodifferential operators with Weyl symbols on $\mathbf{R}^{n}$.

Remark. We denote the symbol of multi-product of pseudodifferential operators $p_{1}(x, D) p_{2}(x, D) \cdots p_{v}(x, D)$ by $\left(p_{1} \circ p_{2} \circ \cdots \circ p_{v}\right)(x, \xi)$.

Theorem A.2.3 [Formula of multi-product Weyl product]. The symbol of multi-product is given by

$$
\begin{aligned}
& \left(p_{1} \circ \cdots \circ p_{v}\right)(x, \xi) \\
& =2^{n} \int \exp \left\{i \sum_{j=1}^{v} \eta_{j}\left(y_{j}-y_{j+1}\right)\right\} \\
& \quad \times \prod_{j=1}^{v} p_{j}\left(x+y_{j} / 2+y_{j+1} / 2, \xi+\eta_{j}\right) d y_{1} \cdots d y_{v} d \eta_{1} \cdots d \eta_{v} . \\
& =2^{n} \int \exp \left\{i \sum_{j=1}^{v} y_{j}\left(\eta_{j+1}-\eta_{j}\right)\right\} \\
& \quad \times \prod_{j=1}^{v} p_{j}\left(x+y_{j}, \xi+\eta_{j} / 2+\eta_{j+1} / 2\right) d y_{1} \cdots d y_{v} d \eta_{1} \cdots d \eta_{v},
\end{aligned}
$$

where $d \eta_{j}=(2 \pi)^{-n} d \eta_{j}, \eta_{n+1}=-\eta_{1}$ and $y_{n+1}=-y_{1}$.

Remark. For $p$ of $S_{\rho, \delta}^{m}$ we introduce a seminorm $|p|_{l}^{(m)}$ by

$$
|p|_{l}^{(m)}=\sup _{|\alpha|+|\beta| \leqq l}\left[\max _{(x, \xi)}\left\{p_{(\beta)}^{(\alpha)}(x, \xi)\langle\xi\rangle^{-m+\rho|\alpha|-\delta|\beta|}\right\}\right] \text {. }
$$

Theorem A.2.4 [Estimate of multi-product]. Let $p_{j}$ be pseudodifferential operators belonging to $S_{\rho, \delta}^{m(j)}(j=1, \ldots, v)$ and $p=p_{1} \circ p_{2} \circ \cdots \circ p_{v}$. If $\delta<1$ and

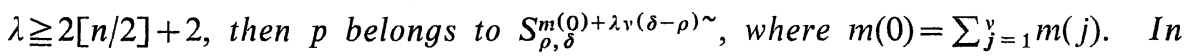
detail for any $l$ there exist $l_{0}$ and $C$, which is independent of $v$, such that the estimate (A.2.8) is satisfied, where $l_{0}$ and $C$ may depend on $l, \lambda$ and $\sum_{j=1}^{v}\left|m(j)+\lambda(\delta-\rho)^{\sim}\right|$.

$$
|p|_{l}^{(m(0)+\lambda v(\delta-\rho) \sim)} \leqq C^{v} \prod_{j=1}^{v}\left|p_{j}\right|_{i+l_{0}}^{(m(j))} .
$$

Remark. 1) $(\delta-\rho)^{\sim}=\max \{\delta-\rho, 0\}$. 2) If $\delta \leqq \rho$, this theorem is one used in Chapter 2. 
Theorem A.2.5 [Expansion formula]. Let $p_{j}$ belong to $S_{\rho(j), \delta(j)}^{m(j)}$ $(j=1, \ldots, v)$, where $\delta(j)<1$ and $\rho(j)>\delta(k)$ if $j \neq k$. For any integer $N \geqq 0$, there exists $q_{N}$ belonging to $S_{\rho, \delta}^{m(0)-\varepsilon N}$ such that

(A.2.9) $\left(p_{1} \circ \cdots \circ p_{v}\right)(x, \xi)$

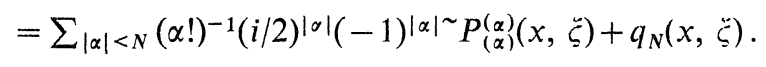

Here $m(0)=\sum_{j=1}^{v} m(j), \rho=\min _{j} \rho(j), \delta=\max _{j} \delta(j), \varepsilon=\min _{j \neq k} \rho(j)-\delta(k), \quad P$ $=\left(p_{1}, \ldots, p_{v}\right), \alpha=\left(\alpha_{j}^{k}\right)$ are systems of multi-indices $\alpha_{j}^{k}(j, k=1, \ldots, v), \alpha_{j}^{k}$ are multi-indices with the same width $n$ (number of indices) such that $\alpha_{j}^{j}$ consist of only zero,

$$
\begin{gathered}
h(\alpha, j)=\sum_{k=1}^{v} \alpha_{j}^{k}, \quad v(\alpha, k)=\sum_{j=1}^{v} \alpha_{j}^{k}, \quad \alpha !=\prod_{j, k=1}^{v}\left(\alpha_{j}^{k}\right), \\
|\alpha|=\sum_{j, k=1}^{v}\left|\alpha_{j}^{k}\right|, \quad|\alpha|^{\sim}=\sum_{j<k}\left|\alpha_{j}^{k}\right| \quad \text { and } \quad P_{(\beta)}^{(\alpha)}=\prod_{j=1}^{v} p_{j(h(\beta, j))}^{(v), j) !} \\
\left(p_{(h)}^{(v)}=\partial_{\xi}^{v} \partial_{x}^{h} p\right) .
\end{gathered}
$$

Theorem A.2.6 [Expansion formula in case of two pseudodifferential operators]. Let us put $\sigma_{k}\left(p_{1}, p_{2}\right)=\sum_{|\alpha+\beta|=k}(-1)^{|\beta|} C_{\alpha \beta}^{k} p_{1(\beta)}^{(\alpha)} p_{2(\alpha)}^{(\beta)}$, where $\alpha$ and $\beta$ are multi-indices, and $C_{\alpha \beta}^{k}=k ! / \alpha ! \beta !$. Then we get the expansion (A.2.10) with $q_{N}$ belonging to $S_{\rho, \delta}^{m(0)-\varepsilon N}$, where $p_{j}$ belongs to $S_{\rho(j), \delta(j)}^{n(j)}, m(0)$ $=m(1)+m(2), \rho=\min \{\rho(1), \rho(2)\}, \delta=\max \{\delta(1), \delta(2)\}$ and $\varepsilon=\min \{\rho(1)-\delta(2)$, $\rho(2)-\delta(1)\}>0$.

$$
p_{1} \circ p_{2}=\sum_{k=0}^{N-1}(2 i)^{-k}(k !)^{-1} \sigma_{k}\left(p_{1}, p_{2}\right)+q_{N} .
$$

Moreover there exist constants $l_{0}$ and $C$ for any $l$ such that

$$
\begin{aligned}
\left|q_{N}\right|_{l}^{(m(0)-\varepsilon N)} \leqq & C \sum_{|\alpha+\beta|=N}\left|p_{1(\beta)}^{(\alpha)}\right|_{l+l_{0}}^{(m(1)+\delta(1)|\beta|-\rho(1)|\alpha|)} \\
& \times\left|p_{2(\alpha)}^{(\beta)}\right|_{l+l_{0}}^{(m(2)+\delta(2)|\alpha|-\rho(2)|\beta|)}
\end{aligned}
$$

where the seminorms are ones of $S_{\rho, \delta}$.

Remarks on $\sigma_{k}$. Let $X$ be $\mathbf{C}^{2 n}$. We define a nondegenerate bilinear form $\sigma_{1}$ on $X^{*}$ by $\sigma_{1}((x, \xi),(y, \eta))=\langle\xi, y\rangle-\langle x, \eta\rangle$. It is extended on the covariant tensor product $T_{k}(X)$ of $X$ by putting it as $\sigma_{k}(u, v)=\prod_{j=1}^{k} \sigma_{1}\left(u_{j}, v_{j}\right)$ for monomials $u=u_{1} \otimes \cdots \otimes u_{k}$ and $v=v_{1} \otimes \cdots \otimes v_{k}$. The restriction of $\sigma_{k}$ on the symmetric tensor $S_{k}(X)$ gives the natural extension of $\sigma_{1}$. Then they satisfy for $u=\xi^{\alpha} x^{\beta}$ and $v=\xi^{\gamma} x^{\delta}$

$$
\sigma_{k}(u, v)=(-1)^{|\beta|} C_{\alpha \beta}^{k}, \quad \text { if } \alpha=\delta \text { and } \beta=\gamma,
$$

or

$$
\sigma_{k}(u, v)=0, \quad \text { otherwise }
$$




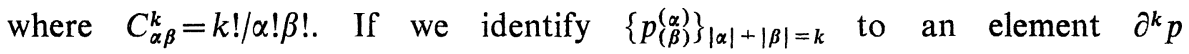
$=\sum_{|\alpha|+|\beta|=k} p_{(\beta)}^{(\alpha)} \xi^{\alpha} x^{\beta}$ of $S_{k}(X)$, then

$$
\sigma_{k}\left(p_{1}, p_{2}\right)=\sigma_{k}\left(\partial^{k} p_{1}, \partial^{k} p_{2}\right) .
$$

Therefore we can deduce the following properties.

Theorem A.2.7. We assume that $p, q, \phi, f$ and $g$ are scalar functions. We identify $X$ and $X^{*}$ by the canonical bilinear form $\left\langle z, z^{\prime}\right\rangle=\sum_{j=1}^{2 n} z_{j} z^{\prime}{ }_{j}$ for $z=\left(z_{j}\right)$ and $z^{\prime}=\left(z^{\prime}{ }_{j}\right)$.

1) $\sigma_{k}(p, q)=(-1)^{k} \sigma_{k}(q, p)$.

2) $\sigma_{1}(p, q)=\langle J \partial p, \partial q\rangle$.

3) $\sigma_{2}(p, q)=-\operatorname{Tr}\left(J H_{p} J H_{q}\right)$.

4) $\sigma_{1}(p, \exp \phi)=\sigma_{1}(p, \phi) \exp \phi$.

5) $\sigma_{1}(p, f g)=\sigma_{1}(p, f) g+\sigma_{1}(p, g) f$.

6) $\sigma_{2}(p, f g)=\sigma_{2}(p, f) g+\sigma_{2}(p, g) f+2\left\langle J \partial f, H_{p} J \partial g\right\rangle$.

7) $\sigma_{2}(p, \exp \phi)=\sigma_{2}(p, \phi) \exp \phi+\left\langle J \partial \phi, H_{p} J \partial \phi\right\rangle \exp \phi$.

Here $H_{\psi}=\partial^{2} \psi$ and $\sigma_{1}(u, v)=\langle J u, v\rangle$.

Proof. 1) and 2) are clear from the definition, where $J$ is a linear mapping such that $J x=-\xi$ and $J \xi=x$. 3) For two monomials $u=u_{j} \otimes u_{k}$ and $v=$ $v_{j} \otimes v_{k}$, it means that

$$
\begin{aligned}
\sigma_{2}(u, v) & =\sigma_{1}\left(u_{j}, v_{j}\right) \sigma_{1}\left(u_{k}, v_{k}\right) \\
& =\left\langle J u_{j}, v_{j}\right\rangle\left\langle J u_{k}, v_{k}\right\rangle \\
& =-\operatorname{Tr}\left(J^{t} u \cdot J v\right) .
\end{aligned}
$$

4-7) are proved by noting that $\partial(\exp \phi)=\partial \phi \exp \phi, \partial(f g)=g \partial f+f \partial g, \partial^{2}(f g)$ $=g \partial^{2} f+2 \partial f \partial g+f \partial^{2} g$ and $\partial^{2}(\exp \phi)=\partial^{2} \phi \exp \phi+\partial \phi \partial \phi \exp \phi$.

Lemma A.2.1. Let us define an operator $K$ for a kernel $k(x, y)$ of $\mathscr{S}_{(x, y)}$ by

$$
(K u)(x)=\int k(x, y) u(y) d y .
$$

If we put $k(x, y)=k_{1}^{\prime}((x+y) / 2, x-y)=k_{2}^{\prime}(x, x-y)$ and define $k_{j}^{\sim}(x, \xi)(j=1,2)$ by

$$
k_{j}^{\sim}(x, \xi)=\int e^{-i y \xi} k_{j}^{\prime}(x, y) d y .
$$

Then $K u=k_{1}^{\sim w}(x, D) u=k_{2}^{o}(x, D) u$.

Proof. 


$$
k_{1}^{\prime}((x+y) / 2, x-y)=(2 \pi)^{-n} \int e^{i(x-y) \xi} k_{1}((x+y) / 2, \xi) d \xi
$$

and

$$
k_{2}^{\prime}(x, x-y)=(2 \pi)^{-n} \int e^{i(x-y) \xi} k_{2}(x, \zeta) d \xi .
$$

Substitute them into the definition of $K$.

q.e.d.

Proof of Theorem A.2.1. It suffices to prove it in the case that one of $p$ and $q$ belongs to $\mathscr{S}$ as a function in $(x, \xi)$ because it implies that the other also belongs to $\mathscr{S}$ if they are connected by the relations of the theorem and because $\mathscr{S}$ is dense in $S_{\rho, \delta}$ in a suitable weak sense. If we put $k_{1}^{\sim}=p\left(k_{2}^{\sim}=q\right)$ in Lemma A.2.1, then $q=k_{2}\left(p=k_{1}^{\sim}\right)$. This implies the first (second) equality. q.e.d.

Proof of Theorem A.2.2. Let us put $f=p(x, D) u$. Changing variables as $x=\phi(z)$ and $y=\phi(w)$, we get

$$
\left(\phi^{*} f\right)(z)=\int \exp \{i(\phi(z)-\phi(w)) \xi\} p((\phi(z)+\phi(w)) / 2, \xi) v(w)|(\partial / \partial w) \phi| d w d \xi,
$$

where $v=\phi^{*} u$. By Lemma A.2.1 we find a Weyl symbol $q(y, \eta)$ which attains the same operator as

$$
\begin{aligned}
\phi^{*} f(z) & =\int k(z, w) d w \\
k(z, w) & =\int \exp \{i(\phi(z)-\phi(w)) \xi\} p((\phi(z)+\phi(w)) / 2, \xi)|(\partial / \partial w) \phi|(w) d \xi .
\end{aligned}
$$

It is given by

$$
\begin{aligned}
q(y, \eta)= & \int \exp \{-i \eta z+i(\phi(y+z / 2)-\phi(y-z / 2)) \zeta\} \\
& \times p(\phi(y+z / 2) / 2+\phi(y-z / 2) / 2, \zeta)|(\partial / \partial y) \phi(y-z / 2)| d z d \zeta .
\end{aligned}
$$

By the assumption on $\phi$, there exist $\Psi(y, z)$ satisfying that

$$
\phi(y+z / 2)-\phi(y-z / 2)=\Psi(y, z) z
$$

and that det $\Psi(y, z)$ does not vanish. In fact the existence of $\Psi$ satisfying the equality is shown by Taylor's expansion formula. At a neighborhood of $z=0$, $\Psi$ is nonsingular by the assumption. Otherwise adding a certain matrix vanishing at vector $z$, we can make $\Psi$ be nonsingular because $\phi(y+z / 2)-\phi(y-z / 2)$ also does not vanish there. Therefore changing variables $-\eta+{ }^{t} \Psi(y, z) \zeta$ to $\zeta^{\prime}$, then $\zeta={ }^{t} \Psi^{-1}\left(\zeta^{\prime}+\eta\right)$ and $d \zeta=\left|{ }^{t} \Psi^{-1}\right| d \zeta^{\prime}$. We get the equality of the theorem. 
Proof of Theorem A.2.3. If $p_{j}$ belongs to $\mathscr{S}$, we get easily the first equality by linear transformations of variables from the expression obtained by definition and by using Lemma A.2.1. On the second equality the following property of Weyl symbols works effectively.

q.e.d.

Lemma A.2.2. We denote the Fourier transformation of $f$ by $f^{\wedge}$.

$$
(p(x, D) u)^{\wedge}(\eta)=(2 \pi)^{-n} \int e^{-i z(\eta-\zeta)} p(z,(\eta+\zeta) / 2) u^{\wedge}(\zeta) d \zeta d z .
$$

Proof of Theorem A.2.4. At first we prepare two lemmas.

Lemma A.2.3. Let us consider a function

$$
G(\xi, \eta)=1+|\xi-\eta|\{\langle\xi\rangle+|\xi-\eta|\}^{-\delta}
$$

where $0 \leqq \delta<1$.

1) $G(\eta, \xi) \leqq 2 G(\xi, \eta)$.

2) $G(\xi, \zeta) \leqq 2 G(\xi, \eta)$ if $|\xi-\zeta| \leqq 2|\xi-\eta|$.

3) $G(\xi, \eta)^{-N} G(\eta, \zeta)^{-N} \leqq 8^{N} G(\xi, \zeta)^{-N}\left\{G(\zeta, \eta)^{-N}+G(\xi, \eta)^{-N}\right\}$.

4) If $2|\xi-\eta| \leqq\langle\xi\rangle$, then $\langle\eta\rangle \leqq(3 / 2)\langle\xi\rangle$, $\langle\eta\rangle^{-1} \leqq 2\langle\xi\rangle^{-1}$ and $G(\xi, \eta)^{-1} \leqq C\left(1+|\xi-\eta|\langle\xi\rangle^{-\delta}\right)^{-1}$.

5) If $2|\xi-\eta| \geqq\langle\xi\rangle$, then $\langle\eta\rangle \leqq 3|\xi-\eta|,\langle\eta\rangle^{-1} \leqq\langle\xi\rangle^{-1}(1+|\xi-\eta|)$ and $G(\xi, \eta)^{-1} \leqq C\left(1+|\xi-\eta|^{1-\delta}\right)^{-1}$.

6) $\int G(\xi, \eta)^{-N}\langle\eta\rangle^{m} d \eta \leqq C(|m|)\langle\xi\rangle^{m+n \delta}$ if $(1-\delta) N-|m|>n$.

7) Let us put

$$
\Psi_{v}(\xi, \eta)=\prod_{j=1}^{v}\left[G\left(\xi+\eta_{j}, \xi+\eta_{j+1}\right)^{-N}\left\langle\xi+\eta_{j}\right\rangle^{m(j)-n \delta}\right]
$$

where $\eta_{v+1}=-\eta_{1}$ and $\eta=\left(\eta_{1}, \ldots, \eta_{v}\right)$. Then there exists a constant $C$ depending only on $M=\sum_{j=1}^{v}|m(j)|$ such that

$$
\int \Psi_{v}(\xi, \eta) d \eta \leqq C^{v}\langle\xi\rangle^{m} \text { if }(1-\delta) N-M>n,
$$

where $m=\sum_{j=1}^{v} m(j)$.

Proof. 1) Since $\langle\xi\rangle \leqq\langle\eta\rangle+|\xi-\eta|$, we get $(\langle\xi\rangle+|\xi-\eta|) \leqq 2(\langle\eta\rangle+|\xi-\eta|)$. Therefore $G(\xi, \eta) \geqq 2^{-\delta} G(\eta, \xi),(0 \leqq \delta<1)$.

2) When $\delta=0$, it is clear. We assume that $0<\delta<1$. By the assumption, $\langle\xi\rangle|\xi-\eta|^{-1 / \delta} \leqq 2^{1 / \delta}\langle\xi\rangle|\zeta-\zeta|^{-1 / \delta}$ and $|\xi-\eta|^{1-1 / \delta} \leqq 2^{1 / \delta-1}|\xi-\zeta|^{1-1 / \delta}$. Therefore $|\xi-\eta|\{\langle\xi\rangle+|\xi-\eta|\}^{-\delta} \geqq 2^{-1}|\xi-\zeta|\left\{\langle\xi\rangle+2^{-1}|\xi-\zeta|\right\}^{-\delta} \geqq 2^{-1}|\xi-\zeta|\{\langle\xi\rangle+\mid \xi$ $-\zeta \mid\}^{-\delta}$. 
3) $G(\xi, \eta) G(\eta, \zeta) \geqq 2^{-2} G(\xi, \zeta) G(\zeta, \eta)$ if $|\xi-\zeta| \leqq 2|\xi-\eta|$.

$G(\xi, \eta) G(\eta, \zeta) \geqq 2^{-1} G(\xi, \eta) G(\zeta, \eta) \geqq 2^{-2} G(\xi, \eta) G(\zeta, \xi)$

$$
\geqq 2^{-3} G(\xi, \eta) G(\xi, \zeta) \text { if }|\xi-\zeta| \leqq 2|\zeta-\eta| \text {. }
$$

4) and 5) are easy.

6) Change $\langle\eta\rangle^{m}$ to $\langle\xi\rangle^{m}$ and integrate $G(\xi, \eta)^{-N}$ by $\eta$ using 4) and 5).

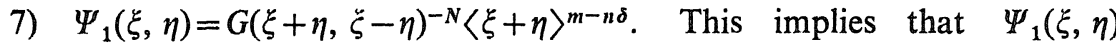
$\leqq C G(\xi, \xi+\eta)^{-N}\langle\xi+\eta\rangle^{m-n \delta}$. Therefore $\int \Psi_{1}(\xi, \eta) d \eta \leqq C\langle\xi\rangle^{m}$ by 6$)$. By the way $\int \Psi_{v}(\xi, \eta) d \eta \leqq C\left(\left|m_{v}\right|\right)\left\{\int \Psi_{v-1}(\xi, \eta) d \eta+\int \Psi_{v-1}^{\prime}(\xi, \eta) d \eta\right\}$ according to 3$)$ and 6), where $m(1)$ in the right hand side is equal to $m(1)+m(v)$ of the left hand side or $m(v-1)$ is equal to $m(v-1)+m(v)$. We can get the conclusion by induction with respect to $v$.

q.e.d.

Next we consider an oscillatory integral

$$
I=\int \exp \left\{i \sum_{j=1}^{v} \eta_{j}\left(y_{j}-y_{j+1}\right)\right\} \prod_{j=1}^{v} q_{j}\left(y_{j}+y_{j+1}, \xi+\eta_{j}\right) d y d \eta,
$$

where $y=\left(y_{1}, \ldots, y_{v}\right), y_{v+1}=-y_{1}$ and $\eta=\left(\eta_{1}, \ldots, \eta_{v}\right)$.

Lemma A.2.4. Let us assume that $q_{j}$ belongs to $S_{\rho, \delta}^{m(j)}$. Then there exists a constant $C(l, r, M)$, which depends only on $l, r$ and $M$, such that

$$
|I| \leqq C(l, r, M)^{v}\langle\xi\rangle^{m+2 l v(\delta-\rho) \sim} \prod_{j=1}^{v}\left|q_{j}\right|_{2 l+4 r}^{(m(j))},
$$

where $(\delta-\rho)^{\sim}=\max \{\delta-\rho, 0\}, m=\sum_{j=1}^{v} m(j), M=\sum_{j=1}^{v}\left|m(j)+2 l(\delta-\rho)^{\sim}\right|$, and $l$ and $r$ are integers such that $l>n / 2$ and $(1-\delta) 2 r-M>n$.

Remark. We may take any real number such that $l \geqq[n / 2]+1$ in $\langle\xi\rangle^{m+2 l v(\delta-\rho) \sim}$, though the constant $C$ necessarily depends on it.

Proof. Let us define $A_{j}, L_{j}, B_{j}$ and $R_{j}(j=1, \ldots, v)$ as follows.

$$
\begin{aligned}
& A_{j}=\left\{1+\Xi_{j}^{2 \delta}\left|y_{j}-y_{j+1}\right|^{2}\right\}^{-1} . \\
& L_{j}=\left\{1+\Xi_{j}^{2 \delta}\left(-\Delta_{\eta_{j}}\right)\right\} . \\
& B_{j}=\left\{1+\left(\Xi_{j}^{\delta}+\Xi_{j+1}^{\delta}\right)^{-2}\left|\eta_{j}-\eta_{j+1}\right|^{2}\right\}^{-1} . \\
& R_{j}=\left\{1+\left(\Xi_{j}^{\delta}+\Xi_{j+1}^{\delta}\right)^{-2}\left(-\Delta_{y_{j+1}}\right)\right\},
\end{aligned}
$$

where $\Xi_{j}=\left\langle\xi+\eta_{j}\right\rangle$ and $\Xi_{v+1}=\Xi_{1}$. They satisfy

$$
\begin{aligned}
& A_{j} L_{j} \exp \left\{i \sum_{j=1}^{v} \eta_{j}\left(y_{j}-y_{j+1}\right)\right\} \\
& \quad=B_{j} R_{j} \exp \left\{i \sum_{j=1}^{v} \eta_{j}\left(y_{j}-y_{j+1}\right)\right\} \\
& \quad=\exp \left\{i \sum_{j=1}^{v} \eta_{j}\left(y_{j}-y_{j+1}\right)\right\} .
\end{aligned}
$$


This implies for any integers $l$ and $r$

$$
\begin{aligned}
I= & \int \exp \left\{i \sum_{j=1}^{v} \eta_{j}\left(y_{j}-y_{j+1}\right)\right\} \\
& \times \prod_{j=1}^{v}\left({ }^{t} L_{j} A_{j}\right)^{l} \prod_{j=1}^{v}\left({ }^{t} R_{j} B_{j}\right)^{r} \\
& \times \prod_{j=1}^{v} q_{j}\left(y_{j}+y_{j+1}, \xi+\eta_{j}\right) d y d \eta .
\end{aligned}
$$

Noting that $q_{j}$ belongs to $S_{\rho, \delta}^{m(j)}$, we have

$$
\begin{aligned}
& \left|\prod_{j=1}^{v}\left({ }^{t} L_{j} A_{j}\right)^{l} \prod_{j=1}^{v}\left({ }^{t} R_{j} B_{j}\right)^{r} \prod_{j=1}^{v} q_{j}\left(y_{j}+y_{j+1}, \xi+\eta_{j}\right)\right| \\
& \leqq C(l, r)^{v} \prod_{j=1}^{v}\left|q_{j}\right|_{2 l+4 r}^{(m(j))} A_{j}^{l} B_{j}^{r}\left\langle\xi+\eta_{j}\right\rangle^{m(j)+2 l(\delta-\rho) \sim ~} .
\end{aligned}
$$

Therefore we get

$$
\begin{aligned}
|I| \leqq & C(l, r)^{v} \prod_{j=1}^{v}\left|q_{j}\right|_{2 l+4 r}^{(m(j))} \\
& \times \int \prod_{j=1}^{v} A_{j}^{l} B_{j}^{r}\left\langle\xi+\eta_{j}\right\rangle^{m(j)+2 l(\delta-\rho) \sim} d y d \eta .
\end{aligned}
$$

On the other hand we know that, if $l>n / 2$,

$$
\int \prod_{j=1}^{v} A_{j}^{l} d y \leqq C^{v} \prod_{j=1}^{v}\left\langle\xi+\eta_{j}\right\rangle^{-n \delta},
$$

and that, if $(1-\delta) 2 r-M>n$,

$$
\begin{aligned}
& \int \prod_{j=1}^{v} B_{j}^{r}\left\langle\xi+\eta_{j}\right\rangle^{m(j)+2 l(\delta-\rho)^{\sim-n \delta}} d \eta \\
& \quad \leqq C^{v} \int \prod_{j=1}^{v} G\left(\xi+\eta_{j}, \xi+\eta_{j+1}\right)^{-2 r}\left\langle\xi+\eta_{j}\right\rangle^{m(j)+2 l(\delta-\rho)^{\sim-n \delta}} d \eta \\
& \quad \leqq C^{v}\langle\xi\rangle^{m+2 l v(\delta-\rho)^{\sim}}
\end{aligned}
$$

where $C$ depends only on $l, r$ and $M$, according to 7) of the previous lemma. Combining these results we get the conclusion.

By Theorem A.2.4,

$$
\begin{aligned}
& \left(p_{1} \circ \cdots \circ p_{v}\right)(x, \xi) \\
& \quad=2^{n} \int \exp \left\{i \sum_{j=1}^{v} \eta_{j}\left(y_{j}-y_{j+1}\right)\right\} \prod_{j=1}^{v} p_{j}\left(x+\left(y_{j}+y_{j+1}\right) / 2, \xi+\eta_{j}\right) d y d \eta
\end{aligned}
$$

Therefore we get

$$
\begin{aligned}
\left(p_{1} \circ \cdots \circ p_{v}\right)_{(\beta)}^{(\alpha)}(x, \xi) & \\
= & \sum_{\alpha=\Sigma \alpha(j) \text { and } \beta=\Sigma \beta(j)}\left\{\alpha ! \beta ! / \prod_{j=1}^{v} \alpha(j) ! \beta(j) !\right\} 2^{n} \\
& \times \int \exp \left\{i \sum_{j=1}^{v} \eta_{j}\left(y_{j}-y_{j+1}\right)\right\} \\
& \times \prod_{j=1}^{v} p_{j(\beta(j))}^{(\alpha(j))}\left(x+\left(y_{j}+y_{j+1}\right) / 2, \xi+\eta_{j}\right) d y d \eta
\end{aligned}
$$


Applying Lemma A.2.4 to

$$
q_{j}(y, \eta)=p_{j(\beta(j))}^{(\alpha(j))}(x+y / 2, \eta),
$$

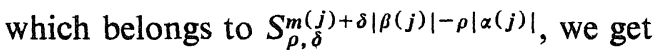

$\left|\left(p_{1} \circ \cdots \circ p_{v}\right)_{(\beta)}^{(\alpha)}(x, \xi)\right|$

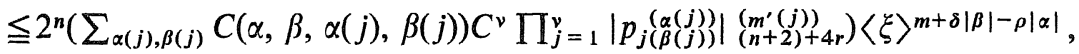

(where $\left.m^{\prime}(j)=m(j)+\delta|\beta(j)|-\rho|\alpha(j)|+2 l(\delta-\rho)^{\sim}\right)$

$\leqq C(\alpha, \beta, M)^{v} \prod_{j=1}^{v}\left|p_{j}\right|_{|\alpha|+|\beta|+(n+2)+4 r}^{(m(j))}\langle\xi\rangle^{m+\delta|\beta|-\rho|\alpha|+(n+2) v(\delta-\rho) \sim ~}$.

Therefore there exists $l_{0}$ for any $l$ such that

$$
\left|p_{1} \circ \cdots \circ p_{v}\right|_{l}^{(m+(n+2) v(\delta-\rho) \sim)} \leqq C(l, M)^{v} \prod_{j=1}^{v}\left|p_{j}\right|_{l+l_{0}}^{(m(j))} .
$$

Proof of Theorems A.2.5-6. Changing variables as $y_{j}-y_{j+1}=z_{j}$ and $\eta_{j}=\zeta_{j}(j=1, \ldots, v)$ at the first part of (A.2.7), we get

$$
\left(p_{1} \circ \cdots \circ p_{v}\right)(x, \xi)=\int \exp \left\{i \sum_{j=1}^{v} \zeta_{j} z_{j}\right\} \prod_{j=1}^{v} p_{j}\left(x+z_{j}^{\prime} / 2, \xi+\zeta_{j}\right) d z d \zeta,
$$

where $z_{j}^{\prime}=\sum_{k=j+1}^{v} z_{k}-\sum_{k=1}^{j-1} z_{k}$. Taking Taylor's expansion with respect to $\zeta$,

$$
\begin{aligned}
& \prod_{j=1}^{v} p_{j}\left(x+z_{j}^{\prime} / 2, \xi+\zeta_{j}\right) \\
& \quad=\sum_{|\alpha|<N}(\alpha !)^{-1} \prod_{j=1}^{v} p_{j}^{(v(\alpha, j))}\left(x+z_{j}^{\prime} / 2, \xi\right) \zeta_{j}^{v(\alpha, j)} \\
& \quad+N \sum_{|\alpha|=N}(\alpha !)^{-1} \zeta^{\alpha} \int_{0}^{1}(1-\theta)^{N-1} \prod_{j=1}^{v} p_{j}^{(v(\alpha, j))}\left(x+z_{j}^{\prime} / 2, \xi+\theta \zeta_{j}\right) d \theta .
\end{aligned}
$$

Noting $\zeta^{\alpha} \exp \{i z \zeta\}=i^{-|\alpha|} \partial_{z}^{\alpha} \exp \{i z \zeta\}$, we take oscillatory integrals of them.

$$
\begin{aligned}
& \left(p_{1} \circ \cdots \circ p_{v}\right)(x, \xi) \\
& =\sum_{|\alpha|<N}(\alpha !)^{-1} i^{|\alpha|} \int \exp \{i z \zeta\} \partial_{z}^{\alpha}\left[\prod_{j=1}^{v} p_{j}^{(v(\alpha, j))}\left(x+z_{j}^{\prime} / 2, \xi\right)\right] d z d \zeta \\
& \quad+\sum_{|\alpha|=N} N(\alpha !)^{-1} i^{|\alpha|} \int \exp \{i z \zeta\} \\
& \quad \times \partial_{z}^{\alpha} \int_{0}^{1}(1-\theta)^{N-1} \prod_{j=1}^{v} p_{j}^{(v(\alpha, j))}\left(x+z_{j}^{\prime} / 2, \xi+\theta \zeta_{j}\right) d \theta d z d \zeta .
\end{aligned}
$$

Execute differentiations in $z$ noting the form of $z_{j}^{\prime}$.

$$
\left(p_{1} \circ \cdots \circ p_{v}\right)(x, \xi)=I_{1}+I_{2},
$$

where

$$
\begin{aligned}
I_{1}= & \sum_{|\alpha|<N}(\alpha !)^{-1}(i / 2)^{|\alpha|}(-1)^{|\alpha| \sim} \\
& \times \int \exp \{i z \zeta\} \prod_{j=1}^{v} p_{j(h(\alpha, j))}^{(v(\alpha, j))}\left(x+z_{j}^{\prime} / 2, \xi\right) d z d \zeta
\end{aligned}
$$


and

$$
\begin{aligned}
I_{2}= & \sum_{|\alpha|=N} N(\alpha !)^{-1}(i / 2)^{|\alpha|}(-1)^{|\alpha|} \int_{0}^{1}(1-\theta)^{N-1} \\
& \times \int \exp \{i z \zeta\} \prod_{j=1}^{v} p_{j(h(\alpha, j))}^{(v(\alpha, j))}\left(x+z_{j}^{\prime} / 2, \xi+\theta \zeta_{j}\right) d z d \zeta d \theta .
\end{aligned}
$$

Noting that it is an oscillatory integral, we get

$$
\begin{aligned}
I_{1} & =\sum_{|\alpha|<N}(\alpha !)^{-1}(i / 2)^{|\alpha|}(-1)^{|\alpha| \sim} \prod_{j=1}^{v} p_{j(h(\alpha, j))}^{(v(\alpha) j)}(x, \xi) \\
& =\sum_{|\alpha|<N}(\alpha !)^{-1}(i / 2)^{|\alpha|}(-1)^{|\alpha| \sim} P_{(\alpha)}^{(\alpha)}(x, \xi) .
\end{aligned}
$$

Change variables $(z, \zeta)$ to $\left(\theta z, \theta^{-1} \zeta\right)$ in $I_{2}$.

$$
\begin{aligned}
I_{2}= & \sum_{|\alpha|=N} N(\alpha !)^{-1}(i / 2)^{|\alpha|}(-1)^{|\alpha| \sim} \int_{0}^{1}(1-\theta)^{N-1} \\
& \times \int \exp \{i z \zeta\} \prod_{j=1}^{v} p_{j(h(\alpha, j))}^{(v(\alpha, j))}\left(x+\theta z_{j}^{\prime} / 2, \xi+\zeta_{j}\right) d z d \zeta d \theta .
\end{aligned}
$$

Return variables $(z, \zeta)$ to $(y, \eta)$.

$$
\begin{aligned}
I_{2}= & \sum_{|\alpha|=N} N(\alpha !)^{-1}(i / 2)^{|\alpha|}(-1)^{|\alpha|} \int_{0}^{1}(1-\theta)^{N-1} \\
& \times 2^{n} \int \exp \left\{i \sum_{j=1}^{v} \eta_{j}\left(y_{j}-y_{j+1}\right)\right\} \\
& \times \prod_{j=1}^{v} p_{j(h(\alpha, j))}^{\left(\begin{array}{c}
(\alpha, j)) \\
(\alpha, j)
\end{array}\right.}\left(x+\theta\left(y_{j}+y_{j+1}\right) / 2, \xi+\eta_{j}\right) d y d \eta d \theta .
\end{aligned}
$$

Applying Lemma A.2.4 as $q_{j}\left(y_{j}, \eta_{j}\right)=p_{j(h(\alpha, j))}^{(v(\alpha, j))}\left(x+\theta y_{j}, \eta_{j}\right), I_{2}(x, \xi)$ belongs to $S_{\rho, \delta}^{m-\varepsilon N+\lambda(\delta-\rho)^{\sim}}, \lambda=2[n / 2]+2$. In order to get the result we consider a sufficiently large $N^{\prime}$ for a given $N$. Then $I_{2}(x, \zeta)$ with respect to $N^{\prime}$ belongs to $S_{\rho, \delta}^{m-\varepsilon N}$ because we may take $N^{\prime}$ such that $\varepsilon N \leqq \varepsilon N^{\prime}+\lambda(\delta-\rho)^{\sim}$. On the other hand,

$$
\sum_{N \leq|\alpha|<N^{\prime}}(\alpha !)^{-1}(i / 2)^{|\alpha|}(-1)^{|\alpha| \sim P_{(\alpha)}^{(\alpha)}(x, \xi)}
$$

also belongs to $S_{\rho, \delta}^{m-\varepsilon N}$. Therefore the remainder term $q_{N}(x, \xi)$ should belong to $S_{\rho, \delta}^{m-\varepsilon N}$. When $v=2$, the estimate (A.2.11) is easily obtained by estimating $q_{N^{\prime}}$ by Lemma A.2.4 and directly $P_{(\alpha)}^{(\alpha)}$ for $\alpha$ such that $N \leqq|\alpha| \leqq N^{\prime}$. $\quad$ q.e.d.

Remark. Refer to L. Hörmander [6] for other informations about Weyl symbols. 


\section{References}

[1] Beals, R., Characterization of pseudodifferential operators and applications, Duke Math. J., 44 (1977), 45-57.

[2] Boutet de Monvel, L., Grisis, A. and Helffer, B., Paramétrixes d'opérateurs pseudodifférentiels à caractéristiques multiples, Astérique, 34-35 (1976), 93-121.

[ 3 ] Calderon, A. P. and Vaillancourt, R., A class of bounded pseudo-differential operators, Proc. Nat. Acad. Sci. USA, 69 (1972), 1185-1187.

[4] Helffer, B., Quelques examples d'opérateurs pseudo-différentiels localement résolubles, Lecture Notes in Math., 660, Springer, 1978, 88-116.

[5] Hörmander, L., A class of hypoelliptic pseudodifferential operators with double characteristics, Math. Ann., 217 (1975), 165-188.

[6] — The Weyl calculus of pseudo-differential operators, Comm. pure appl. Math., 32 (1979), 359-443.

[7] Iwasaki, C., The fundamental solution for pseudo-differential operators of parabolic type, Osaka J. Math., 14 (1977), 569-592.

[8] Iwasaki, C. and Iwasaki, N., Parametrix for a degenerate parabolic equation, Proc. Japan Acad., 55 (1979), 237-240.

[9] --_, Parametrix for a degenerate parabolic equation and its applications, RIMS Kokyuroku, 376, Kyoto Univ., 1980, 88-100 (in Japanese).

[10] Melin, A., Lower bounds for pseudo-differential operators, Ark. Mat., 9 (1971), 117-140.

[11] Melin, A. and Sjöstrand, J., Fourier integral operators with complex-valued phase functions, Lecture Notes in Math., 459, Springer, 1975, 120-233.

[12] Menikoff, A. and Sjöstrand, J., On the eigenvalues of a class of hypoelliptic operators, Math. Ann., 235 (1978), 55-85.

[13] — On the eigenvalues of a class of hypoelliptic operators II, Global Analysis, Lecture Notes in Math., 755, Springer, 1979, 201-247.

[14] Sjöstrand, J., On the eigenvalues of a class of hypoelliptic operators IV, preprint. 
